\title{
FOCUSING AT A POINT AND ABSORPTION OF NONLINEAR OSCILLATIONS
}

\author{
J. L. JOLY, G. MÉTIVIER, AND J. RAUCH
}

\begin{abstract}
Several recent papers give rigorous justifications of weakly nonlinear geometric optics. All of them consider oscillating wave trains on domains where focusing phenomena do not exist, either because the space dimension is equal to one, or thanks to a coherence assumption on the phases. This paper is devoted to a study of some nonlinear effects of focusing. In a previous paper, the authors have given a variety of examples which show how focusing in nonlinear equations can spoil even local existence in the sense that the domain of existence shrinks to zero as the wavelength decreases to zero. On the other hand, there are many problems for which global existence is known and in those cases it is natural to ask what happens to oscillations as they pass through a focus. The main goal of this paper is to present such a study for some strongly dissipative semilinear wave equations and spherical wavefronts which focus at the origin. We show that the strongly nonlinear phenomenon which is produced is that oscillations are killed by the simultaneous action of focusing and dissipation. Our study relies on the analysis of Young measures and two-scale Young measures associated to sequences of solutions. The main step is to prove that these measures satisfy appropriate transport equations. Then, their variances are shown to satisfy differential inequalities which imply a propagation result for their support.
\end{abstract}

\section{INTRODUCTION}

Weakly nonlinear geometric optics for hyperbolic partial differential equations involve phases which are solutions of eikonal equations as in the linear theory. Recently, there has been much progress on the justification and study of this weakly nonlinear regime; see [JR], [G1, 2], [JMR1,2,3,4], [S]. When the space has dimension at least equal to two, solutions of the eikonal equations exist only locally, the breakdown corresponding to focusing. The justifications of the weakly nonlinear geometric optics, quoted in the papers above, are given on domains where focusing phenomena do not exist, either because the space dimension is equal to one, or thanks to a "coherence" assumption on the phases. It is important to understand better the nonlinear effects of focusing.

When rays focus, amplitudes grow and even in the linear case, one must change the asymptotic description. In the nonlinear case, the large amplitudes

Received by the editors June 14, 1993 and, in revised form, September 13, 1994.

1991 Mathematics Subject Classification. Primary 35B40, 35B05, 35C20, 35L15, 35L70, 35Q72.

Key words and phrases. Oscillation, focusing, dissipation, geometric optics, Young measure, transport equation, independence, absorption, nonlinear waves.

The authors were partially supported by NATO grant CRG 890904; the third author was partially supported by NSF and ONR grants DMS 9203413, N-014-92-J-1245. 
may give rise to strongly nonlinear phenomena. In [JMR 1,2], a variety of examples are given which show how focusing can spoil even local existence in the sense that the domain of existence shrinks to zero as the wavelength decreases to zero. On the other hand, there are many problems for which global existence is known and in those cases it is natural to ask what happens to oscillations as they pass through a focus. The main goal of this paper is to present such a study for some strongly dissipative nonlinearities and spherical wavefronts which focus at the origin. We show that the strongly nonlinear phenomenon which is produced is that oscillations are killed by the simultaneous action of focusing and dissipation. Formal arguments easily yield this conclusion. In this paper, we will make them rigorous in the case of semilinear wave equations and solutions which are smooth with respect to the angular variables. We believe that this phenomenon of nonlinear absorption is much more general. A related phenomenon is rigorously established in [RR2] which analyses one-dimensional problems. The analogue of focusing is that sequences of initial data are studied which concentrate on measure zero sets. The solutions tend to zero in the case of superlinear dissipation. It is entirely plausible that oscillations are absorbed by strongly dissipative nonlinearities and general caustics. The latter problem is beyond our current technique. For weaker nonlinearities, namely those which are uniformly Lipschitzean, the analysis is carried out in [JMR5] and the phenomenon is diametrically opposite. The oscillations survive after focusing. The paper [RR2] also has a dichotomy of behavior depending on whether the nonlinearity is super or sublinear.

Consider a semilinear wave equations in $\mathbb{R}^{1+d}$ of the form

$$
\square u^{\varepsilon}+F\left(t, x, \nabla_{t, x} u^{\varepsilon}\right)=0
$$

where $\square:=\partial_{t}^{2}-\Delta_{x}$ denotes the standard d'Alembertian, and $F(t, x, v)$ is a smooth function of its arguments. Introduce oscillating Cauchy data

$$
u_{\mid t=0}^{\varepsilon}=h(x)+\varepsilon \mathscr{U}_{0}\left(x, \varphi_{0}(x) / \varepsilon\right), \quad \partial_{t} u_{\mid t=0}^{\varepsilon}=\mathscr{U}_{1}\left(x, \varphi_{0}(x) / \varepsilon\right)
$$

where the profiles $\mathscr{U}_{0}(x, \theta)$ and $\mathscr{U}_{1}(x, \theta)$ are periodic in $\theta$. For simplicity, assume that $h, \mathscr{U}_{0}$ and $\mathscr{U}_{1}$ are smooth and have compact support in $\mathbb{R}^{d} \times \mathbb{T}$. The initial phase $\varphi_{0}(x)$ is smooth and $d \varphi_{0} \neq 0$ on the supports of $\mathscr{U}_{0}$ and $\mathscr{U}_{1}$.

Equation (1.1) is a typical example of so-called two-speed equations, studied by Rauch and Reed [RR1]. They proved the existence of "striated" solutions $u^{\varepsilon}$, at least for times $0 \leq t \leq T$, with $T$ possibly small but independent of $\varepsilon$. The asymptotic description of $u^{\varepsilon}$ as the superposition of two wave trains is given by Joly and Rauch [JR],

$$
u^{\varepsilon}(t, x) \sim \underline{u}(t, x)+\varepsilon \mathscr{U}_{+}\left(t, x, \varphi_{+}(t, x) / \varepsilon\right)+\varepsilon \mathscr{U}_{-}\left(t, x, \varphi_{-}(t, x) / \varepsilon\right)
$$

where $\varphi_{ \pm}$are the two solutions of the eikonal equation

$$
\left(\partial_{t} \varphi\right)^{2}=\left|\nabla_{x} \varphi\right|^{2}, \quad \varphi_{\mid t=0}=\varphi_{0} .
$$

Moreover $\underline{u}$ and $\mathscr{U}_{ \pm}$(or more precisely $\partial_{\theta} \mathscr{U}_{ \pm}$) are determined as the solutions of a coupled system of integrodifferential equations which are described below.

The description (1.3) is valid as long as the phases are regular, i.e. as long as the rays do not focus, and as long as the solutions of the profile equations exist. The question we discuss is the long time behaviour of the solutions $u^{\varepsilon}$. To 
understand the phenomena, one can use the following formal argument. For a nonlinearity of the form $F=F\left(\partial_{t} u\right)$ with $F$ odd, and Cauchy data (1.2) with $h=0, \mathscr{U}_{1}=\partial_{\theta} \mathscr{U}_{0}$ odd in $\theta$, and $\varphi_{0}(x)=|x|$, the second wave in (1.3) is not present and

$$
u^{\varepsilon}(t, x) \sim \varepsilon \mathscr{U}_{-}(t, x,(t+|x|) / \varepsilon)
$$

where $\mathscr{V}:=\partial_{\theta} \mathscr{U}_{-}$satisfies

$$
2\left(\partial_{t}-\partial_{r}\right) \mathscr{V}-\frac{d-1}{r} \mathscr{V}+F(\mathscr{V})=0, \quad r:=|x| .
$$

This solution models a spherical wave train which propagates towards $x=0$.

The behaviour of the solutions $\mathscr{V}$ to the o.d.e. (1.6) as $r$ tends to 0 depends on the nonlinearity $F$. Consider the simple example where $F(\lambda)=a|\lambda|^{p-2} \lambda$, where $p \geq 2$. Several possibilities occur.

(A) The linear case, $a=0$ or $p=2$. Then $\mathscr{V}$ behaves like $r^{-(d-1) / 2}$ as $r \rightarrow 0$ on the rays $t+r=$ constant. In spite of this apparent blow up, the solution $u^{\varepsilon}$ exists globally and is smooth. In particular, the local density of energy transported by the oscillations, $r^{(d-1)} \mathscr{V}^{2}$, is bounded as $r$ tends to 0 .

(B) The superlinear dissipative case: $\beta:=(d-1)(p-2) / 2 \geq 1$ and $a>$ 0 . Then the solution does not blow up before $r=0$. There it behaves like $r^{-(d-1) / 2 \beta}$ if $\beta>1$ [resp. $(r|\operatorname{Ln} r|)^{-(d-1) / 2}$ if $\beta=1$ ]. The blow up is therefore weaker than in the linear case, and the local density of energy of oscillations $r^{(d-1)} \mathscr{V}^{2}$ tends to 0 as $r$ tends to 0 . This is the first indication that oscillations are dissipated. In this case, equation (1.1) is dissipative and unique global weak solutions are known to exist (see [LS], [S]). Most of the paper is devoted to this case, and we will explain below that oscillations are absorbed at $r=0$. A related phenomenon of strongly nonlinear dissipation is analyzed in [RR2].

(C) The superlinear accretive case: $\beta:=(d-1)(p-2) / 2 \geq 1$ and $a<0$. Then both terms $-(d-1) \mathscr{V} / r$ and $a F(\mathscr{V})$ contribute to blow up. The two effects add. Because of the first term, $\mathscr{V}$ is necessarily large for small $r$, but then the nonlinear term forces the solution to blow up very quickly. All together, the solution $\mathscr{V}$ of (1.6) explodes before reaching $r=0$, on those rays where the initial condition does not vanish. This indicates that the solutions cannot exist after focusing. The last section of this paper is devoted to this phenomenon.

(D) The weakly nonlinear case: $\beta:=(d-1)(p-2) / 2<1$. When $a \geq 0$, $\mathscr{V} \sim r^{-(d-1) / 2}$ as in the linear case. In this case the equation is dissipative, and global solutions exist. The behaviour of the solution after focusing remains an open problem. In the same category are the equations with sublinear nonlinearities. When $a<0$, the solution may blow up at $r=0$ at the linear rate $r^{-(d-1) / 2}$ or before $r=0$, depending on the size of the initial data.

When more general interactions $F\left(\nabla_{t, x} u\right)$ are involved, the profile equations are more complicated than (1.6) and the discussion is difficult. When dealing with generic caustics, the transport equation, which is justified by [JR] before the caustics, is still of the form (1.6) with $d=2$. Thus it is reasonable to think that phenomena analogous to those above occur in that case too.

The main object of this paper is to study the superlinear dissipative case (B) above. We already observed that the oscillations are dissipated as $r \rightarrow 0$ along the rays. In fact, oscillations are entirely absorbed at $r=0$. To explain this phenomenon, consider the transport equations for the outgoing wave, which 
corresponds to the phase $|x|-t$. It is similar to (1.6):

$$
2\left(\partial_{t}+\partial_{r}\right) \mathscr{W}+\frac{d-1}{r} \mathscr{W}+F(\mathscr{W})=0, \quad r:=|x| .
$$

On $\{t<r\}, \mathscr{W}$ is an outgoing wave generated at $t=0$ from the Cauchy data. On $\{t>r\}$, the ray $t-r=\underline{t}>0$, starting from $(t=\underline{t}, r=0)$, corresponds to the continuation (or reflection) of the focusing ray $t+r=\underline{t}$ for $\mathscr{V}$. Thus, on $\{t>r\}, \mathscr{W}$ is the continuation after focusing of the incoming wave $\mathscr{V}$. The analysis of $(1.7)$ on $\{t>r\}$ is striking. Both terms $(d-1) \mathscr{W} / r$ and $F(\mathscr{W}):=+\mathscr{W}|\mathscr{W}|^{p-2}$ are dissipative. Taking $s=t-\underline{t}$ as a parameter on the ray $t-r=\underline{t}>0,(1.7)$ is the o.d.e.

$$
2 w^{\prime}+\frac{d-1}{s} w+w|w|^{p-2}=0 .
$$

The basic remark is the following.

Lemma 1.1. Assume that $\beta:=(d-1)(p-2) / 2 \geq 1$. Then, for any $\alpha>0$, the only locally $L^{p}$ solution of (1.8) on $] 0, \alpha[$ is $w \equiv 0$.

Proof. First remark that any locally $L^{p}$ solution on $] 0, \alpha\left[\right.$ is $C^{1}$. Introduce $z=s^{(d-1) / 2} w$ so

$$
2 z^{\prime}+s^{-\beta} z|z|^{p-2}=0 .
$$

Assume $\beta>1$. If $z(\sigma) \neq 0$ at some point $\sigma \in] 0, \alpha[$, there is a unique $\left.s_{0} \in\right] 0, \sigma[$ such that

$$
s_{0}^{1-\beta}=\sigma^{1-\beta}+\gamma|z(\sigma)|^{2-p}, \quad \gamma:=2(\beta-1) /(p-2) .
$$

Then the maximal interval of existence of $w$ is $] s_{0},+\infty[$ and on this interval

$$
\gamma|z(s)|^{2-p}=s_{0}^{1-\beta}-s^{1-\beta} \text {. }
$$

This implies that the only solution of (1.9) on $] 0, \alpha[$ is $z=0$. The case $\beta=1$ is similar: $s_{0}$ is the solution of $\operatorname{Ln}\left(\sigma / s_{0}\right)=\gamma|z(\sigma)|^{2-p}, \gamma:=2 /(p-2)$.

This indicates that the only possible $\mathscr{W}$ on the reflected rays (i.e. after focusing) is $\mathscr{W}=0$, which means that no oscillations are present on the reflected ray and that the incoming oscillations have been absorbed at the origin. The discussion above is formal for two reasons. First, interactions between incoming and reflected waves are neglected. Second, it shows that oscillations described by profiles satisfying (1.7) cannot exist beyond the focus. One might have oscillations of a different type. However, it gives a good intuition for the phenomenon. Most of the work in this paper consists in making the argument correct. data

Our approach to the problem is the following. Consider a family of Cauchy

$$
u_{\mid t=0}^{\varepsilon}=u_{0}^{\varepsilon}, \quad \partial_{t} u_{\mid t=0}^{\varepsilon}=u_{1}^{\varepsilon}
$$

with $u_{0}^{\varepsilon}$ bounded in $H^{1}\left(\mathbb{R}^{d}\right)$ and $u_{1}^{\varepsilon}$ bounded in $L^{2}\left(\mathbb{R}^{d}\right)$. With these data, the problem (1.1) with nonlinearity $F=\left|\partial_{t} u\right|^{p-2} \partial_{t} u$ has a global family of weak solutions $u^{\varepsilon}$ bounded in $L^{\infty}\left(\left[0,+\infty\left[; H^{1}\left(\mathbb{R}^{d}\right)\right)\right.\right.$ with $\partial_{t} u^{\varepsilon}$ bounded in $L^{\infty}\left(\left[0,+\infty\left[; L^{2}\left(\mathbb{R}^{d}\right)\right) \cap L^{p}\left(\left[0,+\infty\left[\times \mathbb{R}^{d}\right)\right.\right.\right.\right.$. We introduce Young measures and two-scale Young measures, for the bounded family in $L^{2}, v^{\varepsilon}:=\nabla u^{\varepsilon}$. The main 
step is to prove propagation equations for these measures. These equations imply a propagation inequality for the variances of the measures. A formal derivation of such equations is easy, but correct proofs are more complicated, partly because $v^{\varepsilon}$ need not be bounded in $L^{\infty}$.

Next, propagation inequalities are used to prove that the variances are zero, implying that the corresponding Young measures are Dirac measures. Two distinct reasons are invoked for the vanishing of the variances. The first is propagation from the Cauchy data. The second is a uniqueness principle similar to Lemma 1.1. The first one is applied to two-scale Young measures and implies that if the Cauchy data have oscillating profiles like (1.2) with $\varphi_{0}(x):=\chi(|x|)$, then the solution $u^{\varepsilon}$ also has profiles like (1.3), with $\varphi_{ \pm}(t, x)=\chi(|x| \pm t)$. The second argument is applied to the usual Young measure in the region located after focusing, and the vanishing of the variance implies strong convergence, which means no oscillations.

The paper is organized as follows. The assumptions and main results are stated in section 2 . In section 3 , we prove the compactness theorems after the introduction of Young measures and the derivation of their propagation equations. In section 4, we resume this study with two-scale Young measures and apply the result to the derivation and justification of nonlinear geometric optics approximation. Finally, section 5 contains two examples of nondissipative equations, exhibiting blow up and break down of existence by focusing.

\section{ASSUMPTIONS AND MAIN RESUlTS}

2.1. Global weak solutions. Consider in $\mathbb{R}^{1+d}$ the semilinear Cauchy problem

$$
\square u^{\varepsilon}+F\left(\partial_{t} u^{\varepsilon}\right)=0, \quad u_{\mid t=0}^{\varepsilon}=u_{0}^{\varepsilon}, \quad \partial_{t} u_{\mid t=0}^{\varepsilon}=u_{1}^{\varepsilon}
$$

where $\square:=\partial_{t}^{2}-\Delta_{x}, F(\lambda):=\lambda|\lambda|^{p-2}$ and $p \geq 2$. Our main concern is the superlinear case where

$$
\beta:=(d-1)(p-2) / 2 \geq 1 .
$$

As mentioned in the introduction, more general nonlinearities could be considered, but our analysis relies strongly on the superlinear dissipative behaviour of $F$. For $p \geq 4$, condition (2.1.2) is satisfied in all dimensions $d \geq 2$. In dimension $d=3$, condition (2.1.2) is $p \geq 3$.

The Cauchy data are assumed to satisfy the following conditions.

Assumption 2.1.1. The families $u_{0}^{\varepsilon}$ and $u_{1}^{\varepsilon}$ are bounded in $H^{1}\left(\mathbb{R}^{d}\right)$ and $L^{2}\left(\mathbb{R}^{d}\right)$ respectively. Moreover they are angularly smooth; that is, for all $k$ and $l$, $\Gamma_{k, l} u_{0}^{\varepsilon}$ is bounded in $H^{1}\left(\mathbb{R}^{d}\right)$ and $\Gamma_{k, l} u_{1}^{\varepsilon}$ is bounded in $L^{2}\left(\mathbb{R}^{d}\right)$, where $\Gamma_{k, l}$ denotes the vector field

$$
\Gamma_{k, l}:=x_{l} \partial_{x_{k}}-x_{k} \partial_{x_{l}}
$$

An example is data which have radial oscillations, as in (1.2). A precise study of this special case is presented in subsection 2.4 .

Under assumption 2.1.1, one has existence of global solutions for the Cauchy problem (2.1.1).

Theorem 2.1.2. The Cauchy problem (2.1.1) has a unique solution $u^{\varepsilon}$ in $C^{0}\left(\left[0,+\infty\left[; H^{1}\left(\mathbb{R}^{d}\right)\right)\right.\right.$ with $\partial_{t} u^{\varepsilon} \in C^{0}\left(\left[0,+\infty\left[; L^{2}\left(\mathbb{R}^{d}\right)\right) \cap L^{p}\left(\left[0,+\infty\left[\times \mathbb{R}^{d}\right)\right.\right.\right.\right.$. 
Moreover for any $(k, l), \Gamma_{k, l} u^{\varepsilon}$ belongs to $C^{0}\left(\left[0,+\infty\left[; H^{1}\left(\mathbb{R}^{d}\right)\right)\right.\right.$. Finally, one has the following uniform estimates:

$$
\begin{gathered}
\left\|\nabla_{t, x} u^{\varepsilon}(t)\right\|_{L^{2}\left(\mathbb{R}^{d}\right)}^{2}+2 \int_{0}^{t}\left\|\partial_{t} u^{\varepsilon}\left(t^{\prime}\right)\right\|_{L^{p}\left(\mathbb{R}^{d}\right)}^{p} d t^{\prime}=\left\|\nabla_{t, x} u^{\varepsilon}(0)\right\|_{L^{2}\left(\mathbb{R}^{d}\right)}^{2}, \\
\left\|\nabla_{t, x} \Gamma_{k, l} u^{\varepsilon}(t)\right\|_{L^{2}\left(\mathbb{R}^{d}\right)}^{2} \leq C .
\end{gathered}
$$

Proof. The solutions are constructed in the works [L] and [LS]. They belong to $C^{0}\left(\left[0,+\infty\left[; H^{1}\left(\mathbb{R}^{d}\right)\right)\right.\right.$ and $\partial_{t} u^{\varepsilon} \in C^{0}\left(\left[0,+\infty\left[; L^{2}\left(\mathbb{R}^{d}\right)\right) \cap L^{p}\left(\left[0,+\infty\left[\times \mathbb{R}^{d}\right)\right.\right.\right.\right.$. Moreover $t \rightarrow u^{\varepsilon}(t)$ and $t \rightarrow \partial_{t} u(t)$ are continuous with values in $H^{1}$ and $L^{2}$ respectively. They satisfy (2.1.4) which is the standard energy estimate.

Let $R_{h}$ be a one-parameter group of rotations in $\mathbb{R}^{d}$. $\square$ commutes with $R_{h}$ so that $v:=R_{h} u-u$ satisfies

$$
\square v+G\left(\partial_{t} R_{h} u, \partial_{t} u\right) \partial_{t} v=0,
$$

where $G(\lambda, \mu):=\{F(\lambda)-F(\mu)\} /(\lambda-\mu) . G\left(\partial_{t} R_{h} u, \partial_{t} u\right) \partial_{t} v$ belongs to $L^{p^{\prime}}$, with $p^{\prime}$ the conjugate exponent of $p$. So $G\left(\partial_{t} R_{h}, \partial_{t} u\right)\left(\partial_{t} v\right)^{2}$ is integrable and $\geq 0$. Thus, multiplying (2.1.6) by $\partial_{t} v$ and integrating by parts yield formally

$$
\left\|\nabla_{t, x} v(t)\right\|_{L^{2}\left(\mathbb{R}^{d}\right)}^{2} \leq\left\|\nabla_{t, x} v(0)\right\|_{L^{2}\left(\mathbb{R}^{d}\right)}^{2} .
$$

Divide (2.1.7) by $h$ and let $h$ tend to 0. This yields (2.1.5).

2.2. Statement of the problem. We study the weak limits of the family $u^{\varepsilon}$. Let $\Omega$ denote the open set $] 0,+\infty\left[\times \mathbb{R}^{d}\right.$, and $\left.\Omega_{T}:=\right] 0, T\left[\times \mathbb{R}^{d}\right.$. Estimates (2.1.4), (2.1.5) imply that one can extract subsequences, still called $u^{\varepsilon}$, such that

$$
u^{\varepsilon} \rightarrow \underline{u} \text { weakly in } H^{1}\left(\Omega_{T}\right) \text { and strongly in } L_{\text {loc }}^{2}(\bar{\Omega}),
$$

(2.2.2) $\quad \Gamma_{k, l} u^{\varepsilon} \rightarrow \Gamma_{k, l} \underline{u}$ weakly in $H^{1}\left(\Omega_{T}\right)$ and strongly in $L_{\text {loc }}^{2}(\bar{\Omega})$,

$$
F\left(\partial_{t} u^{\varepsilon}\right) \rightarrow \underline{F} \text { weakly in } L^{p}(\Omega) .
$$

One can also assume that the Cauchy data have limits as $\varepsilon$ tends to 0 .

$$
\begin{gathered}
u_{0}^{\varepsilon} \rightarrow \underline{u}_{0} \text { weakly in } H^{1}\left(\mathbb{R}^{d}\right) \text { and strongly in } L_{\text {loc }}^{2}\left(\mathbb{R}^{d}\right), \\
u_{1}^{\varepsilon} \rightarrow \underline{u}_{1} \text { weakly in } L^{2}\left(\mathbb{R}^{d}\right) .
\end{gathered}
$$

Then, $\underline{u}$ is the solution of the Cauchy problem

$$
\begin{gathered}
\square \underline{u}+\underline{F}=0, \\
\underline{u}_{\mid t=0}=\underline{u}_{0}, \quad \partial_{t} \underline{u}_{\mid t=0}=\underline{u}_{1} .
\end{gathered}
$$

In this context, the main question is to identify the limit $\underline{F}$, which is not necessarily equal to $F\left(\partial_{t} \underline{u}\right)$ since $\partial_{t} u^{\varepsilon}$ need not be strongly convergent. However, (2.2.2) implies that the $\Gamma_{k, l} u^{\varepsilon}$ are compact, so any lack of compactness in $\nabla_{t, x} u^{\varepsilon}$ lies entirely in the time derivative $\partial_{t} u^{\varepsilon}$ and the radial derivative $\partial_{r} u^{\varepsilon}$, where

$$
\partial_{r}:=\frac{1}{r} \sum_{j=1}^{d} x_{j} \partial_{x_{j}} .
$$

Polar coordinates on $\mathbb{R}^{d} \backslash\{0\}$ are denoted

$$
r:=|x|, \quad \hat{x}:=x /|x| \in S^{d-1} \text {. }
$$


Let $\left.\Omega^{*}:=\right] 0,+\infty\left[\times \mathbb{R}^{d} \backslash\{0\}\right.$. Introduce

$$
v_{ \pm}^{\varepsilon}:=\frac{1}{2}\left(\partial_{t} \mp \partial_{r}\right) u^{\varepsilon}
$$

which are bounded families in $C^{0}\left(\left[0,+\infty\left[; L^{2}\left(\mathbb{R}^{d}\right)\right)\right.\right.$. On $\Omega^{*}$

$$
\Delta_{x}=\partial_{r}^{2}+(d-1) r^{-1} \partial_{r}+r^{-2} \Delta_{\hat{x}}, \quad \text { where } \Delta_{\hat{x}}:=\sum_{j<k} \Gamma_{j, k}^{2}
$$

and $v_{ \pm}^{\varepsilon}$ satisfy

$$
\begin{gathered}
2\left(\partial_{t} \pm \partial_{r}\right) v_{ \pm}^{\varepsilon}+(d-1) r^{-1}\left(v_{+}^{\varepsilon}-v_{-}^{\varepsilon}\right)-r^{-2} \Delta_{\hat{x}} u^{\varepsilon}+F\left(v_{+}^{\varepsilon}+v_{-}^{\varepsilon}\right)=0, \\
v_{ \pm \mid t=0}^{\varepsilon}=v_{0, \pm}^{\varepsilon}:=\frac{1}{2}\left(u_{1}^{\varepsilon} \mp \partial_{r} u_{0}^{\varepsilon}\right) .
\end{gathered}
$$

Our program is to study the behaviour of $v_{ \pm}^{\varepsilon}$ and of $F\left(v_{+}^{\varepsilon}+v_{-}^{\varepsilon}\right)$ as $\varepsilon \rightarrow 0$.

2.3. Propagation of compactness and absorption of oscillations. We first introduce a general definition.

Definition 2.3.1. A bounded family $z^{\varepsilon}(y)$ in $L^{q}(\mathscr{O})$ is compact at $y_{0} \in \mathscr{O}$ if there is a neighborhood $\omega \subset \mathscr{O}$ of $y_{0}$ such that the restrictions of $z^{\varepsilon}$ to $\omega$ lie in a compact subset of $L^{q}(\omega)$.

Assumptions and notations are those of subsections 1 and 2. A first result proves propagation of compactness for $v_{ \pm}^{\varepsilon}$ in $|x|>0$, along the rays $x=$ $x_{0} \pm t \hat{x}_{0}$, where $\hat{x}_{0}:=x_{0} /\left|x_{0}\right|$.

Theorem 2.3.2. (i) Assume that the Cauchy data $v_{0,+}^{\varepsilon}$ is compact in $L^{2}$ at a point $x_{0} \neq 0$. Then $v_{+}^{\varepsilon}$ is compact in $L^{2}$ at points $(t, x)$ such that $t>0$, $x=x_{0}+t \hat{x}_{0}$.

(ii) Assume that the Cauchy data $v_{0,-}^{\varepsilon}$ is compact in $L^{2}$ at a point $x_{0} \neq 0$. Then $v_{-}^{\varepsilon}$ is compact in $L^{2}$ at points $(t, x)$ such that $0<t<\left|x_{0}\right|$ and $x=$ $x_{0}-t \hat{x}_{0}$.

This result states that compactness is propagated from the Cauchy data along bicharacteristics, as long as they do not cross the origin $\{x=0\} . v_{+}^{\varepsilon}$ is an expansive wave and $v_{-}^{\varepsilon}$ is compressive. Reversing time, this theorem states that $v_{-}^{\varepsilon}$ is compact at $(t, x) \in \Omega^{*}$, whenever its Cauchy data is compact at $x_{0}=x+t \hat{x}$. Similarly, $v_{+}^{\varepsilon}$ is compact at a point $(t, x) \in \Omega^{*}$ located outside the light cone issued from the origin, i.e. such that $t<|x|$, if its Cauchy data is compact at $x_{0}=x-t \hat{x}$.

It remains to describe $v_{+}^{\varepsilon}$ inside the light cone $\mathscr{C}:=\{(t, x)|t>| x \mid\}$. There $v_{+}^{\varepsilon}$ represents an outgoing wave which is the continuation of a wave which has focused on the axis $\mathscr{A}:=\{x=0\}$. We have an absorption phenomenon which is one of the main points of this paper.

Theorem 2.3.3. Assume condition (2.1.2). Then $v_{+}^{\varepsilon}$ is compact in $L^{2}$ at points $(t, x) \in \mathscr{C} \backslash \mathscr{A}$, that is, points in the solid light cone $\mathscr{C}$ with the axis removed. At points of the axis $\mathscr{A}, v_{+}^{\varepsilon}$ is compact in $L^{q}$ for all $q<2$.

For the original $u^{\varepsilon}$, this implies 
Theorem 2.3.4. Assume condition (2.1.2). Let $x_{ \pm}:=x \mp t x /|x|$ denote the feet of the two radial characteristics passing through $(t, x) \in \Omega^{*}$. Then:

(i) If $(t, x) \notin \mathscr{C}, \nabla_{t, x} u^{\varepsilon}$ is compact in $L^{2}$ at $(t, x)$ as soon as $u_{1}^{\varepsilon}$ and $\nabla_{x} u_{0}^{\varepsilon}$ are compact in $L^{2}$ at $x_{+}$and $x_{-}$.

(ii) For $(t, x) \in \mathscr{C} \backslash \mathscr{A}, \nabla_{t, x} u^{\varepsilon}$ is compact in $L^{2}$ at $(t, x)$ if $u_{1}^{\varepsilon}$ and $\nabla_{x} u_{0}^{\varepsilon}$ are compact in $L^{2}$ at $x_{-}$.

(iii) If. $u_{1}^{\varepsilon}$ and $\nabla_{x} u_{0}^{\varepsilon}$ are compact in $L^{2}$ at points of the sphere $\left\{|x|=t_{0}\right\}$, then $\nabla_{t, x} u^{\varepsilon}$ is compact in $L^{q}$ at $\left(t_{0}, 0\right) \in \mathscr{A}$, for all $q<2$.

Remark 2.3.5. The two theorems do not assert compactness in $L^{2}$ at points of the axis $\mathscr{A}$ and thus do not exclude concentration effects along the time axis. We do not know whether this possibility can actually occur. A more refined analysis, in particular of the boundary condition for $v_{+}^{\varepsilon}$ on $r=0$, would be necessary to eliminate this ambiguity. Such an analysis would also be needed to study the case $\beta<1$.

Example 2.3.6. Suppose that the initial data $\left(u_{0}^{\varepsilon}, u_{1}^{\varepsilon}\right)$ are supported in $1 \leq$ $|x| \leq 2$. Then, assuming that $(p-2)(d-1) \geq 2, \nabla_{t, x} u^{\varepsilon}$ is compact in the domain $\mathscr{D}:=\{t \geq \sup (2-|x|,|x|-1)\}$, sketched in Figure 1 .

Note that in the linear case, oscillations and noncompactness are propagated in the domain $\{|x|+1<t<|x|+2\} \subset \mathscr{D}$. This domain is bounded by the dotted lines in Figure 1. Thus the nonlinear effect of absorption leads to a truly different picture.

If the Cauchy data $v_{0,+}^{\varepsilon}$ converge strongly in $L^{2}$, then $v_{+}^{\varepsilon}$ is compact everywhere and $\nabla_{t, x} u^{\varepsilon}$ is compact on $\{t>2-|x|\} \supset\{t>2\}$. This implies that, after taking subsequences, $\nabla u^{\varepsilon}$ converges strongly in $L_{\text {loc }}^{q}$ for $t \geq 2$ and that the limit $\underline{F}$ in $(2.2 .3)$ is $F\left(\partial_{t} \underline{u}\right)$. Thus, for $t>2, \underline{u}$ satisfies

$$
\square \underline{u}+F\left(\partial_{t} \underline{u}\right)=0 .
$$

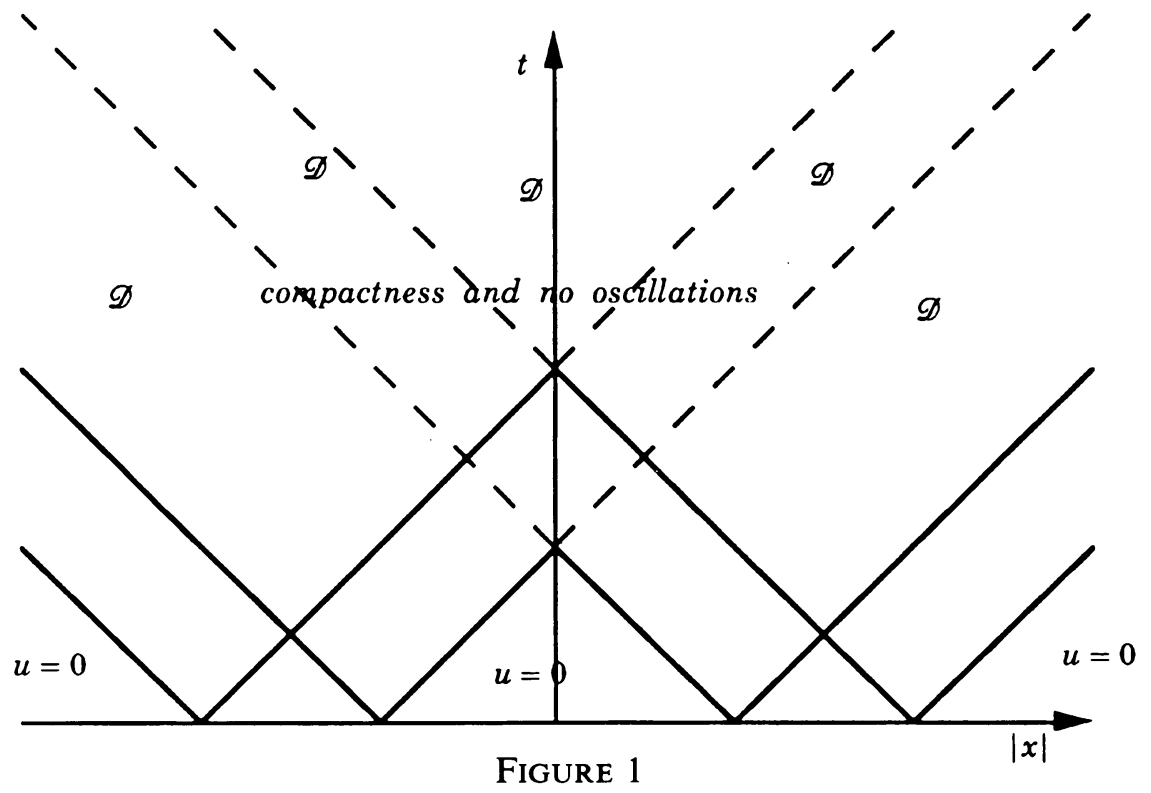


To determine $\underline{u}$ for $t>2$, it is therefore sufficient to know the Cauchy data of $\underline{u}$ at time $t=2$. We stress the fact that in general $\underline{F} \neq F\left(\partial_{t} \underline{u}\right)$ in $\{2-|x| \geq t \geq 1-|x|\}$, so that the values of $\nabla \underline{u}$ at $t=2$ are not determined by $\left(\underline{u}_{0}, \underline{u}_{1}\right)$. The next paragraph explains how to compute $\underline{F}$ and $\underline{u}$ in the case of oscillating data.

2.4. Geometric optics. Consider the special case of Cauchy data which are high frequency oscillating wave trains.

Assumption 2.4.1. $u_{0}^{\varepsilon}$ and $u_{1}^{\varepsilon}$ are of the form

$$
\begin{gathered}
u_{0}^{\varepsilon}(x)=\underline{u}_{0}(x)+\varepsilon \mathscr{U}_{0}(x, \chi(|x|) / \varepsilon)+r_{0}^{\varepsilon}, \\
u_{1}^{\varepsilon}(x)=\mathscr{U}_{1}(x, \chi(|x|) / \varepsilon)+r_{1}^{\varepsilon}
\end{gathered}
$$

where $r_{0}^{\varepsilon}$ and $r_{1}^{\varepsilon}$ converge strongly to 0 , respectively in $H^{1}\left(\mathbb{R}^{d}\right)$ and in $L^{2}\left(\mathbb{R}^{d}\right)$. In addition we suppose that for any $k$ and any $l, \Gamma_{k, l} r_{0}^{\varepsilon}$ and $\Gamma_{k, l} r_{1}^{\varepsilon}$ converge strongly to 0 in $H^{1}\left(\mathbb{R}^{d}\right)$ and $L^{2}\left(\mathbb{R}^{d}\right)$ respectively.

The function $\chi$ is smooth with $\chi^{\prime} \neq 0$ a.e. and $\chi^{\prime} \in L^{\infty}(\mathbb{R})$. The profiles $\mathscr{U}_{0}(x, \theta)$ and $\mathscr{U}_{1}(x, \theta)$ are smooth in $(x, \theta)$, periodic in $\theta$, bounded with bounded derivatives on $\mathbb{R}^{d} \times \mathbb{T}$.

Note that such $u_{0}^{\varepsilon}$ and $u_{1}^{\varepsilon}$ satisfy assumption 2.1.1. For simplicity, we assume that the period of the profiles is 1 . Then the fast variables $\theta$ belong to $\mathbb{T}:=\mathbb{R} / \mathbb{Z}$. In fact, very low smoothness is required for the profiles. This point is discussed in section 4 .

The description of the solution $u^{\varepsilon}$ for small time follows [JR]. Provided that the support of the data does not meet $x=0$, one has

$$
u^{\varepsilon}(t, x)=\underline{u}(t, x)+\varepsilon \mathscr{U}_{+}\left(t, x, \varphi_{+}(t, x) / \varepsilon\right)+\varepsilon \mathscr{U}_{-}\left(t, x, \varphi_{-}(t, x) / \varepsilon\right)+o(\varepsilon)
$$

where $\varphi_{ \pm}$are the two solutions of the eikonal equation with data $\chi(|x|)$ :

$$
\varphi_{ \pm}(t, x):=\chi(|x| \mp t) .
$$

Thus, no oscillations of magnitude $O(1)$ are created in the $\Gamma_{k, l} u^{\varepsilon}$, which is consistent with the results of the previous subsection. On the other hand, oscillations are expected in $v_{ \pm}^{\varepsilon}$.

$$
v_{ \pm}^{\varepsilon}:=\frac{1}{2}\left(\partial_{t} \mp \partial_{r}\right) \underline{u}+\mathscr{W}_{ \pm}\left(t, x, \varphi_{ \pm}(t, x) / \varepsilon\right)+o(1)
$$

where

$$
\mathscr{W}_{ \pm}(t, x, \theta):=\mp \chi^{\prime}(|x| \mp t) \partial_{\theta} \mathscr{U}_{ \pm}(t, x, \theta) .
$$

The profiles $\mathscr{U}_{ \pm}$are determined from the following equations for $\left(\underline{u}, \mathscr{W}_{+}, \mathscr{W}_{-}\right)$:

$$
\begin{cases}\square \underline{u}+\underline{F}=0 & \text { on } \Omega, \\ X_{ \pm} \mathscr{W}_{ \pm}+F_{ \pm}\left(t, x, \mathscr{W}_{ \pm}\right)=0 & \text { on } \Omega^{*},\end{cases}
$$

where the transport operators $X_{ \pm}$are those of the linear theory

$$
X_{ \pm} a:=2\left(\partial_{t} \pm \partial_{r}\right) a \pm(d-1) r^{-1} a .
$$


The nonlinear interactions $\underline{F}$ and $F_{ \pm}$involve integrations with respect to fast variables $\theta_{j} \in \mathbb{T}:=\mathbb{R} / \mathbb{Z}$ :

$$
\begin{aligned}
\underline{F}(t, x) & :=\iint \widetilde{F}\left(t, x, \mathscr{W}_{+}\left(t, x, \theta_{1}\right)+\mathscr{W}_{-}\left(t, x, \theta_{2}\right)\right) d \theta_{1} d \theta_{2}, \\
F_{+}(t, x, \lambda) & :=\int \widetilde{F}\left(t, x, \lambda+\mathscr{W}_{-}\left(t, x, \theta_{2}\right)\right) d \theta_{2}-\underline{F}(t, x), \\
F_{-}(t, x, \lambda) & :=\int \widetilde{F}\left(t, x, \mathscr{W}_{+}\left(t, x, \theta_{1}\right)+\lambda\right) d \theta_{1}-\underline{F}(t, x)
\end{aligned}
$$

with

$$
\widetilde{F}(t, x, \lambda):=F\left(\partial_{t} \underline{u}(t, x)+\lambda\right) .
$$

In formulas (2.4.9)-(2.4.11), the integrations are performed on $\mathbb{T}$, with respect to the invariant measure $d \theta$ of total mass 1 .

The Cauchy data for $\underline{u}$ are given by (2.2.7). The weak limit of $u_{0}^{\varepsilon}$ is $\underline{u}_{0}$. We will show later on that the weak limit of $u_{1}^{\varepsilon}$ is the average of $\mathscr{U}_{1}$. This yields

$$
\underline{u}_{\mid t=0}=\underline{u}_{0}, \quad \partial_{t} \underline{u}_{\mid t=0}=\underline{u}_{1}:=\int \mathscr{U}_{1}(\cdot, \theta) d \theta .
$$

The Cauchy data for $\mathscr{W}_{ \pm}$are

$$
\mathscr{W}_{ \pm \mid t=0}(x, \theta)=\frac{1}{2}\left\{\mathscr{U}_{1}^{\text {osc }}(x, \theta) \mp \chi^{\prime}(|x|) \partial_{\theta} \mathscr{U}_{0}(x, \theta)\right\}
$$

where $\mathscr{U}_{1}^{\text {osc }}(x, \theta):=\mathscr{U}_{1}(x, \theta)-\int \mathscr{U}_{1}\left(x, \theta^{\prime}\right) d \theta^{\prime}$.

Our goal is to justify the asymptotic description (2.4.5), for all time, even after focusing. Note that if such an expansion is valid, then, by Theorem 2.3.3, the profile $\mathscr{W}_{+}$must vanish inside the light cone $\mathscr{C}=\{t>|x|\}$.

Theorem 2.4.2. Assume condition (2.1.2). There is a unique solution ( $\left.\underline{u}, \mathscr{W}_{ \pm}\right)$ of (2.4.7) with the Cauchy data (2.4.13), (2.4.14) and satisfying the following properties:

(i) $\underline{u} \in L^{\infty}\left(\left[0, \infty\left[; H^{1}\left(\mathbb{R}^{d}\right)\right)\right.\right.$ and $\partial_{t} \underline{u} \in L^{p}(\Omega) \cap L^{\infty}\left(\left[0,+\infty\left[; L^{2}\left(\mathbb{R}^{d}\right)\right)\right.\right.$,

(ii) $\mathscr{W}_{ \pm} \in L^{\infty}\left(\left[0,+\infty\left[; L^{2}\left(\mathbb{R}^{d} \times T\right)\right) \cap L^{p}(\Omega \times T)\right.\right.$,

(iii) $\partial_{\theta} \mathscr{W}_{ \pm} \in L^{\infty}\left(\left[0,+\infty\left[; L^{2}\left(\mathbb{R}^{d} \times T\right)\right)\right.\right.$,

(iv) $\mathscr{W}_{+}$vanishes inside the solid light cone $c:=\{t>|x|\}$,

(v) for almost all $(t, x) \in\left[0,+\infty\left[\times \mathbb{R}^{d}, \int \mathscr{W}_{ \pm}(t, x, \theta) d \theta=0\right.\right.$.

These $\mathscr{W}_{ \pm}$give a complete description of the oscillations of amplitude $O(1)$ of $\nabla_{t, x} u^{\varepsilon}$.

Theorem 2.4.3. Assume condition (2.1.2). The solutions $u^{\varepsilon}$ satisfy

$$
\begin{gathered}
\partial_{t} u^{\varepsilon}(t, x)=\partial_{t} \underline{u}(t, x)+\mathscr{W}_{+}\left(t, x, \varphi_{+}(t, x) / \varepsilon\right) \\
+\mathscr{W}_{-}\left(t, x, \varphi_{-}(t, x) / \varepsilon\right)+e_{0}^{\varepsilon}(t, x), \\
\partial_{x_{j}} u^{\varepsilon}(t, x)=\partial_{x_{j}} \underline{u}(t, x)+\frac{x_{j}}{|x|}\left\{\mathscr{W}_{-}\left(t, x, \varphi_{-}(t, x) / \varepsilon\right)\right. \\
\left.-\mathscr{W}_{+}\left(t, x, \varphi_{+}(t, x) / \varepsilon\right)\right\}+e_{j}^{\varepsilon}(t, x),
\end{gathered}
$$


where the $e_{j}^{\varepsilon}$ converge strongly in $L^{q}(\omega)$ for all $q<2$ and for all bounded $\omega \subset \Omega$, and in $L^{2}(\omega)$ for all $\omega \subset \subset \Omega^{*}$.

Remark 2.4.4. Because of (ii) and (iii) in Theorem 2.4.2, $\sigma \rightarrow \mathscr{Z}_{ \pm}(\cdot, \cdot, \sigma):=$ $\mathscr{W}_{ \pm}\left(\cdot, \cdot, \sigma+\varphi_{ \pm}(\cdot, \cdot) / \varepsilon\right)$ belongs to $L^{2}\left(\Omega_{T}\right)$ and $\partial_{\theta} \mathscr{Z}_{ \pm} \in L^{2}\left(\Omega_{T}\right)$. Thus $\mathscr{Z}_{ \pm}$ is continuous from $\mathbb{T}$ to $L^{2}\left([0, T] \times \mathbb{R}^{m}\right)$ and the substitution $\theta=\varphi_{ \pm}(t, x) / \varepsilon$ in (2.4.15), (2.4.16) is well defined, as the trace on $\sigma=0$ of $\mathscr{Z}_{ \pm}$. Since the mean values of $\mathscr{W}_{ \pm}$and $\mathscr{Z}_{ \pm}$vanish, one has

$$
\mathscr{Z}_{ \pm}(y, 0)=\int S(\theta) \partial_{\theta} \mathscr{Z}_{ \pm}(y, \theta) d \theta
$$

where $S$ is the periodic saw tooth function such that $-\partial_{\theta} S$ is the Dirac mass at $\theta=0$. This yields

$$
\mathscr{W}_{ \pm}\left(y, \varphi_{ \pm}(y) / \varepsilon\right)=\int S\left(\theta-\varphi_{ \pm}(y) / \varepsilon\right) \partial_{\theta} \mathscr{W}_{ \pm}(y, \theta) d \theta
$$

\section{Proof of THE COMPaCtNess Results}

The proof of the compactness theorems described in section 2.3 relies on an analysis of the Young measures associated to $v_{ \pm}^{\varepsilon}$. After recalling the definition in section 3.1, we prove in section 3.2 that the Young measure of the pair $\left(v_{+}^{\varepsilon}, v_{-}^{\varepsilon}\right)$ is the tensor product of the measures associated to $v_{+}^{\varepsilon}$ and $v_{-}^{\varepsilon}$. This expresses independence or absence of resonance, between the two waves. In subsection 3.3 we derive transport equations for the Young measures and transport inequalities for their variance. The compactness theorems are proved in section 3.4 as corollaries of these inequalities.

3.1. Young measures. Young measures for bounded sequences $u^{\varepsilon} \in L^{\infty}(\Omega)$ are introduced by Young [Y]. This notion was used to prove existence of weak solutions for conservation laws; see Tartar [T], Di Perna [DP1]. It is also related to the notion of measure-valued solution [DP2]. The definition was then extended to sequences in $L^{p}$ [DP-M]. Here we introduce a slightly weaker definition which corresponds to the part of the Young measure of [DP-M] located at finite distance $|\lambda|<+\infty$. It does not take into account the defect measure located at $|\lambda|=\infty$.

In this section $\mathscr{O}$ denotes an open subset of a Euclidean space $\mathbb{R}^{n}$, whose boundary has zero Lebesgue measure. Typically $\mathscr{O}$ will be $] 0,+\infty\left[\times \mathbb{R}^{d}\right.$, ]0, $T\left[\times \mathbb{R}^{d}\right.$ or $\mathbb{R}^{d}$. The starting point is the following.

Proposition 3.1.1. Given a bounded family $\left\{u^{\varepsilon}\right\} \subset L^{p}(\mathscr{O})$ of $\mathbb{R}^{N}$-valued functions, there exists a subsequence and a measurable family of probability measures on $\mathbb{R}^{N},\{\mu(y, \cdot), y \in \mathscr{O}\}$, such that for all continuous functions $f(\lambda)$ on $\mathbb{R}^{N}$ which tend to 0 at infinity and for all $\varphi \in C_{o}^{0}(\mathscr{O})$

$$
\int \varphi(y) f\left(u^{\varepsilon}(y)\right) d y \rightarrow \iint \varphi(y) f(\lambda) \mu(y, d \lambda) d y .
$$

Property (3.1.1) means that

$$
\int \Phi\left(y, u^{\varepsilon}(y)\right) d y \rightarrow \iint \Phi(y, \lambda) \mu(y, d \lambda) d y
$$

when $\Phi(y, \lambda)$ is a product $\varphi(y) f(\lambda)$. By linear combination and density, formula (3.1.2) extends to all $\Phi \in C_{o}^{0}\left(\mathscr{O} \times \mathbb{R}^{N}\right)$. 
Formula (3.1.2) defines a measure $\mu$ on $\mathscr{O} \times \mathbb{R}^{N}$, which we call the Young measure of the subsequence or subfamily. To simplify matters, we introduce the following definition.

Definition 3.1.2. A bounded family $u^{\varepsilon}$ in $L^{p}$ is "pure" when no extraction of subsequences is necessary, i.e. when there exists a measure $\mu$ satisfying (3.1.1).

Note that such a measure is necessarily unique. Using this vocabulary, Proposition 3.1 .1 states that from any bounded family $u^{\varepsilon}$ one can extract a pure subsequence.

(3.1.1) means that $f\left(u^{\varepsilon}\right)$ converges weakly to $\int f(\lambda) \mu(y, d \lambda) \in L_{\text {loc }}^{1}(\mathscr{O})$. This convergence can be extended to functions $f$ such that $f(\lambda)=o\left(|\lambda|^{p}\right)$ at infinity. See below for a precise statement. One could also consider the weak limits of $f\left(u^{\varepsilon}\right)$ when $f(\lambda)=O\left(|\lambda|^{p}\right)$, but these limits would not belong in general to $L_{\text {loc }}^{1}$. This possibility is explored in [DP-M], and it amounts to adding a measure on $\mathscr{O} \times S, S$ the sphere at infinity, to the measure $\mu$ on $\mathscr{O} \times \mathbb{R}^{N}$. The reason why we drop this "concentration" measure is that in general it is not absolutely continuous in $y$ with respect to the Lebesgue measure $d y$, a property which is repeatedly used for $\mu$. For a proof of Proposition 3.1.1, we refer to [T], [DP-M], [Ev].

Proposition 3.1.3. Let $u^{\varepsilon}$ be a pure bounded family in $L^{p}(\mathscr{O})$ with Young measure $\mu$. Then $|\lambda| \in L^{p}\left(\mathscr{O} \times \mathbb{R}^{N}, \mu\right)$ and

$$
\iint|\lambda|^{p} \mu(y, d \lambda) d y \leq \liminf \left\|u^{\varepsilon}\right\|_{L^{p}(\mathscr{O})}^{p}
$$

Proof. Let $\zeta(\lambda)$ be a cut-off function, equal to one on the unit ball, and such that $0 \leq \zeta \leq 1$. Let $\varphi \in C_{o}^{0}(\mathscr{O}), \varphi \geq 0$. Then Fatou's lemma yields

$$
\begin{gathered}
\iint \varphi(y) \zeta(\lambda / k)|\lambda|^{p} \mu(y, d \lambda) d y=\lim \int \varphi(y) \zeta\left(u^{\varepsilon}(y) / k\right)\left|u^{\varepsilon}(y)\right|^{p} d y \\
\leq \liminf \int \varphi(y)\left|u^{\varepsilon}(y)\right|^{p} d y \leq \liminf \left\|u^{\varepsilon}\right\|_{L^{p}(\mathscr{O})}^{p}
\end{gathered}
$$

Fatou's lemma again implies that

$$
\begin{aligned}
& \iint \varphi(y)|\lambda|^{p} \mu(y, d \lambda) d y \\
& \quad \leq \liminf \iint \varphi(y) \zeta(\lambda / k)|\lambda|^{p} \mu(y, d \lambda) d y \leq \liminf \left\|u^{\varepsilon}\right\|_{L^{p}(\mathscr{O})}^{p} .
\end{aligned}
$$

Proposition 3.1.4. Suppose that $u^{\varepsilon}$ is a pure bounded family in $L^{p}(\mathscr{O})$ with Young measure $\mu$ and $F(\lambda, \tau)$ is a continuous function such that $|F(\lambda, \tau)|=$ $o\left(|\lambda|^{p}+|\tau|^{p}\right)$ as $|\lambda|+|\tau| \rightarrow+\infty$. If $a \in L^{p}(\mathscr{O})$ and $\varphi \in C_{o}^{0}(\overline{\mathscr{O}})$, then

$$
\int \varphi(y) F\left(u^{\varepsilon}(y), a(y)\right) d y \rightarrow \iint \varphi(y) F(\lambda, a(y)) \mu(y, d \lambda) d y .
$$

Moreover, if $|F(\lambda, \tau)|=O\left(|\lambda|^{q}+|\tau|^{q}\right)$ as $|\lambda|+|\tau| \rightarrow+\infty$, with $q<p$, then the convergence (3.1.4) extends to $\varphi \in L_{\text {comp }}^{r}(\overline{\mathscr{O}})$, with $1 / r+q / p=1$.

Note that $\varphi(y) F(\lambda, a(y))$ is not necessarily continuous. However it is measurable, defined a.e. on $\mathscr{O} \times \mathbb{R}^{N}$ and integrable with respect to the measure $\mu=\mu(y, d \lambda) d y$. Thus the right-hand side of (3.1.4) makes sense. 
In the statement above, $C_{o}^{0}(\overline{\mathscr{O}})$ denotes the space of continuous functions on the closed set $\overline{\mathscr{O}}$, with compact support. Similarly, $L_{\text {comp }}^{r}(\overline{\mathscr{O}})$ denotes the space of those $\varphi \in L^{r}(\mathscr{O})$ vanishing outside a compact subset of $\overline{\mathscr{O}}$.

Proof. When $a$ is continuous, $F \in C_{o}^{0}$, and $\varphi \in C_{o}^{0}(\mathscr{O})$, (3.1.4) follows from (3.1.2) applied to $\Phi(y, \lambda)=\varphi(y) F(\lambda, a(y))$.

(a) Because the boundary $\partial \mathscr{O}$ is negligible, the convergence (3.1.4) extends to the case where $\varphi \in C_{o}^{0}(\overline{\mathscr{O}}), F \in C_{o}^{0}$ and $a \in C^{0}$, since $F$ is bounded in that case.

(b) Suppose next that $\varphi \in C_{o}^{0}(\overline{\mathscr{O}}), F \in C_{o}^{1}$ and $a \in L^{1}(\mathscr{O})$. Approximate $a$ in $L^{1}(\mathscr{O})$ by continuous functions $a_{k}$. On one side $\varphi F\left(u^{\varepsilon}, a_{k}\right) \rightarrow \varphi F\left(u^{\varepsilon}, a\right)$ in $L^{1}(\mathscr{O})$ uniformly in $\varepsilon$. On the other side $\varphi(y) F\left(\lambda, a_{k}(y)\right) \rightarrow \varphi(y) F(\lambda, a(y))$ in $L^{1}\left(\mathscr{O} \times \mathbb{R}^{N} ; \mu\right)$. Thus the convergence (3.1.4) in (a) extends to the present case.

(c) Consider next $\varphi$ and $a$ as in (b) and $F \in C_{o}^{0}$. Approximate $F$ in $L^{\infty}$ by $F_{k} \in C_{o}^{1}$. Then $\varphi F_{k}\left(u^{\varepsilon}, a\right) \rightarrow \varphi F\left(u^{\varepsilon}, a\right)$ in $L^{1}(\mathscr{O})$ uniformly in $\varepsilon$, and $\varphi(y) F_{k}(\lambda, a(y)) \rightarrow \varphi(y) F(\lambda, a(y))$ in $L^{1}\left(\mathscr{O} \times \mathbb{R}^{N} ; \mu\right)$. Thus formula (3.1.4) also extends to this case.

(d) Finally, for $\varphi, a, F$ as in the first assertion of the proposition, let $F_{k}(\lambda, \tau):=\zeta(\lambda / k, \tau / k) F(\lambda, \tau) \in C_{o}^{0}$ where $\zeta$ is a smooth cut off function, equal to one on the unit ball. Since $|F(\lambda, \tau)|=o\left(|\lambda|^{p}+|\tau|^{p}\right)$,

$$
\left|F(\lambda, \tau)-F_{k}(\lambda, \tau)\right| \leq \delta_{k}\left(|\lambda|^{p}+|\tau|^{p}\right)
$$

where $\delta_{k} \rightarrow 0$ as $k \rightarrow+\infty$. Using the $L^{p}$ estimates for $u^{\varepsilon}$ and $a$, it follows that $\varphi F_{k}\left(u^{\varepsilon}, a\right) \rightarrow \varphi F\left(u^{\varepsilon}, a\right)$ in $L^{1}$, uniformly in $\varepsilon$. Similarly, because $\varphi(y)\left\{|\lambda|^{p}+|a(y)|^{p}\right\} \in L^{1}(\mu), \varphi(y) F_{k}(\lambda, a(y)) \rightarrow \varphi(y) F(\lambda, a(y))$ in $L^{1}\left(\mathscr{O} \times \mathbb{R}^{N} ; \mu\right)$, and the first assertion of Proposition 3.1.4 follows.

(e) If $|F(\lambda, \tau)|=O\left(|\lambda|^{q}+|\tau|^{q}\right)$ at infinity, then $z^{\varepsilon}:=F\left(u^{\varepsilon}, a\right)$ is bounded in $L_{\text {comp }}^{p / q}(\overline{\mathscr{O}})$ and (3.1.4) implies that $z^{\varepsilon}$ converges weakly to

$$
\underline{z}(y):=\int F(\lambda, a(y)) \mu_{y}(d \lambda) \in L_{\text {comp }}^{p / q}(\overline{\mathscr{O}}) .
$$

Because $p / q>1$, the convergence

$$
\int z^{\varepsilon}(y) \varphi(y) d y \rightarrow \int \underline{z}(y) \varphi(y) d y
$$

extends to $\varphi \in L_{\text {comp }}^{r}(\overline{\mathscr{O}})$.

Corollary 3.1.5. Suppose that $u^{\varepsilon}$ is a pure bounded family in $L^{p}(\mathscr{O})$ with Young measure $\mu$, and $a \in L^{p}(\mathscr{O})$. Then $v^{\varepsilon}:=u^{\varepsilon}-a$ is a pure family in $L^{p}(\mathscr{O})$, and the associated measure $\nu$ is given by

$$
\iint \varphi(y) f(\lambda) \nu(y, d \lambda) d y=\iint \varphi(y) f(\lambda-a(y)) \mu(y, d \lambda) d y
$$

for all $\varphi \in C_{o}^{\infty}(\mathscr{O})$ and all $f \in C_{o}^{0}\left(\mathbb{R}^{N}\right)$.

Remark 3.1.6. Let $u^{\varepsilon}$ be a pure, real-valued and bounded family in $L^{p}(\mathscr{O})$, $p>1$. Then the mean value of $\mu$,

$$
m(y):=\int \lambda \mu(y, d \lambda) \text { a.e. }
$$


is the weak limit in $L^{p}$ of $u^{\varepsilon}$. The variance

$$
\begin{aligned}
\sigma(y) & :=\int|\lambda-m(y)|^{2} \mu(y, d \lambda) \\
& =\int \lambda^{2} \mu(y, d \lambda)-m(y)^{2} \text { a.e. }
\end{aligned}
$$

is defined and belongs to $L^{p / 2}(\mathscr{O})$, provided that $p \geq 2$. Then, saying that $\sigma \equiv 0$ on an open set $\mathscr{O}_{1} \subset \mathscr{O}$ means that, for almost all $y \in \mathscr{O}_{1}, \mu(y, d \lambda)$ is the probability measure concentrated at $\lambda=m(y)$, i.e. that $\mu(y, d \lambda)$ is the Dirac mass at $\lambda=m(y)$.

Proposition 3.1.7. Suppose that $u^{\varepsilon}$ is a pure bounded family in $L^{p}(\mathscr{O}), p \geq 2$, with Young measure $\mu$. Then the variance $\sigma$ vanishes on the bounded subset $\mathscr{O}_{1} \subset \mathscr{O}$, if and only if $u^{\varepsilon} \rightarrow m$ strongly in $L^{q}\left(\mathscr{O}_{1}\right)$ for all $q<p$.

Proof. Proposition 3.1.4 implies that for all $q<p$ and all $\varphi \in L_{\text {comp }}^{r}(\overline{\mathscr{O}})$

$$
\int \varphi(y)\left|u^{\varepsilon}(y)-m(y)\right|^{q} d y \rightarrow \iint \phi(y)|\lambda-m(y)|^{q} \mu(y, d \lambda) d y .
$$

If $\sigma=0$ on $\mathscr{O}_{1}$, then, for almost all $y \in \mathscr{O}_{1}, \mu(y, d \lambda)$ is the Dirac mass at $\lambda=m(y)$ and for $\varphi$ equal to the characteristic function of $\mathscr{O}_{1}$, the latter integral vanishes. In this case, (3.1.8) implies that $u^{\varepsilon} \rightarrow m$ strongly in $L^{q}\left(\mathscr{O}_{1}\right)$.

Conversely, if $u^{\varepsilon} \rightarrow m$ strongly in $L^{q}\left(\mathscr{O}_{1}\right)$ for some $q<p$, then for $\varphi \in$ $C_{o}^{0}\left(\mathscr{O}_{1}\right)$ the former integral in (3.1.8) tends to 0 . Therefore the latter vanishes, implying that $\mu(y, d \lambda)$ is the Dirac mass at $\lambda=m(y)$, at almost all points $y$ where $\varphi(y) \neq 0$.

Example 3.1.8. Let $u^{\varepsilon}$ be an oscillating family on $\mathscr{O} \subset \mathbb{R}^{n}$ of the form

$$
u^{\varepsilon}(y)=\mathscr{U}(y, \chi(y) / \varepsilon)
$$

where $\chi$ is a smooth $\mathbb{R}^{m}$-valued function with $d \chi \neq 0$ a.e. on $\mathscr{O}$, and $\mathscr{U}(y, \theta)$ a smooth and bounded function periodic in $\theta \in \mathbb{T}^{m}$. Then, this family is pure, and the associated Young measure is the image of $d y d \theta$ by $(y, \theta) \rightarrow(y, \lambda=$ $\mathscr{U}(y, \theta))$, i.e.

$$
\iint \varphi(y) f(\lambda) \mu(y, d \lambda) d y=\iint \varphi(y) f(\mathscr{U}(y, \theta)) d y d \theta
$$

It is understood that $d \theta$ is normalized to be of total mass 1 . We refer the reader to section 4 for a more systematic study of this situation. An important remark in this example is that the Young measure is independent of the phase function $\chi$.

Proposition 3.1.9. Let $u^{\varepsilon}$ be a pure bounded family in $L^{p}(\mathscr{O})$ with Young measure $\mu$. Let $v^{\varepsilon}$ be a bounded family in $L^{p}(\mathscr{O})$ which converges to zero strongly in $L_{\text {loc }}^{1}(\mathscr{O})$. Then $u^{\varepsilon}+v^{\varepsilon}$ is pure with Young measure $\mu$.

Proof. For any $f \in C_{o}^{1}$ and any $\varphi \in C_{o}^{\infty}, \varphi\left\{f\left(u^{\varepsilon}+v^{\varepsilon}\right)-f\left(u^{\varepsilon}\right)\right\}$ tends to 0 in $L^{1}(\mathscr{O})$.

3.2. Nonresonance and independence. We now turn to the situation described in section 2 . Recall that $\Omega$ denotes the half-space $] 0,+\infty\left[\times \mathbb{R}^{d}\right.$, and that $\Omega^{*}:=\Omega \cap\{x \neq 0\}$. For $T>0$, let $\left.\Omega_{T}:=\right] 0, T\left[\times \mathbb{R}^{d}\right.$. We are given a family $u^{\varepsilon}$ of solutions to (2.1.1), (2.1.2) which satisfies the estimates (2.1.4), (2.1.5). 
We assume that a subsequence has been extracted so that

$$
\left\{\begin{array}{l}
\text { the convergences }(2.2 .1)-(2.2 .5) \text { hold } \\
\text { the bounded families in } L^{2}\left(\Omega_{T}\right), v_{ \pm}^{\varepsilon}, \text { defined in }(2.2 .10) \text { are pure } \\
\text { the bounded family in } L^{p}(\Omega), \partial_{t} u^{\varepsilon}=v_{+}^{\varepsilon}+v_{-}^{\varepsilon}, \text { is pure } \\
\text { the families of Cauchy data } v_{0, \pm}^{\varepsilon}(2.2 .13) \text { are pure. }
\end{array}\right.
$$

Introduce

$$
w_{ \pm}^{\varepsilon}:=v_{ \pm}^{\varepsilon}-\underline{v}_{ \pm}=\frac{1}{2}\left(\partial_{t} \mp \partial_{r}\right)\left(u^{\varepsilon}-\underline{u}\right)
$$

where $\underline{v}_{ \pm}$is the weak limit in $L^{2}\left(\Omega_{T}\right)$ of $v_{ \pm}^{\varepsilon}$ which is, by (2.2.1),

$$
\underline{v}_{ \pm}=\frac{1}{2}\left(\partial_{t} \mp \partial_{r}\right) \underline{u} \text {. }
$$

Because $v_{ \pm}^{\varepsilon}$ satisfy (2.2.12) on $\Omega^{*}:=\Omega \backslash\{x=0\}, w_{ \pm}^{\varepsilon}$ satisfy on $\Omega^{*}$ the equations

$$
\begin{aligned}
2\left(\partial_{t} \pm \partial_{r}\right) w_{ \pm}^{\varepsilon} & +(d-1) r^{-1}\left(w_{+}^{\varepsilon}-w_{-}^{\varepsilon}\right) \\
& +F\left(\partial_{t} \underline{u}+w_{+}^{\varepsilon}+w_{-}^{\varepsilon}\right)-\underline{F}=r^{-2} \Delta_{\hat{x}}\left(u^{\varepsilon}-\underline{u}\right) .
\end{aligned}
$$

The estimates (2.1.4) and (2.1.5) imply that $w_{ \pm}^{\varepsilon}$ is bounded in $L^{2}\left(\Omega_{T}\right)$, $r^{-1} \Delta_{\hat{x}}\left(\mathcal{u}^{\varepsilon}-\underline{u}\right)$ is bounded in $L^{2}\left(\Omega_{T}\right)$ and $w_{+}^{\varepsilon}+w_{-}^{\varepsilon}$ is bounded in $L^{p}(\Omega)$. Thus $F\left(\partial_{t} \underline{u}+w_{+}^{\varepsilon}+w_{-}^{\varepsilon}\right)$ is bounded in $L^{p^{\prime}}(\Omega), p^{\prime}:=p /(p-1)$. Then, (3.2.3) yields

$$
\left(\partial_{t} \pm \partial_{r}\right) w_{ \pm}^{\varepsilon}=r^{-1} g_{ \pm}^{\varepsilon}+h_{ \pm}^{\varepsilon}
$$

with $g_{ \pm}^{\varepsilon}$ [resp. $h_{ \pm}^{\varepsilon}$ ] bounded in $L^{2}\left(\Omega_{T}\right)$ [resp. $L^{p^{\prime}}(\Omega)$ ]. Moreover, the uniform estimates (2.1.5) imply that

$$
\Gamma_{k, l} w_{ \pm}^{\varepsilon} \text { is bounded in } L^{2}\left(\Omega_{T}\right) .
$$

The Cauchy data for $w_{ \pm}^{\varepsilon}$ are

$$
w_{ \pm \mid t=0}^{\varepsilon}=w_{0, \pm}^{\varepsilon}:=v_{0, \pm}^{\varepsilon}-\underline{v}_{0, \pm}
$$

where $\underline{v}_{0, \pm}=\underline{u}_{1} \mp \partial_{r} \underline{u}_{0}$ is the weak limit of $v_{0, \pm}^{\varepsilon}$.

Corollary 3.1.5 and the assumptions on $v_{ \pm}^{\varepsilon}$ imply that the families $w_{ \pm}^{\varepsilon}$ are pure. Denote by $\mu_{ \pm}$their Young measures. They are measurable families of probability measures $\mu_{ \pm}(y, \cdot)$ on $\mathbb{R}$, parametrized by $y:=(t, x) \in \Omega$. Similarly the families $w_{0, \pm}^{\varepsilon}$ are pure and we denote by $\mu_{0, \pm}$ the Young measures which are measurable families of probabilities $\mu_{ \pm}(x, \cdot)$ on $\mathbb{R}$, parametrized by $x \in \mathbb{R}^{d}$. From the general results of section 3.1, we deduce the following facts.

Proposition 3.2.1. (i) $\lambda \in L^{2}\left(\Omega_{T} \times \mathbb{R}, \mu_{ \pm}\right)$for all $T>0$, and, $\lambda \in L^{2}\left(\mathbb{R}^{d} \times\right.$ $\mathbb{R}, \mu_{0, \pm}$.

(ii) The expectations of $\mu_{ \pm}$and $\mu_{0, \pm}$ vanish:

$$
\begin{gathered}
\int \lambda \mu_{ \pm}(y, d \lambda)=0, \quad \text { for almost all } y \in \Omega . \\
\int \lambda \mu_{0, \pm}(x, d \lambda)=0, \quad \text { for almost all } x \in \mathbb{R}^{d} .
\end{gathered}
$$


Proof. Part (i) is a consequence of the uniform $L^{2}$ estimates and of Proposition 3.1.3. Part (ii) follows from the weak convergence of $w_{ \pm}^{\varepsilon}$ to 0 and Remark 3.1.6.

The basic ingredient in our study is the following weak form of compensated compactness.

Theorem 3.2.2. Let $w^{\varepsilon}:=\left(w_{+}^{\varepsilon}, w_{-}^{\varepsilon}\right)$. Then the family $w^{\varepsilon}$ is pure, and the associated Young measure $\mu$ on $\Omega \times \mathbb{R}^{2}$ satisfies

$$
\mu\left(y, d \lambda_{1}, d \lambda_{2}\right)=\mu_{+}\left(y, d \lambda_{1}\right) \otimes \mu_{-}\left(y, d \lambda_{2}\right) \text { a.e. on } \Omega .
$$

Proof. (a) Let $W$ denote the space of $u \in L^{2}(\Omega)$ such that $\partial_{t} u$ and $\partial_{r} u$ belong to $L^{q}(\Omega)$ where $q>1$, and $\Gamma_{k, l} u \in L^{2}(\Omega)$ for all $(k, l)$. Then, for all $\omega \subset \subset \Omega^{*}$ the mapping $u \rightarrow u_{\mid \omega}$ is compact from $W$ to $L^{2}(\omega)$.

To prove this, one can assume that $\omega=] 0, T[\times\{a<|x|<b\}$ where $0<$ $a<b$. We use polar coordinates $(t, r=|x|, \hat{x})$. Expanding $u \in W$ into spherical harmonics, or smoothing $u$ in $\hat{x}$, we see that for all $\delta>0$ there is a $u_{1}$, smooth with respect to $\hat{x}$, such that

$$
\begin{gathered}
\left\|u-u_{1}\right\|_{L^{2}(\omega)} \leq \delta\|u\|_{W}, \quad\left\|\nabla_{\hat{x}} u_{1}\right\|_{L^{2}(\omega)} \leq C\|u\|_{W}, \\
\forall \hat{x} \in S^{d-1}:\left\|\nabla_{t, r} u_{1}(\cdot, \cdot, \hat{x})\right\|_{\left.L^{q}(] 0, T[\times] a, b\right]} \leq C(\delta)\|u\|_{W} .
\end{gathered}
$$

Choose an open ball $B \subset \mathbb{R}^{2}$ with $R \subset \subset B$. Choose a bounded linear extension operator $E: W^{1, q}(R) \rightarrow W_{o}^{1, q}(B)$. Let $j_{\varepsilon}$ denote a standard mollifier. Then $W_{o}^{1, q}(B)$ is compactly embedded into $L^{2}\left(\mathbb{R}^{2}\right)$ and $j_{\varepsilon} *$ converges strongly to the identity on $L^{2}\left(\mathbb{R}^{2}\right)$. Thus for any $\rho>0$ there are $\varepsilon>0$ and $C_{1}(\rho)$ such that $J u:=j_{\varepsilon} * E u$ satisfies

$$
\|J u-u\|_{L^{2}(R)} \leq \rho\|u\|_{W^{1, q}(R)}, \quad\|J u\|_{H^{1}(R)} \leq C_{1}(\rho)\|u\|_{W^{1, q}(R)} .
$$

Applying $J$ to $u_{1}(t, r, \hat{x})$ we find a smooth $u_{2}$ such that

$$
\left\|u_{2}-u_{1}\right\|_{L^{2}(\omega)} \leq \rho C(\delta)\|u\|_{W}, \quad\left\|\nabla_{t, r, \hat{x}} u_{1}\right\|_{L^{2}(\omega)} \leq C_{1}(\rho) C(\delta)\|u\|_{W} .
$$

Thus, for all $\varepsilon>0$ and all $u \in W$, one can find a $u_{2} \in H^{1}(\omega)$ such that

$$
\left\|u-u_{2}\right\|_{L^{2}(\omega)} \leq \varepsilon\|u\|_{W}, \quad\left\|u_{2}\right\|_{H^{1}(\omega)} \leq C(\varepsilon)\|u\|_{W} .
$$

Since the embedding $H^{1}(\omega) \subset L^{2}(\omega)$ is compact, the assertion follows.

(b) Suppose $z_{ \pm}^{\varepsilon}$ are bounded sequences in $L_{\text {loc }}^{2}(\Omega)$, which converge weakly to $\underline{z}_{ \pm}$and are such that

$$
\left\{\begin{array}{l}
\left(\partial_{t} \pm \partial_{r}\right) z_{ \pm}^{\varepsilon} \text { is bounded in } L_{\mathrm{loc}}^{q}\left(\Omega^{*}\right), \\
\Gamma_{k, l} z_{ \pm}^{\varepsilon} \text { is bounded in } L_{\mathrm{loc}}^{2}\left(\Omega^{*}\right),
\end{array}\right.
$$

where $q>1$. It follows that the product $z_{+}^{\varepsilon} z_{-}^{\varepsilon}$ converges weakly to $\underline{z}_{+} \underline{z}_{-}$on $\Omega^{*}$.

To prove this, one can act locally and assume that the functions $z_{ \pm}^{\varepsilon}$ are supported in a fixed compact subset of $\Omega^{*}$. The characteristic variety of the two systems $\mathscr{S}_{+}:=\left\{\partial_{t}+\partial_{r}, \Gamma_{k, l}\right\}$ and $\mathscr{S}_{-}:=\left\{\partial_{t}-\partial_{r}, \Gamma_{k, l}\right\}$ do not intersect. Introduce an even microlocal partition of unity,

$$
1=\sum_{k=1}^{3} \chi_{k}(y, \eta)
$$


with $\chi_{1}, \chi_{2}$ [resp. $\chi_{2}, \chi_{3}$ ] supported away from the characteristic variety of $\mathscr{S}_{+}\left[\right.$resp. $\left.\mathscr{S}_{-}\right]$. Thus $\chi_{1}\left(y, D_{y}\right) z_{+}^{\varepsilon}$ and $\chi_{2}\left(y, D_{y}\right) z_{+}^{\varepsilon}$ [resp. $\chi_{2}\left(y, D_{y}\right) z_{-}^{\varepsilon}$ and $\left.\chi_{3}\left(y, D_{y}\right) z_{+}^{\varepsilon}\right]$ are bounded in the space $W$ of part (a) thus converge strongly in $L^{2}$. Consequently, $\chi_{j}\left(y, D_{y}\right) z_{+}^{\varepsilon} \cdot \chi_{k}\left(y, D_{y}\right) z_{-}^{\varepsilon}$ converge weakly when $j \neq 3$ or $k \neq 1$. In addition, one can choose the partition so that for $(y, \eta)$ in the support of $\chi_{3}$ and $\left(y, \eta^{\prime}\right)$ in the support of $\chi_{1}$, one always has $\eta+\eta^{\prime} \neq 0$, except when $\eta=\eta^{\prime}=0$. This implies the weak convergence of $\chi_{3}\left(y, D_{y}\right) z_{+}^{\varepsilon}$. $\chi_{1}\left(y, D_{y}\right) z_{-}^{\varepsilon}$. Adding the different pieces, the claim follows.

(c) Suppose that $X$ is a smooth vector field and that $u \in L_{\text {loc }}^{1}$ is such that $X u \in L_{\text {loc }}^{1}$. Then for any $f \in C_{o}^{\infty}(\mathbb{R}), f(u) \in L^{\infty}, X f(u) \in L_{\text {loc }}^{1}$ and the chain rule $X f(u)=f^{\prime}(u) X u$ holds. The right-hand side makes sense as a product of $X u \in L_{\text {loc }}^{1}$ and $f^{\prime}(u) \in L^{\infty}$. This is again a local statement, which is proved by approximating $u$ by smooth functions $u_{\nu}$ such that $u_{\nu} \rightarrow u$ and $X u_{\nu} \rightarrow X u$ in $L_{\text {loc }}^{1}$.

(d) Suppose that $f_{ \pm} \in C_{o}^{\infty}(\mathbb{R})$. Because $w_{ \pm}^{\varepsilon}$ are pure families, $z_{ \pm}^{\varepsilon}:=f_{ \pm}\left(w_{ \pm}^{\varepsilon}\right)$ converges weakly to

$$
\underline{z}_{ \pm}(y)=\int f_{ \pm}(\lambda) \mu_{ \pm}(y, d \lambda)
$$

By (c) and (3.2.4), (3.2.5), the $z_{ \pm}^{\varepsilon}$ satisfy assumption (3.2.10) of (b). Therefore $z_{+}^{\varepsilon} z_{-}^{\varepsilon}$ converges weakly to $\underline{z}_{+} \underline{z}_{-}$on $\Omega^{*}$. This means that for any $\varphi \in C_{o}^{0}\left(\Omega^{*}\right)$

$$
\int \varphi(y) f_{+}\left(w_{+}^{\varepsilon}(y)\right) f_{-}\left(w_{-}^{\varepsilon}(y)\right) d y \rightarrow \int \varphi(y) \underline{z}_{+}(y) \underline{z}_{-}(y) d y .
$$

Fubini's theorem and (3.2.11) show that the latter integral is equal to

$$
\iiint \varphi(y) f_{+}\left(\lambda_{+}\right) f_{-}\left(\lambda_{-}\right) \mu_{+}\left(y, d \lambda_{+}\right) \mu_{-}\left(y, d \lambda_{-}\right) d y .
$$

Thus, we have proved that

$$
\int \varphi(y) f\left(w_{+}^{\varepsilon}(y), w_{-}^{\varepsilon}(y)\right) d y \rightarrow \iiint \varphi(y) f\left(\lambda_{1}, \lambda_{2}\right) \mu_{+}\left(y, d \lambda_{1}\right) \mu_{-}\left(y, d \lambda_{2}\right) d y
$$

for all $f \in C_{o}^{\infty}\left(\mathbb{R}^{2}\right)$ which are linear combinations of functions of the form $f\left(\lambda_{1}, \lambda_{2}\right)=f_{1}\left(\lambda_{1}\right) f_{2}\left(\lambda_{2}\right)$ and all $\varphi \in C_{o}^{\infty}\left(\Omega^{*}\right)$. By density, (3.2.10) extends to all $f \in C_{o}^{0}\left(\mathbb{R}^{2}\right)$. Moreover, for such $f$, the functions $f\left(w^{\varepsilon}(y)\right)$ are uniformly bounded, and formula (3.2.12) also extends to $\varphi \in C_{o}^{\infty}(\Omega)$.

This proves that the family $w^{\varepsilon}=\left(w_{+}^{\varepsilon}, w_{-}^{\varepsilon}\right)$ is pure with Young measure given by the right-hand side of (3.2.12). This is equivalent to (3.2.9).

Remark 3.2.3. By density, formula (3.2.12) extends to the continuous $f$ on $\mathbb{R}^{2}$ such that $f(\lambda)=o\left(|\lambda|^{2}\right)$.

We now give integrability properties with respect to the measure $\mu$. The third statement in the next proposition is rather surprising, because we have no information on the integrability of $\left|w_{ \pm}^{\varepsilon}\right|^{p}$. Only the sum $w_{+}^{\varepsilon}+w_{-}^{\varepsilon}$ is known to be bounded in $L^{p}$, but it turns out that this implies strong integrability properties for the individual measures $\mu_{-}$and $\mu_{+}$.

Proposition 3.2.4. One has the following integrability properties.

(i) $\lambda_{1}^{2}+\lambda_{2}^{2} \in L^{1}\left(\Omega_{T} \times \mathbb{R}^{2}, \mu\right)$ for all $T>0$.

(ii) $\lambda_{1}+\lambda_{2} \in L^{p}\left(\Omega \times \mathbb{R}^{2}, \mu\right)$. 
(iii) $\lambda \in L^{p}\left(\Omega \times \mathbb{R}, \mu_{+}\right)$and $\lambda \in L^{p}\left(\Omega \times \mathbb{R}, \mu_{-}\right)$.

Proof. (a) Point (i) follows from the boundedness of $w^{\varepsilon}$ in $L^{2}\left(\Omega_{T}\right)$ and Proposition 3.1.3.

(b) Let $f(\lambda)$ be continuous and such that $f(\lambda)=o\left(\lambda^{2}\right)$ at infinity and $s^{\varepsilon}:=$ $w_{+}^{\varepsilon}+w_{-}^{\varepsilon}$. Then, by Remark 3.2.3, one has

$$
\begin{gathered}
\int \varphi(y) f\left(s^{\varepsilon}(y)\right) d y \rightarrow \iiint \varphi(y) f\left(\lambda_{1}+\lambda_{2}\right) \mu_{+}\left(y, d \lambda_{1}\right) \mu_{-}\left(y, d \lambda_{2}\right) d y \\
=\iiint \varphi(y) f\left(\lambda_{1}+\lambda_{2}\right) \mu\left(y, d \lambda_{1}, d \lambda_{2}\right) d y
\end{gathered}
$$

This proves that the family $s^{\varepsilon}$ is pure and that the associated measure $\nu$ is given by

$$
\int f(\tau) \nu(y, d \tau)=\iint f\left(\lambda_{1}+\lambda_{2}\right) \mu\left(y, d \lambda_{1}, d \lambda_{2}\right) \quad \text { a.e. on } \Omega .
$$

On the other hand, because $s^{\varepsilon}$ is bounded in $L^{p}(\Omega)$, we know from Proposition 3.1.3 that $\tau \in L^{p}(\Omega \times \mathbb{R}, \nu)$. Thus Fatou's lemma implies that $\lambda_{1}+\lambda_{2} \in$ $L^{p}\left(\Omega \times \mathbb{R}^{2}, \mu\right)$.

(c) For $\rho>0$, let

$$
f_{\rho}\left(\lambda_{1}, \lambda_{2}\right):=\left\{1+\rho\left|\lambda_{2}\right|^{p}\right\}^{-1}\left|\lambda_{1}+\lambda_{2}\right|^{p} .
$$

Because $p \geq 2$, the map $\tau \rightarrow|\tau|^{p}$ is $C^{2}$ and convex, so

$$
\left|\lambda_{1}+\lambda_{2}\right|^{p} \geq\left|\lambda_{2}\right|^{p}+p \lambda_{1} \lambda_{2}\left|\lambda_{2}\right|^{p-2} \text {. }
$$

Thus

$$
f_{\rho}\left(\lambda_{1}, \lambda_{2}\right) \geq\left\{1+\rho\left|\lambda_{2}\right|^{p}\right\}^{-1}\left|\lambda_{2}\right|^{p}+g_{\rho}\left(\lambda_{1}, \lambda_{2}\right)
$$

where

$$
g_{\rho}\left(\lambda_{1}, \lambda_{2}\right):=p \lambda_{1} \lambda_{2}\left\{1+\rho\left|\lambda_{2}\right|^{p}\right\}^{-1}\left|\lambda_{2}\right|^{p-2} .
$$

Because of (ii), $f_{\rho}$ is integrable with respect to $\mu$, and

$$
\iint f_{\rho}(\lambda) \mu(y, d \lambda) d y \leq C:=\iint\left|\lambda_{1}+\lambda_{2}\right|^{p} \mu(y, d \lambda) d y<+\infty .
$$

On the other hand, for each $\rho, g_{\rho}(\lambda)=\lambda_{1} \times O\left(\left|\lambda_{2}\right|\right)$ so that $g_{\rho} \in L^{1}\left(\Omega_{T} \times \mathbb{R}^{2}, \mu\right)$ by part (i). Now, Fubini's theorem and (3.2.7) imply that

$$
\iiint_{\Omega_{T} \times \mathbb{R}^{2}} g_{\rho}\left(\lambda_{1}, \lambda_{2}\right) \mu(y, d \lambda) d y=0 .
$$

Thus, integrating (3.2.15) over $\Omega_{T} \times \mathbb{R}^{2}$ and using Fubini's theorem yield

$$
\iint_{\Omega_{T} \times \mathbb{R}}\left\{1+\rho\left|\lambda_{2}\right|^{p}\right\}^{-1}\left|\lambda_{2}\right|^{p} \mu_{-}\left(y, d \lambda_{2}\right) d y \leq C .
$$

We can now let $\rho$ tend to 0 and $T$ tend to $+\infty$. Fatou's lemma implies that

$$
\iiint\left|\lambda_{2}\right|^{p} \mu_{-}(y, d \lambda) d y \leq C,
$$

and the proof of Proposition 3.2.4 is complete.

Another consequence of Theorem 3.2.2 is that one can express the weak limit $\underline{F}$ of $F\left(\partial_{t} u^{\varepsilon}\right)$ in terms of the Young measures $\mu_{ \pm}$. For later use, we state a slightly more general result. Introduce the notation

$$
\widetilde{F}(y, \lambda):=F\left(\partial_{t} \underline{u}(y)+\lambda\right)
$$

where $F$ is the nonlinearity of (2.1.1). Then $F\left(\partial_{t} u^{\varepsilon}\right)=\widetilde{F}\left(y, w_{+}^{\varepsilon}+w_{-}^{\varepsilon}\right)$. 
Proposition 3.2.5. $\widetilde{F}\left(y, \lambda_{1}+\lambda_{2}\right) \in L^{p^{\prime}}\left(\Omega \times \mathbb{R}^{2}, \mu\right)$ where $p^{\prime}:=p /(p-1)$ is the conjugate exponent of $p$. Moreover, for $\varphi \in C_{o}^{\infty}(\bar{\Omega}), f \in C_{o}^{\infty}(\mathbb{R})$ and $\varepsilon \rightarrow 0$, one has

(3.2.19)

$\int \varphi f\left(w_{+}^{\varepsilon}\right) \widetilde{F}\left(y, w_{+}^{\varepsilon}+w_{-}^{\varepsilon}\right) d y \rightarrow \iiint \varphi(y) f\left(\lambda_{1}\right) \widetilde{F}\left(y, \lambda_{1}+\lambda_{2}\right) \mu\left(y, d \lambda_{1}, d \lambda_{2}\right) d y$.

Proof. For bounded $\widetilde{F},(3.2 .19)$ is a consequence of Proposition 3.1.4. On the other hand, we know that $|F(\tau)|=O\left(|\tau|^{p-1}\right)$. The result follows as in Proposition 3.1 .4 by approximating $\widetilde{F}$ by $\widetilde{F}_{k}:=\zeta\left\{\left(\lambda_{1}+\lambda_{2}\right) / k\right\} \widetilde{F}$, using that $\partial_{t} \underline{u} \in L^{p}(\Omega), w_{+}^{\varepsilon}+w_{-}^{\varepsilon}$ are bounded in $L^{p}(\Omega)$, and $\lambda_{1}+\lambda_{2} \in L^{p}(\mu)$.

Remark 3.2.6. Taking $f \equiv 1$ in (3.2.19), we see that the weak limit of $F\left(\partial_{t} u^{\varepsilon}\right)$ is given by

$$
\underline{F}(y)=\int \widetilde{F}\left(y, \lambda_{1}+\lambda_{2}\right) \mu_{+}\left(y, d \lambda_{1}\right) \mu_{--}\left(y, d \lambda_{2}\right) \quad \text { a.e. on } \Omega .
$$

Using the ideas of Proposition 3.2.4 and the notations of Definition 2.3.1, one can also improve the results of Proposition 3.1.7. We use the following lemma.

Lemma 3.2.7. If $f_{n}$ and $g_{n}$ are bounded families in $L^{2}(\omega)$ such that

(i) $f_{n}+g_{n}$ is bounded in $L^{p}(\omega)$ for some $p>2$,

(ii) $f_{n}$ converges strongly to 0 in $L^{q}(\omega)$ for all $q<2$,

(iii) $f_{n} g_{n}$ converges weakly to zero in $\mathscr{D}^{\prime}(\omega)$, then $f_{n}$ converges strongly to zero in $L_{\text {loc }}^{2}(\omega)$.

Proof. Let $h_{n}:=f_{n}+g_{n}$, bounded in $L^{p}(\omega)$. For all $\varphi \in C_{o}^{\infty}(\omega)$,

$$
\int \varphi\left(f_{n}\right)^{2} d y=\int \varphi f_{n} h_{n} d y-\int \varphi f_{n} g_{n} d y
$$

The second term on the right converges to zero by hypothesis (iii). The first converges to 0 since $f_{n}$ converges to 0 in $L^{q}$ and $h_{n}$ is bounded in $L^{p}$ for $q=p /(p-1)<2$.

Proposition 3.2.8. Let $\sigma_{ \pm}(y)$ denote the variance of $\mu_{ \pm}(y, \cdot)$, i.e.

$$
\sigma_{ \pm}(y):=\int_{\mathbb{R}} \lambda^{2} \mu_{ \pm}(y, d \lambda) \text { a.e. on } \Omega \text {. }
$$

If $\sigma_{+}(y)=0$ [resp. $\sigma_{-}(y)=0$ ] on a neighborhood of $y \in \Omega$, then the family $w_{+}^{\varepsilon}\left[\right.$ resp. $\left.w^{\varepsilon}\right]$ is compact in $L^{q}$ at $\underline{y}$, for all $q<2$. In addition, if $\underline{y} \in \Omega^{*}$, then the family $w_{+}^{\varepsilon}$ [resp. $\left.w_{-}^{\varepsilon}\right]$ is compact in $L^{2}$ at $\underline{y}$.

Proof. (a) The first statement is a special case of Proposition 3.1.7.

(b) Apply Lemma 3.2.7 to $w_{+}^{\varepsilon}$ and $w_{-}^{\varepsilon}$. We know that $s^{\varepsilon}:=w_{+}^{\varepsilon}+w_{-}^{\varepsilon}$ is bounded in $L^{p}(\Omega)$. If $\sigma_{-}(y)$ vanishes on $\omega \subset \subset \Omega^{*}$, then $w_{+}^{\varepsilon}$ converges strongly to zero in $L^{q}(\omega)$ for all $q<2$. Theorem 3.2 .2 implies that $w_{+}^{\varepsilon} w_{-}^{\varepsilon}$ converges weakly to 0 in $\mathscr{D}^{\prime}(\Omega)$. Thus Lemma 3.2.7 implies that if $\sigma_{-}(y)$ vanishes on $\omega \subset \subset \Omega^{*}$, then $w_{+}^{\varepsilon}$ converges strongly to zero in $L_{\text {loc }}^{2}(\omega)$.

3.3. Transport equations for the Young measures. In the last section we only used the a priori estimates and the reduced form (3.2.4) of the equations. We now take advantage of the full expression (3.2.3) of the equations. 
Theorem 3.3.1. In $\Omega^{*} \times \mathbb{R}$, the Young measures $\mu_{ \pm}$satisfy

$$
\begin{gathered}
2\left(\partial_{t} \pm \partial_{r}\right) \mu_{ \pm} \mp(d-1) r^{-1} \partial_{\lambda}\left\{\lambda \mu_{\mu}\right\}-\partial_{\lambda}\left\{F_{ \pm}(y, \lambda) \mu_{ \pm}\right\}=0, \\
\mu_{ \pm \mid t=0}=\mu_{0, \pm},
\end{gathered}
$$

where

$$
F_{ \pm}(y, \lambda):=\int \widetilde{F}(y, \lambda+\tau) \mu_{\mp}(y, d \tau)-\underline{F}(y) .
$$

Recall that Corollary 3.2.7 expresses $\underline{F}$ in terms of $\widetilde{F}$ and $\mu_{ \pm}$. In particular,

$$
\int F_{+}(y, \lambda) \mu_{+}(y, d \lambda)=\int F_{-}(y, \lambda) \mu_{-}(y, d \lambda)=0, \quad \text { a.e. on } \Omega \text {. }
$$

The system (3.3.1) is nonlinear, because $F_{ \pm}$depends on $\mu_{+}$and $\mu_{-}$. Moreover, the two equations are coupled through the $F_{ \pm}$terms.

Remark 3.3.2. One has $\left|\widetilde{F}\left(y, \lambda_{1}+\lambda_{2}\right)\right| \leq C\left(\left|\partial_{t} \underline{u}(y)\right|^{p-1}+\left|\lambda_{1}\right|^{p-1}+\left|\lambda_{2}\right|^{p-1}\right)$. Therefore, Fubini's theorem and Hölder's inequality, together with the estimates of Proposition 3.2.5, imply that

$$
\left|\widetilde{F}_{ \pm}(y, \lambda)\right| \leq C\left(g_{ \pm}(y)+|\lambda|^{p-1}\right)
$$

where $g_{ \pm} \in L^{p^{\prime}}(\Omega)$. This implies that $F_{ \pm} \in L^{p^{\prime}}\left(\Omega \times \mathbb{R}, \mu_{ \pm}\right)$.

Thus, $F_{ \pm}$is $\mu_{ \pm}$integrable, at least locally in $y$, and the product $F_{ \pm} \mu_{ \pm}$is well defined as a distribution on $\Omega \times \mathbb{R}$ so that equation (3.3.1) makes sense.

Moreover, equation (3.3.1) implies that $\mu_{+}$and $\mu_{-}$have traces on $t=0$, at least in the sense of distribution on $\mathbb{R}^{d} \backslash\{0\}$. The initial conditions (3.3.2) are interpreted in this sense.

Proof of Theorem 3.3.1. We give the proof for $\mu_{+}$. For $f \in C_{o}^{\infty}(\mathbb{R})$ and $\varphi \in C_{o}^{\infty}\left(\mathbb{R} \times \mathbb{R}^{d} \backslash\{0\}\right)$, multiply equation (3.2.3) by $\varphi(y) f^{\prime}\left(w_{ \pm}^{\varepsilon}\right)$. The strategy is integrate by parts and pass to the limit $\varepsilon \rightarrow 0$ in each term. The resulting expression is the weak form of equations (3.3.1), (3.3.2).

(a) For the first term of (3.2.3), recall from (3.2.4) that

$$
2\left(\partial_{t}+\partial_{r}\right) w_{+}^{\varepsilon}=g^{\varepsilon} \in r^{-1} L^{1}\left(\Omega_{T}\right), \quad w_{+\mid t=0}^{\varepsilon}=w_{0,+}^{\varepsilon} .
$$

Approximating $w_{+}^{\varepsilon}$ by smooth functions and passing to the limit yield that $f\left(w_{+}^{\varepsilon}\right)$ satisfies

$$
2\left(\partial_{t}+\partial_{r}\right) f\left(w_{+}^{\varepsilon}\right)=g^{\varepsilon} f^{\prime}\left(w_{+}^{\varepsilon}\right), \quad f\left(w_{+}^{\varepsilon}\right)_{\mid t=0}=f\left(w_{0,+}^{\varepsilon}\right),
$$

and

$$
\int_{\Omega} g^{\varepsilon} f^{\prime}\left(w_{+}^{\varepsilon}\right) \varphi d y=-2 \int_{\Omega} f\left(w_{+}^{\varepsilon}\right)\left(\partial_{t}+\partial_{r}\right) \varphi d y-2 \int_{\mathbb{R}^{d}} f\left(w_{0,+}^{\varepsilon}\right) \varphi_{\mid t=0} d x .
$$

The definition of Young measure yields an expression for the limit of the righthand side, namely

$$
\begin{aligned}
& -2 \int_{\Omega \times \mathbb{R}}\left(\partial_{t}+\partial_{r}\right) \varphi(y) f(\lambda) \mu_{+}(y, d \lambda) d y \\
& \quad-2 \int_{\mathbb{R}^{d} \times \mathbb{R}} \varphi(0, x) f(\lambda) \mu_{0,+}(x, d \lambda) d x .
\end{aligned}
$$


(b) For the $r^{-1} w_{+}^{\varepsilon}$ term in (3.2.3), the definition of Young measure yields

$$
\lim \int_{\Omega} r^{-1} \varphi f^{\prime}\left(w_{+}^{\varepsilon}\right) w_{+}^{\varepsilon} d y=\int_{\Omega \times \mathbb{R}} r^{-1} \varphi(y) \lambda f^{\prime}(\lambda) \mu_{+}(y, d \lambda) d y .
$$

(c) For the $r^{-1} w_{+}^{\varepsilon}$ term, Theorem 3.2 .3 yields

$$
\begin{aligned}
& \lim \int_{\Omega} r^{-1} \varphi f^{\prime}\left(w_{+}^{\varepsilon}\right) w_{-}^{\varepsilon} d y \\
& \quad=\int_{\Omega \times \mathbb{R} \times \mathbb{R}} \varphi(y) \lambda_{2} f^{\prime}\left(\lambda_{1}\right) \mu_{+}\left(y, d \lambda_{1}\right) \mu_{-}\left(y, d \lambda_{2}\right) d y=0
\end{aligned}
$$

since (3.2.7) implies that the integral in $\lambda_{2}$ vanishes.

(d) For the $F$ term in (3.2.3), Propositions 3.2.5, 3.2.6 and Theorem 3.2.2 yield

$$
\begin{aligned}
& \lim \int_{\Omega} \varphi f^{\prime}\left(w_{+}^{\varepsilon}\right)\left\{F\left(\partial_{t} \underline{u}+w_{+}^{\varepsilon}+w_{-}^{\varepsilon}\right)-\underline{F}\right\} d y \\
& \quad=\int_{\Omega \times \mathbb{R} \times \mathbb{R}} \varphi(y) f^{\prime}(\lambda) F\left(\partial_{t} \underline{u}(y)+\lambda_{+}+\lambda_{-}\right) \mu_{+}\left(y, d \lambda_{+}\right) \mu_{-}\left(y, d \lambda_{-}\right) d y .
\end{aligned}
$$

The function $F_{+}$is defined so that the integral in $\lambda_{-}$yields

$$
\begin{array}{r}
\lim \int_{\Omega} \varphi f^{\prime}\left(w_{+}^{\varepsilon}\right)\left\{F\left(\partial_{t} \underline{u}+w_{+}^{\varepsilon}+w_{-}^{\varepsilon}\right)-\underline{F}\right\} d y \\
\quad=\int_{\Omega \times \mathbb{R}} \varphi(y) f^{\prime}(\lambda) F_{+}(y, \lambda) \mu_{+}(y, d \lambda) d y
\end{array}
$$

(e) For the $r^{-2} \Delta_{\hat{x}}\left(u^{\varepsilon}-\underline{u}\right)$ term in (3.2.3), write $\Delta_{\hat{x}}$ as $\sum \Gamma_{k, l}^{2}$ and integrate by parts in the integral. This is justified since $\Gamma_{k, l} w_{+}^{\varepsilon}$ belongs to $L^{2}\left(\Omega_{T}\right)$ and is bounded in this space. This procedure leads to a sum of terms of the form

$$
\int_{\Omega} g_{k, l}^{\varepsilon} \Gamma_{k, l}\left(u^{\varepsilon}-\underline{u}\right) d y
$$

with $g_{k, l}^{\varepsilon}:=-\Gamma_{k, l}\left\{r^{-2} \varphi f^{\prime}\left(w_{+}^{\varepsilon}\right)\right\}$. These $g_{k, l}^{\varepsilon}$ are bounded families in $L^{2}$. The strong convergence $(2.2 .2)$ asserts that $\Gamma_{k, l}\left(u^{\varepsilon}-\underline{u}\right) \rightarrow 0$ in $L_{\text {loc }}^{2}(\Omega)$. Therefore the integrals (3.3.10) tend to 0 as $\varepsilon$ tends to 0 and

$$
\lim \int_{\Omega} r^{-2} \varphi f^{\prime}\left(w_{+}^{\varepsilon}\right) \Delta_{\omega}\left(u^{\varepsilon}-\underline{u}\right) d y=0 .
$$

(f) Setting the sum of the expressions for the limits in equations (3.3.6)(3.3.9) and (3.3.11) equal to 0 yields

$$
\begin{aligned}
0= & -2 \int_{\Omega \times \mathbb{R}}\left\{\left(\partial_{t}+\partial_{r}\right) \varphi\right\} f(\lambda) \mu_{+}(y, d \lambda) d y \\
& -2 \int_{\mathbb{R}^{d} \times \mathbb{R}} \varphi(0, x) f(\lambda) \mu_{0,+}(x, d \lambda) d x \\
& +(d-1) \int_{\Omega \times \mathbb{R}} r^{-1} \varphi(y) \lambda f^{\prime}(\lambda) \mu_{+}(y, d \lambda) d y \\
& +\int_{\Omega \times \mathbb{R}} \varphi(y) f^{\prime}(\lambda) F_{+}(y, \lambda) \mu_{+}(y, d \lambda) d y .
\end{aligned}
$$

This is the weak form of (3.3.1), (3.3.2) and the proof is complete.

An interesting corollary of this result is a transport inequality for the variances $\sigma_{ \pm}$of $\mu_{ \pm}$. 
Theorem 3.3.2. Let $\sigma_{ \pm}$denote the variance of $\mu_{ \pm}$,

$$
\sigma_{ \pm}(y):=\int_{\mathbb{R}} \lambda^{2} \mu_{ \pm}(y, d \lambda) \text { a.e. on } \Omega \text {. }
$$

Then $\sigma_{ \pm} \in L^{1}\left(\Omega_{T}\right) \cap L^{p / 2}(\Omega),\left(\partial_{t} \pm \partial_{r}\right) \sigma_{ \pm} \in r^{-1} L^{p / 2}(\Omega)+L^{1}(\Omega)$ and there is a constant $c>0$ such that

$$
\left(\partial_{t} \pm \partial_{r}\right) \sigma_{ \pm} \pm(d-1) r^{-1} \sigma_{ \pm}+c\left(\sigma_{ \pm}\right)^{p / 2} \leq 0, \text { a.e. on } \Omega^{*} \text {. }
$$

Moreover, the trace of $\sigma_{ \pm}$on $t=0$ is the variance $\sigma_{0, \pm}$ of $u_{0, \pm}$ with respect to $\lambda$.

Proof. We give the proof for $\sigma_{+}$. That $\sigma_{+} \in L^{1}\left(\Omega_{T}\right) \cap L^{p / 2}(\Omega)$ is a consequence of Proposition 3.2.1 and Proposition 3.2.4.

The idea is to extend (3.3.12) to $f(\lambda)=\lambda^{2} / 2$. We emphasize here that one cannot make a direct proof, i.e. multiply equation (3.2.3) by $w_{+}^{\varepsilon}$, because the multiplication of $\left.F\left(\partial_{t} \underline{u}+w_{+}^{\varepsilon}+w_{-}^{\varepsilon}\right)\right) \in L^{p^{\prime}}$ by $w_{+}^{\varepsilon} \in L^{2}$ does not make sense.

Fix $\varphi \in C_{o}^{\infty}\left(\mathbb{R} \times \mathbb{R}^{d} \backslash\{0\}\right)$. Choose $\zeta \in C_{o}^{\infty}(\mathbb{R})$ such that $\zeta=1$ on $[-1,+1]$. For $k \geq 1$, let

$$
f_{k}(\lambda):=\frac{1}{2} \lambda^{2} \zeta(\lambda / k) \in C_{o}^{\infty}(\mathbb{R}) .
$$

One has the uniform estimates

$$
\left|f_{k}(\lambda)\right| \leq \lambda^{2} / 2, \quad\left|f_{k}^{\prime}(\lambda)\right| \leq C|\lambda| .
$$

Proposition 3.2.1 implies that

$$
\begin{gathered}
\left\{\left(\partial_{t}-\partial_{r}\right) \varphi\right\} \lambda^{2} \in L^{1}\left(\Omega \times \mathbb{R}, \mu_{+}\right), \quad r^{-1} \varphi \lambda^{2} \in L^{1}\left(\Omega \times \mathbb{R}, \mu_{+}\right), \\
\varphi(0, \cdot) \lambda^{2} \in L^{1}\left(\mathbb{R}^{d} \times \mathbb{R}, \mu_{0,+}\right) .
\end{gathered}
$$

Proposition 3.2.4 and Remark 3.3.2 imply that

$$
\varphi(y) \lambda F_{+}(y, \lambda) \in L^{1}\left(\Omega \times \mathbb{R}, \mu_{+}\right) .
$$

Apply (3.3.12) with $f_{k}$. Thanks to (3.3.16)-(3.3.18), we can use Lebesgue's dominated convergence theorem to pass to the limit in $k$. This proves that (3.3.12) holds for $f(\lambda)=\lambda^{2} / 2$. Integrating with respect to $\lambda$, this shows that, for all $\varphi \in C_{o}^{\infty}\left(\mathbb{R} \times \mathbb{R}^{d} \backslash\{0\}\right)$,

$$
\begin{gathered}
-\int_{\Omega}\left\{\left(\partial_{t}+\partial_{r}\right) \varphi\right\} \sigma_{+}(y) d y+(d-1) \int_{\Omega} r^{-1} \varphi(y) \sigma_{+}(y) d y \\
+\int_{\Omega} \varphi(y) h_{+}(y) d y-\int_{\mathbb{R}} \varphi(0, x) \sigma_{0,+}(x) d x=0
\end{gathered}
$$

where

$$
h_{+}(y):=\int_{\mathbb{R}} \lambda F_{+}(y, \lambda) \mu_{+}(y, d \lambda) .
$$

Now, formula (3.3.19) implies that

$$
\left(\partial_{t}+\partial_{r}\right) \sigma_{+}+(d-1) r^{-1} \sigma_{+}+h_{+}=0 \text { on } \Omega^{*} .
$$

Because of Proposition 3.2.4 and Remark 3.3.2, $\lambda F_{+}(y, \lambda)$ is $\mu_{+}$integrable and therefore $h_{+} \in L^{1}(\Omega)$. Hence (3.3.21) implies that $\left(\partial_{t}+\partial_{r}\right) \sigma_{+} \in r^{-1} L^{p / 2}(\Omega)+$ 
$L^{1}(\Omega)$. This implies that $\sigma_{+}$has a trace at $t=0$, on $\mathbb{R}^{d} \backslash\{0\}$. Then (3.3.19) shows that this trace must be $\sigma_{0,+}$.

It remains to show that

$$
h_{+}(y) \geq c\left(\sigma_{+}(y)\right)^{p / 2}, \quad \text { a.e. on } \Omega \text {. }
$$

Because the expectation of $\mu_{+}$is 0 , one has

$$
h_{+}(y):=\int_{\mathbb{R} \times \mathbb{R}} \lambda\{\widetilde{F}(y, \lambda+\tau)-\widetilde{F}(y, \tau)\} \mu_{+}(y, d \lambda) \mu_{-}(y, d \tau) .
$$

The fact that $h$ is nonnegative follows from the monotoniticity of $F$. We use a quantitative form. Notice that, for any $\lambda \neq 0$, the function $a \rightarrow$ $\lambda\{F(a+\lambda)-F(a)\}$ has positive (resp. negative) derivative on $]-\lambda / 2,+\infty[$ (resp. ] $-\infty,-\lambda / 2[$ ) so its minimum is attained at $a=-\lambda / 2$ which yields

$$
\forall \lambda \in \mathbb{R}, \forall a \in \mathbb{R}: \lambda\{F(a+\lambda)-F(a)\} \geq|\lambda|^{p} / 2^{p-2} .
$$

This inequality together with (3.3.23) implies that

$$
h_{+}(y) \geq \int_{\mathbb{R} \times \mathbb{R}} 2^{2-p}|\lambda|^{p} \mu_{+}(y, d \lambda) \mu_{-}(y, d \tau)
$$

and (3.2.22) follows, finishing the proof of Theorem 3.3.7.

3.4. Proof of the compactness and absorption theorems of section 2.3. Let $u^{\varepsilon}$ be a family of solutions of (2.1.1), satisfying estimates (2.1.4), (2.1.5). To prove compactness, one must show that every subsequence has a convergent subsequence. Passing to a subsequence of a given subsequence, we may assume that conditions (3.2.1) are fulfilled.

Suppose that the Cauchy data $v_{0, \pm}^{\varepsilon}$ are compact at $x_{0} \neq 0$. Since the $\hat{v}_{0, \pm}^{\varepsilon}$ converge weakly, it follows that they converge strongly on a neighborhood of $x_{0}$. Proposition 3.1.7 implies that the variance of the Young measure of $v_{0, \pm}^{\varepsilon}$ vanishes a.e. around $x_{0}$. By Corollary 3.1.5, this variance is equal to $\sigma_{0, \pm}$, the variance of the measure $\mu_{0, \pm}$ associated to $w_{ \pm}^{\varepsilon}$. Choose a neighborhood $\omega_{0}$ of $x_{0}$ such that $\sigma_{0, \pm}$ vanishes on $\omega_{0}$.

To prove Theorem 2.3.2(ii), Proposition 3.2.8 shows that it suffices to prove that the variance of the Young measures of $v_{-}^{\varepsilon}$ vanish on a neighborhood of the points $x=x_{o}-t \hat{x}_{o}$, for $0<t<\left|x_{o}\right|$. Note that these are the points of the part of the integral curve of $\left(\partial_{t}-\partial_{r}\right)$ which passes through $\left(0, x_{o}\right)$ and stops just before reaching the origin. Let

$$
\omega_{-}:=\left\{\left(t, x=x_{1}-t \hat{x}_{1}\right)|0<t<| x_{1} \mid, x_{1} \in \omega_{0}\right\} \subset \Omega^{*} .
$$

Thanks to Proposition 3.2.8, it suffices to show that $\sigma_{-}$vanishes on $\omega_{-}$. To do this, it suffices to remark that $\sigma_{-} \geq 0$ satisfies (3.3.14) in $\omega_{-}$and has vanishing initial data on $\omega_{0}$.

Similarly, if $\sigma_{0,+}$ vanishes on $\omega_{0}$, then (3.3.14) implies that $\sigma_{+}$vanishes on

$$
\omega_{+}:=\left\{\left(t, x=x_{1}+t \hat{x}_{1}\right) \mid 0<t, x_{1} \in \omega_{0}\right\} \subset \Omega^{*} .
$$

This implies Theorem 2.3.2(i), completing the proof.

In the same way, the compactness result stated in Theorem 2.3.3 is a consequence of the following result. 
Proposition 3.4.1. If $(p-2)(d-1) \geq 2$, then $\sigma_{+}=0$ a.e. in $\mathscr{C}:=\{(t, x) \mid t>$ $|x|\}$.

Proof. Introduce polar coordinates $x=r \hat{x}$ on $\mathbb{R}^{d}$, and change variables

$$
(t, r, \hat{x}) \rightarrow(s:=t-r, r, \hat{x}) \text {. }
$$

Then $\mathscr{C} \cap \Omega^{*}$ is transformed to $\left\{s>0, r>0, \hat{x} \in S^{d-1}\right\}$.

Theorem 3.3.7 implies that $\sigma_{+} \in L^{1}\left(\Omega_{T}\right) \cap L^{p / 2}(\Omega),\left(\partial_{t}+\partial_{r}\right) \sigma_{+} \in r^{-1} L^{p / 2}(\Omega)$ $+L^{1}(\Omega)$ and (3.3.14) holds on $\Omega^{*}$. Thus for almost all $s>0$ and $\hat{x} \in S^{d-1}$, the function

$$
y(r):=\sigma_{+}(s+r, r \hat{x})
$$

satisfies

$$
\begin{cases}y \in L_{\mathrm{loc}}^{1} \cap L_{\mathrm{loc}}^{p / 2}(] 0,+\infty[), & y^{\prime} \in L_{\mathrm{loc}}^{1}(] 0,+\infty[), \\ y^{\prime}+(d-1) r^{-1} y+c y^{p / 2} \leq 0 & \text { on }] 0,+\infty[, \\ y \geq 0 \text { on }] 0,+\infty[. & \end{cases}
$$

It suffices to show that, when $\beta:=(p-2)(d-1) \geq 2$, the only solution to (3.4.5) is $y \equiv 0$. Introduce

$$
z(r):=r^{(d-1)} y(r) .
$$

Then $z$ is locally absolutely continuous on $] 0,+\infty[$, nonnegative and satisfies

$$
z^{\prime}+c r^{-\beta} z^{p / 2} \leq 0 .
$$

The first consequence of (3.4.7) is that $z$ is nonincreasing. Assume that, for some $\rho>0, z(\rho)>0$. Then $z(r) \geq z(\rho)>0$ for $r \in] 0, \rho]$. Thus on $] 0, \rho]$

$$
\frac{d}{d r}\left\{z(r)^{1-p / 2}\right\}=(1-p / 2) z^{\prime} z^{-p / 2} \leq c\left(\frac{p}{2}-1\right) r^{-\beta}
$$

Since $\beta \geq 1, p>2$ the integral of the right-hand side over $[r, \rho]$ diverges to $-\infty$ as $r$ tends to zero. Thus $z(\rho)^{1-p / 2}-z(r)^{1-p / 2} \rightarrow-\infty$ as $r$ tends to zero, which contradicts $z(r) \geq z(\rho)$. Thus one must have $z(\rho)=0$ and therefore $y(\rho)=0$ for all $\rho>0$ and the proof is complete.

Theorem 2.3.4, is based upon the link between compactness for $\nabla u^{\varepsilon}$ and compactness for $v_{ \pm}^{\varepsilon}$.

Lemma 3.4.2. (i) If $\sigma_{+}$and $\sigma_{-}$vanish a.e. in a neighborhood of $y:=(t, x) \in$ $\Omega$, then $\nabla_{t, x} u^{\varepsilon}$ converges strongly in $L^{2}$ near $y$ if $x \neq 0$ and strongly in $L^{q}$ for all $q<2$ if $x=0$.

(ii) If $u_{1}^{\varepsilon}$ and $\nabla_{x} u_{0}^{\varepsilon}$ converge strongly in $L^{2}$ near $x \in \mathbb{R}^{d}$, then $\sigma_{0, \pm}=0$ a.e. in a neighborhood of $x$.

Proof. Suppose $y=(t, x) \in \Omega^{*}$. If $\sigma_{+}=\sigma_{-}=0$ near $y$, then $v_{ \pm}^{\varepsilon}=$ $\frac{1}{2}\left(\partial_{t} \mp \partial_{r}\right) u^{\varepsilon}$ converge strongly in $L^{2}$ near $y$. By (2.2.3), we know that $\Gamma_{k, l} u^{\varepsilon}$ also converge strongly. Writing

$$
\partial_{t} u^{\varepsilon}=v_{+}^{\varepsilon}+v_{-}^{\varepsilon}, \quad \partial_{x_{j}} u^{\varepsilon}=\frac{x_{j}}{|x|}\left(v_{-}^{\varepsilon}-v_{+}^{\varepsilon}\right)+\sum \frac{x_{k}}{|x|^{2}} \Gamma_{j, k} u^{\varepsilon}
$$

we conclude that $\nabla_{t, x} u^{\varepsilon}$ converge strongly.

If $y \in \Omega$ belongs to the time axis $\mathscr{A}:=\{x=0\}$, and if $\sigma_{+}=\sigma_{-}=0$ a.e. on the neighborhood $\omega$ of $y$, the previous step implies strong convergence 
of $\nabla_{t, x} u^{\varepsilon}$ in $L_{\text {loc }}^{2}(\omega \backslash \mathscr{A})$. Together with uniform $L^{2}$ estimates, this implies strong convergence in $L^{q}(\omega)$, for $q<2$.

Conversely, strong convergence for $u_{1}^{\varepsilon}$ and $\nabla_{x} u_{0}^{\varepsilon}$ implies strong convergence of $v_{0, \pm}^{\varepsilon}$ and thus the vanishing of $\sigma_{0, \pm}$.

Theorem 2.3.4 immediately follows from this lemma, Theorem 2.3.2 and Proposition 3.4.1.

\section{Proof of the GeOMETRIC OPTICS RESUlts}

In this section we show that if the initial data have profiles in the sense of Assumption 2.4.1, then the asymptotic behaviour of the solution is described with profiles and phases as indicated in Theorem 2.4.5. This allows one to follow in detail the absorption of singularities at the origin.

The proof resembles the analysis of the previous section in strategy. In analogy to the fact that one can associate weak limits and Young measures to bounded families, we will associate weak profiles and two scale Young measures which corresponds to enlarging the class of test functions to include oscillatory functions $a(y) e^{-i \alpha \chi(y) / \varepsilon}$. Then transport equations and inequalities are used to analyse the profiles and measures.

4.1. Profiles in the weak sense. Suppose that $\mathscr{O} \subset \mathbb{R}^{n}$ is open. The weak limit of a bounded subsequence $u^{\varepsilon}$ in $L^{p}(\mathscr{O})$ describes the action of $u^{\varepsilon}$ on smooth test functions. One can be more specific if one has in advance more information on the oscillations of the $u^{\varepsilon}$. In our case, we are particularly interested in functions which, at least formally, oscillate with phases

$$
\alpha \cdot \chi(y) / \varepsilon:=\sum \alpha_{j} \chi_{j}(y) / \varepsilon, \quad \alpha \in 2 \pi \mathbb{Z}^{m},
$$

where $\chi(y):=\left\{\chi_{1}(y), \ldots, \chi_{m}(y)\right\}$ is a given bounded $\mathbb{R}^{m}$-valued function.

To detect such behaviour, it is natural to test $u^{\varepsilon}$ against functions

$$
a(y) e^{-i \alpha \chi(y) / \varepsilon}, \quad a \in L^{p^{\prime}}(\mathscr{O}) .
$$

If $u^{\varepsilon}$ is bounded in $L^{p}(\mathscr{O}), 1<p \leq+\infty$, one can extract a subsequence such that, for all $\alpha \in 2 \pi \mathbb{Z}^{m}, e^{-i \alpha \chi(y) / \varepsilon} u^{\varepsilon}(y)$ is weakly convergent in $L^{p}(\mathscr{O})$ to a function $u_{\alpha}$ with

$$
\forall \alpha \in \mathbb{Z}^{m}:\left\|u_{\alpha}\right\|_{L^{p}} \leq \lim \sup \left\|u^{\varepsilon}\right\|_{L^{p}}
$$

In that case,

$$
\mathscr{U}(y, \theta):=\sum u_{\alpha}(y) e^{i \alpha \cdot \theta} \in \mathscr{D}^{\prime}\left(\mathscr{O} \times \mathbb{T}^{m}\right)
$$

and one has for any $\mathscr{A} \in C_{o}^{\infty}\left(\mathscr{O} \times \mathbb{T}^{m}\right)$

$$
\int u^{\varepsilon}(y) \mathscr{A}(y, \chi(y) / \varepsilon) d y \rightarrow\langle\mathscr{U}, \mathscr{A}\rangle .
$$

For a given subsequence there is at most one distribution $\mathscr{U}$ satisfying (4.1.5). Such a $\mathscr{U}$ will be called the weak profile of the subsequence.

For convenience, the period is chosen to be equal to 1 , so that $\theta$ varies in $\mathbb{T}^{m}, \mathbb{T}:=\mathbb{R} / \mathbb{Z} . \mathbb{T}^{m}$ is equipped with its invariant measure $d \theta$, of total mass 1. The dual of $\mathbb{T}^{m}$ is $2 \pi \mathbb{Z}^{m}$.

Formula (4.1.4) defines $\mathscr{U}$, as an $L^{p}(\mathscr{O})$ valued distribution on $\mathbb{T}^{m}$. With additional assumptions on $\chi$, we will prove that $\mathscr{U} \in L^{p}\left(\mathscr{O} \times \mathbb{T}^{m}\right)$. 
Assumption 4.1.1. The $\chi_{k}$ are $C^{1}$ on $\mathscr{O}$ and for all $\alpha \in 2 \pi \mathbb{Z}^{m} \backslash\{0\}, d(\alpha \cdot \chi) \neq 0$ almost everywhere on $\mathscr{O}$.

Lemma 4.1.2. Let

$$
\mathscr{A}(y, \theta):=\sum_{|\alpha| \leq k} a_{\alpha}(y) e^{i \alpha \cdot \theta}
$$

be a trigonometric polynomial with coefficients $a_{\alpha} \in L^{1}(\Omega)$. Then

$$
\int \mathscr{A}(y, \chi(y) / \varepsilon) d y \rightarrow \int \mathscr{A}(y, \theta) d y d \theta
$$

Proof. It is sufficient to prove that

$$
\lim _{\varepsilon \rightarrow 0} \int a_{\alpha}(y) e^{i \alpha \cdot \chi(y) / \varepsilon} d x= \begin{cases}0 & \text { if } \alpha \neq 0 \\ \int a_{0}(y) d y & \text { if } \alpha=0\end{cases}
$$

Assumption 4.1.1 and integration by parts show this is true if $a_{\alpha} \in C_{o}^{\infty}\left(\mathscr{O}_{\alpha}\right)$ where $\mathscr{O}_{\alpha}:=\{y \in \mathscr{O} \mid \alpha \cdot d \chi(y) \neq 0\}$. Since $C_{o}^{\infty}\left(\mathscr{O}_{\alpha}\right)$ is dense in $L^{1}(\mathscr{O})$, formula (4.1.8) extends to all $a_{\alpha} \in L^{1}(\mathscr{O})$.

Lemma 4.1.3. Formula (4.1.7) is valid for all $\mathscr{A} \in C_{o}^{0}\left(\mathscr{O} \times \mathbb{T}^{m}\right)$. Moreover, if $\mathscr{A} \in C_{o}^{0}\left(\mathscr{O} \times \mathbb{T}^{m}\right)$ or if the coefficients $a_{\alpha}$ of a trigonometric polynomial (4.1.6) belong to $L^{p}(\mathscr{O}), 1 \leq p<\infty$, then

$$
\int|\mathscr{A}(y, \chi(y) / \varepsilon)|^{p} d y \rightarrow \int|\mathscr{A}(y, \theta)|^{p} d y d \theta .
$$

Proof. To prove the first statement, choose trigonometric polynomials $\mathscr{A}_{n}$ with $C_{o}^{0}(\mathscr{O})$ coefficients such that $\mathscr{A}_{n} \rightarrow \mathscr{A}$ in $L^{\infty}\left(\mathscr{O} \times \mathbb{T}^{m}\right)$. Then

$$
\int \mathscr{A}_{n}(y, \theta) d y d \theta \rightarrow \int \mathscr{A}(y, \theta) d y d \theta
$$

and

$$
\int \mathscr{A}_{n}(y, \chi(y) / \varepsilon) d y \rightarrow \int \mathscr{A}(y, \chi(y) / \varepsilon) d y
$$

as $n \rightarrow+\infty$, uniformly in $\varepsilon$. This together with (4.1.7) applied to $\mathscr{A}_{n}$ yields (4.1.7) for $\mathscr{A}$.

Next, (4.1.7) applied to $|\mathscr{A}|^{p}$ is (4.1.9). Finally, if $\mathscr{A}$ is a trigonometric polynomial with $L^{p}(\mathscr{O})$ coefficients, one can approximate these coefficients in $L^{p}$ by $C_{o}^{0}(\mathscr{O})$ functions. Applying (4.1.9) to the approximations in $C_{o}^{0}\left(\mathscr{O} \times \mathbb{T}^{d}\right)$ and passing to the limit yield the result.

The existence of $L^{2}$ profiles with linear phases has already been used by several authors (see $[\mathrm{N}],[\mathrm{E}],[\mathrm{ES}])$. The $L^{p}$ case with general phases satisfying Assumption 4.1.1 is similar.

Proposition 4.1.4. Suppose that $u^{\varepsilon}$ is a bounded sequence in $L^{p}(\mathscr{O}), 1<p \leq$ $+\infty$, and $\mathscr{U}$ is a weak profile for a subsequence. If Assumption 4.1 .1 is satisfied, then $\mathscr{U} \in L^{p}\left(\mathscr{O} \times \mathbb{T}^{m}\right)$ and (4.1.5) extends to all $\mathscr{A} \in C_{o}^{0}\left(\mathscr{O} \times \mathbb{T}^{m}\right)$, that is

$$
\int u^{\varepsilon}(y) \mathscr{A}(y, \chi(y) / \varepsilon) d y \rightarrow \int \mathscr{U}(y, \theta) \mathscr{A}(y, \theta) d y d \theta
$$

Proof. For $\mathscr{A} \in C_{o}^{\infty}\left(\mathscr{O} \times \mathbb{T}^{m}\right)$, estimate

$$
\begin{aligned}
|\langle\mathscr{U}, \mathscr{A}\rangle| & =\lim \left|\int u^{\varepsilon}(y) \mathscr{A}(y, \chi(y) / \varepsilon) d y\right| \\
& \leq \lim \sup \left\|u^{\varepsilon}\right\|_{L^{p}(\mathscr{O})}\|\mathscr{A}(\cdot, \chi(\cdot) / \varepsilon)\|_{L^{p^{\prime}}(\mathscr{O})} \leq C\|\mathscr{A}(\cdot, \cdot)\|_{L^{p^{\prime}}\left(\mathscr{O} \times \mathbb{T}^{m}\right)}
\end{aligned}
$$


the last inequality using Lemma 3.1.4. It follows that $\mathscr{U}$ belongs to $L^{p}\left(\mathscr{O} \times \mathbb{T}^{m}\right)$.

Approximating $\mathscr{A} \in C_{o}^{0}\left(\mathscr{O} \times \mathbb{T}^{m}\right)$ by elements of $C_{o}^{\infty}\left(\mathscr{O} \times \mathbb{T}^{m}\right)$ proves the final assertion of the proposition.

Example 4.1.5. If $\mathscr{A} \in C_{o}^{0}\left(\mathscr{O} \times \mathbb{T}^{m}\right)$ and $u^{\varepsilon}(y):=\mathscr{A}(y, \chi(y) / \varepsilon)$, then $\mathscr{U}(y, \theta)$ $=\mathscr{A}(y, \theta)$.

4.2. Profiles in the strong sense. It is tempting to think that $\mathscr{U}(y, \chi(y) / \varepsilon)$ is an approximation to $u^{\varepsilon}$. In general, this is not the case. This is analogous to the fact that, in general, weak limits are not good approximations. Another difficulty arises here. If $\mathscr{U}$ is not sufficiently smooth, the substitution $\theta=\chi(y) / \varepsilon$ in $\mathscr{U}(y, \theta)$ does not make sense. Even if $\mathscr{U}$ is smooth, $\mathscr{U}(y, \chi(y) / \varepsilon)$ is not necessarily a good approximation of $u^{\varepsilon}$ because $\mathscr{U}$ does not see the oscillations of wavelength of order $\neq \varepsilon$, nor the oscillations with phases $\psi$ which are linearly independent of the given $\chi$. This is seen in the following two examples.

(i) Suppose that $m=1$ and $\chi^{\prime} \neq 0$. Then, if $a \in L^{p}(\mathscr{O}), u^{\varepsilon}(y)=$ $a(y) e^{i \chi(y) / \varepsilon^{2}}$ has profile $\mathscr{U}=0$. Similarly, $v^{\varepsilon}(y)=a(y) e^{i \chi(y) / \sqrt{\varepsilon}}$ has profile $\mathscr{V}=0$.

(ii) Suppose that $m=1, \chi^{\prime} \neq 0$ and $\alpha \in L^{p}(\mathscr{O})$. Suppose that $\psi \in C^{\infty}$ is such that $d(\chi-\psi) \neq 0$ a.e. Then $u^{\varepsilon}(y)=a(y) e^{i \psi(y) / \varepsilon}$ has profile $\mathscr{U}=0$.

The aim of this section is to study the strong approximation of $u^{\varepsilon}(y)$ by $\mathscr{U}(y, \chi(y) / \varepsilon)$ in the sense that

$$
\left\|u^{\varepsilon}(\cdot)-\mathscr{U}(\cdot, \chi(\cdot) / \varepsilon)\right\|_{L^{p}(\mathscr{O})} \rightarrow 0 \quad \text { as } \varepsilon \rightarrow 0 .
$$

When $\mathscr{U}$ is not smooth, for instance $\mathscr{U} \in L^{2}\left(\mathscr{O} \times \mathbb{T}^{m}\right)$, the expression $\mathscr{U}(y, \chi(y) / \varepsilon)$ requires care, since for each $\varepsilon$ the set $\{(y, \chi(y) / \varepsilon)\}$ is a set of measure zero in $\mathscr{O} \times \mathbb{T}^{m}$. Nevertheless, one can give an asymptotic sense to the substitution, and the definition of asymptotic equivalence $u^{\varepsilon}(y) \sim \mathscr{U}(y, \chi(y) / \varepsilon)$ given by (4.2.1) when $\mathscr{U}$ is continuous can be extended to $\mathscr{U} \in L^{p}$.

We suppose that Assumption 4.1.1 is satisfied.

Definition 4.2.1. Suppose that $\mathscr{U} \in L^{p}\left(\mathscr{O} \times \mathbb{T}^{m}\right), 1 \leq p \leq+\infty$, and $\left\{u^{\varepsilon}\right\}_{\varepsilon \in] 0,1]}$ $\subset L^{p}(\mathscr{O})$. We say that

$$
u^{\varepsilon}(y) \sim \mathscr{U}(y, \chi(y) / \varepsilon) \text { in } L^{p}(\mathscr{O})
$$

when there exist a sequence of trigonometric polynomials $\mathscr{U}_{l}$ with coefficients in $L^{p}(\mathscr{O})$ and sequences of real numbers $\varepsilon_{l}>0$ and $\delta_{l}>0$, with $\delta_{l} \rightarrow 0$, such that for all $l$

$$
\begin{gathered}
\left\|\mathscr{U}-\mathscr{U}_{l}\right\|_{L^{p}\left(\mathscr{O} \times \mathbb{T}^{m}\right)} \leq \delta_{l}, \\
\left.\forall \varepsilon \in] 0, \varepsilon_{l}\right]:\left\|u^{\varepsilon}(\cdot)-\mathscr{U}_{l}(\cdot, \chi(\cdot) / \varepsilon)\right\|_{L^{p}(\mathscr{O})} \leq \delta_{l} .
\end{gathered}
$$

Remark 4.2.2. Because the Fourier coefficients of $\mathscr{U}_{0}$ are in $L^{p}(\mathscr{O}), u_{0}^{\varepsilon}(y):=$ $\mathscr{U}_{0}(y, \chi(y) / \varepsilon) \in L^{p}(\mathscr{O})$, so that $u^{\varepsilon}$ is necessarily bounded in $L^{p}(\mathscr{O})$ (for $\varepsilon \leq$ $\left.\varepsilon_{0}\right)$.

Proposition 4.2.3. (i) Let $\mathscr{U} \in L^{p}\left(\mathscr{O} \times \mathbb{T}^{m}\right)$, with $1 \leq p<+\infty$. Then there exists $u^{\varepsilon} \sim \mathscr{U}(y, \chi(y) / \varepsilon)$ in $L^{p}$. Moreover, another sequence $\tilde{u}^{\varepsilon}$ is $\sim \mathscr{U}(y, \chi(y) / \varepsilon)$ if and only if $u^{\varepsilon}-\tilde{u}^{\varepsilon} \rightarrow 0$ strongly in $L^{p}(\mathscr{O})$ as $\varepsilon \rightarrow 0$.

(ii) When $\mathscr{U} \in C_{o}^{0}\left(\mathscr{O} \times \mathbb{T}^{d}\right)$, (4.2.2) holds if and only if $u^{\varepsilon}(y)-\mathscr{U}(y, \chi(y) / \varepsilon)$ $\rightarrow 0$ in $L^{p}(\mathscr{O})$. 
(iii) Let $\mathscr{U} \in L^{p}\left(\mathscr{O} \times \mathbb{T}^{m}\right)$ be such that $\partial_{\theta} \mathscr{U} \in L^{p}\left(\mathscr{O} \times \mathbb{T}^{m}\right)$. Then the substitution $\mathscr{U}(y, \chi(y) / \varepsilon)$ makes sense and (4.2.2) holds if and only if $u^{\varepsilon}(y)-$ $\mathscr{U}(y, \chi(y) / \varepsilon) \rightarrow 0$ in $L^{p}(\mathscr{O})$.

Proof. (a) Consider a family of trigonometric polynomials $\mathscr{U}_{l}$ such that $\mathscr{U}_{l} \rightarrow$ $\mathscr{U}$ in $L^{p}\left(\mathscr{O} \times \mathbb{T}^{m}\right)$. Let $u_{l}^{\varepsilon}(y):=\mathscr{U}_{l}(y, \chi(y) / \varepsilon)$. Then Lemma 4.1.3 implies that, for any $k$ and $l$,

$$
\lim _{\varepsilon \rightarrow 0}\left\|u_{k}^{\varepsilon}-u_{l}^{\varepsilon}\right\|_{L^{p}(\mathscr{O})}=\left\|\mathscr{U}_{k}-\mathscr{U}_{l}\right\|_{L^{p}\left(\mathscr{O} \times \mathbb{T}^{m}\right)} .
$$

Let $\delta_{l}:=\sup \left\{2\left\|\mathscr{U}_{k}-\mathscr{U}_{l}\right\|_{L^{p}}, k \geq l\right\}$. Then $\delta_{l} \rightarrow 0$ and (4.2.3) is satisfied. Next, because of (4.2.5), we see that for every $k \geq l$ there exists $\varepsilon_{k, l}>0$ such that

$$
\left.\forall \varepsilon \in] 0, \varepsilon_{k, l}\right]:\left\|u_{k}^{\varepsilon}-u_{l}^{\varepsilon}\right\|_{L^{p}} \leq \delta_{l} .
$$

Let $\varepsilon_{k}>0$ be the smallest value of the $\varepsilon_{k, l}$ for $0 \leq l \leq k$. Then

$$
\left.\forall l \leq k, \forall \varepsilon \in] 0, \varepsilon_{k}\right]:\left\|u_{k}^{\varepsilon}-u_{l}^{\varepsilon}\right\|_{L^{p}} \leq \delta_{l} .
$$

There is no restriction in assuming that $\varepsilon_{k}$ decreases with $k$ and that $\varepsilon_{k} \rightarrow 0$ as $k \rightarrow+\infty$. With these notations, let $k(\varepsilon):=\operatorname{Max}\left\{k \mid \varepsilon \leq \varepsilon_{k}\right\}$. Then

$$
\forall \varepsilon \leq \varepsilon_{0}, \varepsilon \leq \varepsilon_{k(\varepsilon)} \text { and } k(\varepsilon) \rightarrow+\infty \quad \text { as } \varepsilon \rightarrow 0 \text {. }
$$

Define $u^{\varepsilon}:=u_{k(\varepsilon)}^{\varepsilon}$. Then for $\varepsilon \leq \varepsilon_{l}$, one has $l \leq k(\varepsilon)$. Thus (4.2.6) implies that the second condition (4.2.4) is also satisfied.

(b) Suppose that $u^{\varepsilon} \sim \mathscr{U}(y, \chi(y) / \varepsilon) \sim \tilde{u}^{\varepsilon}$. Call $\mathscr{U}_{l}, \tilde{\mathscr{U}}_{l}, \varepsilon_{l}, \tilde{\varepsilon}_{l}, \delta_{l}, \tilde{\delta}_{l}$ the corresponding sequences. Lemma 4.1.3 implies that

$$
\limsup _{\varepsilon \rightarrow 0}\left\|u_{k}^{\varepsilon}-\tilde{u}_{l}^{\varepsilon}\right\|_{L^{p}} \leq\left\|\mathscr{U}_{k}-\tilde{\mathscr{U}}_{l}\right\|_{L^{p}}
$$

Thus, for any $l$, there is $\varepsilon_{l}^{\prime} \leq \min \left\{\varepsilon_{k}, \tilde{\varepsilon}_{l}\right\}, \varepsilon_{l}^{\prime}>0$, such that for $\varepsilon \leq \varepsilon_{l}^{\prime}$ one has

$$
\left\|u^{\varepsilon}-\tilde{u}^{\varepsilon}\right\|_{L^{p}} \leq 3\left(\delta_{l}+\tilde{\delta}_{l}\right)
$$

which implies that $u^{\varepsilon}-\tilde{u}^{\varepsilon} \rightarrow 0$ in $L^{p}$.

The converse statement is obvious.

(c) When $\mathscr{U} \in C_{o}^{0}\left(\mathscr{O} \times \mathbb{T}^{d}\right)$ the substitution $u^{\varepsilon}(y)=\mathscr{U}(y, \chi(y) / \varepsilon)$ makes sense and the claim is clear.

(d) Suppose that $\mathscr{U} \in L^{p}\left(\mathscr{O} \times \mathbb{T}^{m}\right)$ is such that $\partial_{\theta} \mathscr{U} \in L^{p}\left(\mathscr{O} \times \mathbb{T}^{m}\right)$. Then $\theta \rightarrow \mathscr{U}(y, \theta+\chi(y) / \varepsilon)$ is continuous from $\mathbb{T}$ to $L^{p}(\mathscr{O})$. The substitution $\mathscr{U}(y, \theta+\chi(y) / \varepsilon)$ is defined as the trace at $\theta=0$ of this mapping.

The Sobolev imbedding $W^{1,1}(\mathbb{T}) \subset L^{\infty}(\mathbb{T})$ yields the estimate (4.2.8)

$$
|\mathscr{U}(y, \theta)| \leq C a(y) \quad \text { with } a(y):=\|\mathscr{U}(y, \cdot)\|_{L^{p}(\mathbb{T})}+\left\|\partial_{\theta} \mathscr{U}(y, \cdot)\right\|_{L^{p}(\mathbb{T})} \in L^{p}(\mathscr{O}) .
$$

Choose a sequence $\mathscr{U}_{k}$ of trigonometric polynomials such that $\mathscr{U}_{k} \rightarrow \mathscr{U}$ and $\partial_{\theta} \mathscr{U}_{k} \rightarrow \partial_{\theta} \mathscr{U}$ in $L^{p}(\mathscr{O} \times \mathbb{T})$. (4.2.8) implies that

$$
\left|\mathscr{U}(y, \chi(y) / \varepsilon)-\mathscr{U}_{k}(y, \chi(y) / \varepsilon)\right| \leq C a_{k}(y)
$$

where $a_{k} \rightarrow 0$ in $L^{p}(\mathscr{O})$. Part (iii) of Proposition 4.2.3 follows.

Lemma 4.1.2 has the following extension. 
Lemma 4.2.4. Suppose that $\mathscr{U} \in L^{1}\left(\mathscr{O} \times \mathbb{T}^{m}\right)$ and $u^{\varepsilon} \sim \mathscr{U}(y, \chi(y) / \varepsilon)$. Then

$$
\int u^{\varepsilon}(y) d y \rightarrow \int \mathscr{U}(y, \theta) d y d \theta .
$$

Proof. Approximate $\mathscr{U}$ by trigonometric polynomials $\mathscr{U}_{l}$ and $u^{\varepsilon}$ by the $u_{l}^{\varepsilon}(y)$ $:=\mathscr{U}_{l}(y, \chi(y) / \varepsilon)$. Applying Lemma 4.1.2 to the $\mathscr{U}_{l}$ yields the result.

For later use, we give a variant of Proposition 4.2.3.

Proposition 4.2.5. Let $\left\{X_{j}\right\}$ be smooth vector fields on $\mathscr{O}$ such that, for all $j$ and $k, X_{j} \chi_{k}=0$. Suppose that $\mathscr{U} \in L^{2}\left(\mathscr{O} \times \mathbb{T}^{m}\right)$ is such that, for all $j, \mathscr{U}_{j}(y, \theta):=$ $X_{j}\left(y, \partial_{y}\right) \mathscr{U}(y, \theta) \in L^{\alpha_{j}}\left(\mathscr{O} \times \mathbb{T}^{m}\right), \alpha_{j} \in[1,2]$. Then there is $u^{\varepsilon} \in L^{2}(\mathscr{O})$ such that $u^{\varepsilon} \sim \mathscr{U}(y, \chi(y) / \varepsilon), X_{j} u^{\varepsilon} \in L^{\alpha_{j}}(\mathscr{O})$ and $X_{j} u^{\varepsilon} \sim \mathscr{U}_{j}(y, \chi(y) / \varepsilon)$ in $L^{\alpha_{j}}(\mathscr{O})$.

Proof. There is a sequence of trigonometric polynomial $\mathscr{U}_{\nu}$ such that

$$
\mathscr{U}_{\nu} \rightarrow \mathscr{U} \text { in } L^{2}\left(\mathscr{O} \times \mathbb{T}^{m}\right) \text { and } X_{j} \mathscr{U}_{\nu} \rightarrow X_{j} \mathscr{U} \quad \text { in } L^{\alpha_{j}}\left(\mathscr{O} \times \mathbb{T}^{m}\right) \text {. }
$$

As in Definition 4.1.4, let $u_{l}^{\varepsilon}(y):=\mathscr{U}_{l}(y, \chi(y) / \varepsilon)$. Then

$$
X_{j} u_{l}^{\varepsilon}(y):=X_{j} \mathscr{U}_{l}(y, \chi(y) / \varepsilon),
$$

is implied by the equations $X_{j} \chi_{k}=0$. Part (a) of the proof of Proposition 4.1.6 can be followed jointly for $u_{l}^{\varepsilon}(y)$ and the $X_{j} u_{l}^{\varepsilon}(y)$ to define a $u^{\varepsilon}:=u_{k(\varepsilon)}^{\varepsilon} \sim$ $\mathscr{U}(y, \chi(y) / \varepsilon)$ such that $X_{j} u^{\varepsilon}=X_{j} u_{k(\varepsilon)}^{\varepsilon} \sim X_{j} \mathscr{U}(y, \chi(y) / \varepsilon)$.

Proposition 4.1.4 has the following extension.

Proposition 4.2.6. Suppose that $u^{\varepsilon}$ is a bounded sequence in $L^{p}(\mathscr{O}), 1<p \leq$ $+\infty$, with weak profile $\mathscr{U}, \mathscr{A} \in L^{p^{\prime}}\left(\Omega \times \mathbb{T}^{m}\right)$ and $a^{\varepsilon} \sim \mathscr{A}(x, \chi(x) / \varepsilon)$. Then

$$
\int u^{\varepsilon}(y) a^{\varepsilon}(y) d y \rightarrow \int \mathscr{U}(y, \theta) \mathscr{A}(y, \theta) d y d \theta .
$$

Proof. Let $\mathscr{A}_{l}$ be approximations of $\mathscr{A}$ by trigonometric polynomials. Then

$$
\int \mathscr{U}\left\{\mathscr{A}-\mathscr{A}_{l}\right\} d y d \theta \text { and } \int u^{\varepsilon}\left\{a^{\varepsilon}-a_{l}^{\varepsilon}\right\} d y
$$

are arbitrarily small for $l$ large enough and $\varepsilon \leq \varepsilon_{l}$. On the other hand, the convergence (4.2.11) is known for the trigonometric polynomials $\mathscr{A}_{l}$. Proposition 4.2.6 follows.

Corollary 4.2.7. Let $\mathscr{U} \in L^{p}\left(\mathscr{O} \times \mathbb{R}^{m}\right)$ and let $u^{\varepsilon} \sim \mathscr{U}(y, \chi(y) / \varepsilon)$. Then $\mathscr{U}$ is the weak profile for the sequence $u^{\varepsilon}$.

Proof. Let $\mathscr{A} \in C_{o}^{0}\left(\mathscr{O} \times \mathbb{R}^{m}\right)$ and let $a^{\varepsilon}(x):=\mathscr{A}(y, \chi(y) / \varepsilon)$. Then $\mathscr{U} \mathscr{A} \in$ $L^{p}\left(\mathscr{O} \times \mathbb{R}^{m}\right)$ and one easily checks that $u^{\varepsilon} a^{\varepsilon} \sim(\mathscr{L} \mathscr{A})(y, \chi(y) / \varepsilon)$. Therefore the convergence (4.2.1) follows from Lemma 4.1.7.

4.3. Two-scale Young measures. The definition of Young measures has already been extended to two-scale analysis (see [E], [E S]). Here we collect definitions and results which will be needed. They are very similar to results of section 3.1, and we will not repeat the proofs. The framework is as in subsection 4.1: we are given a set of phases $\chi_{k}, k=1, \ldots, m$, which satisfy Assumption 4.1.1. 
Proposition 4.3.1. Given a bounded family $\left\{u^{\varepsilon}\right\} \subset L^{p}(\mathscr{O})$ of $\mathbb{R}^{N}$-valued functions, there is a subsequence and a measurable family of probability measures on $\mathbb{R}^{N},\left\{\mu(y, \theta, \cdot),(y, \theta) \in \mathscr{O} \times \mathbb{T}^{m}\right\}$, such that for any continuous function $f(\lambda)$ on $\mathbb{R}^{N}$ which tends to 0 at infinity and for all $\mathscr{A} \in C_{o}^{0}\left(\Omega \times \mathbb{T}^{m}\right)$

$$
\int \mathscr{A}(y, \chi(y) / \varepsilon) f\left(u^{\varepsilon}(y)\right) d y \rightarrow \iint \mathscr{A}(y, \theta) f(\lambda) \mu(y, \theta, d \lambda) d y d \theta
$$

The double integral notation is used to make clear the distinction between the variables $\lambda$ and $(y, \theta)$ which do not play similar roles.

The measure $\mu:=\mu(y, \theta, d \lambda) d y d \theta$ on $\mathscr{O} \times \mathbb{T}^{m} \times \mathbb{R}^{N}$ is called the two-scale Young measure of the subsequence $u^{\varepsilon}$. We introduce the following convenient terminology.

Definition 4.3.2. A bounded family $u^{\varepsilon}$ in $L^{p}$ is pure when there exists a measure $\mu$ satisfying (4.3.1).

Remark 4.3.3. If $u^{\varepsilon}$ is pure in the sense of Definition 4.3.2, it is automatically pure in the sense of Definition 3.1.2 and the measures $\mu(y, \cdot)$ and $\mu(y, \theta, \cdot)$ are related by the relation

$$
\iint a(y) f(\lambda) \mu(y, d \lambda) d y=\iint a(y) f(\lambda) \mu(y, \theta, d \lambda) d y d \theta .
$$

Formula (4.3.2) shows that $\mu(y, \theta, \cdot)$ gives finer information than $\mu(y, \cdot)$ so that many properties of the latter can be deduced from corresponding properties of the former. However, the existence of $\mu(y, \cdot)$ for a subsequence does not imply the existence of $\mu(y, \theta, \cdot)$ and the definition of $\mu(y, \theta, \cdot)$ depends on the choice of the phase functions $\chi$.

Proposition 4.3.4. Suppose that $u^{\varepsilon}$ is a pure bounded family in $L^{p}(\mathscr{O})$ with two-scale Young measure $\mu$. Then $|\lambda| \in L^{p}\left(\mathscr{O} \times \mathbb{R}^{N}, \mu\right)$ and

$$
\iint|\lambda|^{p} \mu(y, \theta, d \lambda) d y d \theta \leq \liminf \left\|u^{\varepsilon}\right\|_{L^{p}(\mathscr{O})}^{p}
$$

Proposition 4.3.5. Suppose that $u^{\varepsilon}$ is a pure bounded family in $L^{p}(\mathscr{O})$ with twoscale Young measure $\mu, F(\lambda, \tau)$ is a continuous function such that $|F(\lambda, \tau)|$ $=o\left(|\lambda|^{p}+|\tau|^{p}\right)$ as $|\lambda|+|\tau| \rightarrow+\infty, a \in L^{p}\left(\mathscr{O} \times T^{m}\right)$, and $a^{\varepsilon} \sim a(y, \chi(y) / \varepsilon)$ in $L^{p}(\mathscr{O})$. Then for all $\varphi \in C_{o}^{0}(\overline{\mathscr{O}})$,

$$
\int \varphi(y) F\left(u^{\varepsilon}(y), a^{\varepsilon}(y)\right) d y \rightarrow \iint \varphi(y) F(\lambda, \mathscr{A}(y, \theta)) \mu(y, \theta, d \lambda) d y d \theta .
$$

Moreover, if $|F(\lambda, \tau)|=O\left(|\lambda|^{q}+|\tau|^{q}\right)$ as $|\lambda|+|\tau| \rightarrow+\infty$, with $q<p$, then the convergence (4.3.4) extends to all $\varphi \in L_{\text {comp }}^{r}(\overline{\mathscr{O}})$, with $1 / r+q / p=1$.

Proposition 4.3.5 implies that the convergence (4.3.1) extends to all $f \in$ $C^{0}\left(\mathbb{R}^{N}\right)$ such that $f(\lambda)=o\left(|\lambda|^{p}\right)$ as $|\lambda| \rightarrow+\infty$.

Remark 4.3.6. In particular, Proposition 4.3.5 implies that the convergence (4.3.1) holds for $f(\lambda)=\lambda$, provided that $u^{\varepsilon}$ is a pure bounded family in $L^{p}(\mathscr{O})$ with $p>1$. Comparing (4.3.1) and (4.1.10), we see that, when $p>1$, the weak profile $\mathscr{U}$ associated to the pure family $u^{\varepsilon}$ is the expectation in $\lambda$ of the measure $\mu$ :

$$
\mathscr{U}(y, \theta)=\int \lambda \mu(y, \theta, d \lambda), \quad \text { a.e. }(y, \theta) \in \mathscr{O} \times \mathbb{T}^{m} .
$$


More generally, for continuous $f(\lambda)=o\left(\lambda^{p}\right)$ at infinity,

$$
\mathscr{F}(y, \theta)=\int f(\lambda) \mu(y, \theta, d \lambda), \quad \text { a.e. }(y, \theta) \in \mathscr{O} \times \mathbb{T}^{d},
$$

is the weak profile of the sequence $f\left(u^{\varepsilon}\right)$.

Proposition 4.3.5 admits the following corollary.

Corollary 4.3.7. Suppose that $u^{\varepsilon}$ is a pure bounded family in $L^{p}(\mathscr{O})$ with twoscale Young measure $\mu, \mathscr{A} \in L^{p}\left(\mathscr{O} \times \mathbb{T}^{m}\right)$, and $a^{\varepsilon} \sim \mathscr{A}(y, \chi(y) / \varepsilon)$ in $L^{p}(\mathscr{O})$. Then $v^{\varepsilon}:=u^{\varepsilon}-a^{\varepsilon}$ is a pure family in $L^{p}(\mathscr{O})$, and the associated measure $\nu$ is given by

$$
\begin{aligned}
\iint & \Phi(y, \theta) f(\lambda) \nu(y, \theta, d \lambda) d y d \theta \\
& =\iint \Phi(y, \theta) f(\lambda-\mathscr{A}(y, \theta)) \mu(y, \theta, d \lambda) d y d \theta
\end{aligned}
$$

for all $\Phi \in C_{o}^{\infty}\left(\mathscr{O} \times \mathbb{T}^{m}\right)$ and all $f \in C_{o}^{0}\left(\mathbb{R}^{N}\right)$.

Proposition 4.3.8. Suppose that $\mathscr{U} \in L^{p}\left(\mathscr{O} \times \mathbb{T}^{m}\right)$ and $u^{\varepsilon} \sim \mathscr{U}(y, \chi(y) / \varepsilon)$. Then the family $u^{\varepsilon}$ is pure in the sense of Definition 4.3 .2 and the associated two-scale Young measure is given by

$$
\iint \mathscr{A}(y, \theta) f(\lambda) \mu(y, \theta, d \lambda) d y d \theta=\iint \mathscr{A}(y, \theta) f(\mathscr{U}(y, \theta)) d y d \theta .
$$

Proof. For $f \in C_{o}^{1}(\mathbb{R})$, or more generally $f \in C^{1}$ such that $|f(\lambda)|=o\left(|\lambda|^{p}\right)$, one checks that $f\left(u^{\varepsilon}\right) \sim f(\mathscr{U}(y, \chi(y) / \varepsilon))$ in $L^{1}$. Thus $f(\mathscr{U})$ is the profile of $f\left(u^{\varepsilon}\right)$. Next apply Proposition 4.2.6 to show that

$$
\int f\left(u^{\varepsilon}(x)\right) \mathscr{A}(x, \chi(x) / \varepsilon) d x \rightarrow \iint f(\mathscr{U}(x, \theta)) \mathscr{A}(x, \theta) d x d \theta,
$$

proving Proposition 4.3.8.

Conversely, one has

Proposition 4.3.9. Suppose that $\mathscr{U} \in L^{p}\left(\mathscr{O} \times \mathbb{T}^{d}\right), p>1$, and $u^{\varepsilon}$ is a pure bounded family in $L^{p}(\mathscr{O})$ such that, for almost all $(y, \theta), \mu(y, \theta, d \lambda)$ is the Dirac measure at $\lambda=\mathscr{U}(y, \theta)$. Then $u^{\varepsilon} \sim \mathscr{U}(y, \chi(y) / \varepsilon)$ in $L_{\text {loc }}^{q}(\overline{\mathscr{O}})$ for all $q<p$. In particular, if $p \geq 2$, and if the variance

$$
\sigma(y, \theta):=\int|\lambda-\mathscr{U}(y, \theta)|^{2} \mu(y, \theta, d \lambda), \quad \text { a.e. }(y, \theta) \in \mathscr{O} \times \mathbb{T}^{m},
$$

vanishes on $\mathscr{O}$, then $u^{\varepsilon} \sim \mathscr{U}(y, \chi(y) / \varepsilon)$ in $L_{\text {loc }}^{q}(\overline{\mathscr{O}})$ for all $q<p$.

Proof. Choose $m^{\varepsilon} \sim \mathscr{U}(y, \chi(y) / \varepsilon)$ and let $v^{\varepsilon}:=u^{\varepsilon}-m^{\varepsilon}$. According to Corollary 4.3.6, the family $v^{\varepsilon}$ is pure and the associated measure $\mu(y, \theta, \cdot)$ is the Dirac mass at $\lambda=0$. Proposition 4.3.5 implies that

$$
\int \varphi(y)\left|v^{\varepsilon}(y)\right|^{q} d y \rightarrow \int \varphi(y)|\lambda|^{q} \mu(y, \theta, d \lambda) d y d \theta=0
$$

for all $q<p$ and all $\varphi \in L_{\text {comp }}^{r}(\overline{\mathscr{O}}), 1 / r+q / p=1$. This proves Proposition 4.3.9.

When the $u^{\varepsilon}$ satisfy an equation, the two-scale Young measures satisfy a related equation. 
Proposition 4.3.10. Suppose that $u^{\varepsilon}$ is a pure bounded sequence in $L^{p}(\mathscr{O})$ with two-scale measure $\mu$, and $X\left(y, \partial_{y}\right)$ is a smooth vector field on $\mathscr{O}$. Assume that $X u^{\varepsilon}$ is bounded in $L_{\text {loc }}^{1}(\mathscr{O})$. Then $\mathscr{X} \mu=0$ in the distribution sense where $\mathscr{X}\left(y, \partial_{\theta}\right)$ is the vector field on $\mathscr{O} \times \mathbb{T}^{m} \times \mathbb{R}_{\lambda}^{N}$ defined by

$$
\mathscr{X}\left(y, \partial_{\theta}\right):=\sum_{j=1}^{m}\left\{X \chi_{j}\right\}(y) \partial_{\theta_{j}} .
$$

Proof. Let $\mathscr{A}(y, \theta) \in C_{o}^{\infty}\left(\mathscr{O} \times \mathbb{T}^{m}\right)$. Introduce $\mathscr{B}(y, \theta):=\mathscr{X}\left(y, \partial_{\theta}\right) \mathscr{A}(y, \theta)$ and $\mathscr{C}(y, \theta):=X\left(y, \partial_{y}\right) \mathscr{A}(y, \theta)$. Let $a^{\varepsilon}(y):=\mathscr{A}(y, \chi(y) / \varepsilon), b^{\varepsilon}(y):=$ $\mathscr{B}(y, \chi(y) / \varepsilon)$ and $c^{\varepsilon}(y):=\mathscr{C}(y, \chi(y) / \varepsilon)$. Then

$$
b^{\varepsilon}=\varepsilon X a^{\varepsilon}-\varepsilon c^{\varepsilon} .
$$

Multiply (4.3.12) by $f\left(u^{\varepsilon}\right)$ and integrate over $\mathscr{O}$. Because $X f\left(u^{\varepsilon}\right)=f^{\prime}\left(u^{\varepsilon}\right) X u^{\varepsilon}$ is bounded in $L_{\text {loc }}^{1}$, we obtain

$$
\int b^{\varepsilon}(y) f\left(u^{\varepsilon}(y)\right) d y=O(\varepsilon) \rightarrow 0 .
$$

By definition of $\mu$, this means that

$$
\int\left\{\mathscr{X}\left(x, \partial_{\theta}\right) \mathscr{A}(x, \theta)\right\} f(\lambda) \mu(x, \theta, d \lambda) d x d \theta=0
$$

and therefore $\mathscr{X} \mu=0$ in the sense of distributions.

4.4. Independence of the Young measures. Let $u^{\varepsilon}$ be a family of solutions to (2.1.1) which satisfies the estimates (2.1.4), (2.1.5). With $\chi \in C^{\infty}(\mathbb{R})$, such that $\chi^{\prime} \neq 0$ a.e., $\chi^{\prime} \in L^{\infty}$, introduce the phases

$$
\chi_{ \pm}(t, x):=\chi(|x| \mp t)
$$

which are smooth on $\Omega^{*}:=\Omega \backslash\{x=0\}$, and satisfy Assumption 4.1.1 on $\Omega^{*}$.

We assume that a subsequence has been extracted so that

$$
\left\{\begin{array}{l}
\text { The convergences }(2.2 .1)-(2.2 .5) \text { hold. } \\
\text { The bounded families in } L^{2}\left(\Omega_{T}\right), v_{ \pm}^{\varepsilon} \text {, defined in (2.2.10) are pure. } \\
\text { The bounded family in } L^{p}(\Omega), \partial_{t} u^{\varepsilon}=v_{+}^{\varepsilon}+v_{-}^{\varepsilon} \text {, is pure. } \\
\text { The families of Cauchy data } v_{o, \pm}^{\varepsilon}(2.2 .13) \text { are pure }
\end{array}\right.
$$

Here, pure is taken in the sense of Definition 4.3.2, with the set of phases $\left\{\chi_{+}, \chi_{-}\right\}$for $v_{+}^{\varepsilon}$ and $v_{-}^{\varepsilon}$ and with the phase $\chi(|x|)$ for the Cauchy data $v_{0, \pm}^{\varepsilon}$. The families $w_{ \pm}^{\varepsilon}:=v_{ \pm}^{\varepsilon}-\underline{v}_{ \pm}$introduced at (3.2.2) are also pure families. Let $\mu_{ \pm}\left(y, \theta_{+}, \theta_{-}, d \lambda\right)$ denotes the two-scale Young measures associated to $w_{ \pm}^{\varepsilon}$. The Cauchy data (3.2.6), $w_{0, \pm}^{\varepsilon}:=v_{0, \pm}^{\varepsilon}-\underline{v}_{0, \pm}$, are also pure families and $\mu_{0, \pm}(x, \theta, d \lambda)$ denote the associated measures.

Using equation (3.2.4) and Proposition 4.3.10, we see that

$$
\chi^{\prime}(r+t) \frac{\partial}{\partial \theta_{-}} \mu_{+}=\chi^{\prime}(r-t) \frac{\partial}{\partial \theta_{+}} \mu_{-}=0 .
$$

This implies that $\mu_{+}$[resp. $\mu_{-}$] does not depend on the variable $\theta_{-}$[resp. $\left.\theta_{+}\right]$. Write $\mu_{+}=\mu_{+}\left(y, \theta_{+}, d \lambda\right)$ and $\mu_{-}=\mu_{-}\left(y, \theta_{-}, d \lambda\right)$. They are measures 
on $\mathscr{O} \times \mathbb{T} \times \mathbb{R}$, defined by

$$
\begin{aligned}
& \int \mathscr{A}\left(y, \chi_{+}(y) / \varepsilon\right) f\left(w_{+}^{\varepsilon}(y)\right) d y \rightarrow \iint \mathscr{A}(y, \theta) f(\lambda) \mu_{+}(y, \theta, d \lambda) d y d \theta, \\
& \int \mathscr{A}\left(y, \chi_{-}(y) / \varepsilon\right) f\left(w_{-}^{\varepsilon}(y)\right) d y \rightarrow \iint \mathscr{A}(y, \theta) f(\lambda) \mu_{-}(y, \theta, d \lambda) d y d \theta,
\end{aligned}
$$

for smooth $f$ and $\mathscr{A}$ with compact support.

The following result extends Theorem 3.2.2.

Theorem 4.4.1. The family $w^{\varepsilon}:=\left(w_{+}^{\varepsilon}, w_{-}^{\varepsilon}\right)$ defined in (3.2.2) is pure, and the associated two-scale Young measure $\mu$ on $\Omega \times \mathbb{T}^{2} \times \mathbb{R}^{2}$ satisfies for almost all $\left(y, \theta_{1}, \theta_{2}\right) \in \Omega \times \mathbb{T}^{2}$

$$
\mu\left(y, \theta_{1}, \theta_{2}, d \lambda_{1}, d \lambda_{2}\right)=\mu_{+}\left(y, \theta_{1}, d \lambda_{1}\right) \otimes \mu_{-}\left(y, \theta_{2}, d \lambda_{2}\right) .
$$

Proof. Suppose that $f_{ \pm} \in C_{o}^{\infty}(\mathbb{R})$ and $\mathscr{A}_{ \pm} \in C_{o}^{\infty}\left(\Omega^{*} \times \mathbb{T}\right)$. Introduce

$$
z_{ \pm}^{\varepsilon}(y):=f_{ \pm}\left(w_{ \pm}^{\varepsilon}(y)\right) \mathscr{A}_{ \pm}\left(y, \chi_{ \pm}(y) / \varepsilon\right) .
$$

Then $z_{ \pm}^{\varepsilon}$ converges weakly on $\Omega$ to

$$
\underline{z}_{ \pm}(y)=\int f_{ \pm}(\lambda) \mathscr{A}_{ \pm}(y, \theta) \mu_{ \pm}(y, \theta, d \lambda) d \theta .
$$

Estimates (3.2.4) and the equations $\left(\partial_{t} \pm \partial_{r}\right) \chi_{ \pm}=0$ imply that the families $\left(\partial_{t} \pm \partial_{r}\right) z_{ \pm}^{\varepsilon}$ are bounded in $L^{p^{\prime}}\left(\Omega^{*}\right)$. Similarly, the functions $\Gamma_{k, l} z_{ \pm}^{\varepsilon}$ are bounded in $L^{2}\left(\Omega_{T}\right)$. Thus, estimates (3.2.10) are satisfied, and therefore $z_{+}^{\varepsilon} z_{-}^{\varepsilon}$ converges weakly to $\underline{z}_{+} \underline{z}_{-}$on $\Omega^{*}$. By definition, the $z_{ \pm}^{\varepsilon}$ are compactly supported in $\Omega^{*}$ and therefore

$$
\int f_{+}\left(w_{+}^{\varepsilon}(y)\right) f_{-}\left(w_{-}^{\varepsilon}(y)\right) \mathscr{A}_{+}\left(y, \chi_{+}(y) / \varepsilon\right) \mathscr{A}_{-}\left(y, \chi_{-}(y) / \varepsilon\right) d y \rightarrow \int \underline{z}_{+}(y) \underline{z}_{-}(y) d y \text {. }
$$

With (4.4.7), this yields

$$
\begin{aligned}
& \int f\left(w^{\varepsilon}(y)\right) \mathscr{A}(y, \chi(y) / \varepsilon) d y \\
& \quad \rightarrow \iint f\left(\lambda_{1}, \lambda_{2}\right) \mathscr{A}\left(y, \theta_{1}, \theta_{2}\right) \mu_{+}\left(y, \theta_{1}, d \lambda_{1}\right) \mu_{-}\left(y, \theta_{2}, d \lambda_{2}\right) d y d \theta_{1} d \theta_{2},
\end{aligned}
$$

for any $f \in C_{o}^{\infty}\left(\mathbb{R}^{2}\right)$ of the form $f\left(\lambda_{1}, \lambda_{2}\right)=f_{1}\left(\lambda_{1}\right) f_{2}\left(\lambda_{2}\right)$ and any $\mathscr{A} \in$ $C_{o}^{\infty}\left(\Omega^{*} \times \mathbb{T}^{2}\right)$ of the form $\mathscr{A}\left(y, \theta_{1}, \theta_{2}\right)=\mathscr{A}_{+}\left(y, \theta_{1}\right) \mathscr{A}\left(y, \theta_{2}\right)$. By density of finite linear combinations of such $f$ and $\mathscr{A},(4.4 .8)$ extends to all $f \in C_{o}^{0}\left(\mathbb{R}^{2}\right)$ and $\mathscr{A} \in C_{o}^{0}\left(\Omega^{*} \times \mathbb{T}^{2}\right)$.

This proves that the family $w^{\varepsilon}=\left(w_{+}^{\varepsilon}, w_{-}^{\varepsilon}\right)$ is pure and that the associated measure is given by the right-hand side of (4.4.8). This is equivalent to (4.4.5).

We now summarize the integrability properties which follow from the uniform a priori estimates.

Proposition 4.4.2. One has the following integrability properties:

(i) $\lambda \in L^{2}\left(\Omega_{T} \times \mathbb{T} \times \mathbb{R}, \mu_{ \pm}\right)$for all $T>0$, and $\lambda \in L^{p}\left(\Omega \times \mathbb{T} \times \mathbb{R}, \mu_{ \pm}\right)$.

(ii) $\lambda_{1}^{2}+\lambda_{2}^{2} \in L^{1}\left(\Omega_{T} \times \mathbb{T}^{2} \times \mathbb{R}^{2}, \mu\right)$ for all $T>0$, and $\lambda_{1}+\lambda_{2} \in L^{p}\left(\Omega \times \mathbb{T}^{2} \times\right.$ $\left.\mathbb{R}^{2}, \mu\right)$. 
(iii) $\lambda \in L^{2}\left(\mathbb{R}^{d} \times \mathbb{T} \times \mathbb{R}, \mu_{0, \pm}\right)$.

Proof. One can prove these directly as in Proposition 3.2.5. Alternatively, reason as follows. For $0 \leq \varphi \in C_{o}^{\infty}(\bar{\Omega})$ and $0 \leq f \in C_{o}^{\infty}(\mathbb{R})$, Remark 4.3.3 shows that

$$
\iint \varphi(y) f(\lambda) \mu(y, \theta, d \lambda) d y d \theta=\iint \varphi(y) f(\lambda) \tilde{\mu}(y, d \lambda) d y
$$

where $\tilde{\mu}$ denotes the classical Young measure studied in section 3. Hence, Proposition 4.4.2 follows from Fatou's lemma and the integrability properties with respect to $\tilde{\mu}_{ \pm}$in Lemma 3.2.1 and Proposition 3.2.5.

In a similar way, Proposition 3.2.6 can be extended to the two-scale analysis. Recall the notation $(3.2 .18), \widetilde{F}(y, \lambda):=F\left(\partial_{t} \underline{u}(y)+\lambda\right)$, where $F$ is the nonlinearity of equation (2.1.1).

Proposition 4.4.3. $\widetilde{F}\left(y, \lambda_{1}+\lambda_{2}\right) \in L^{p^{\prime}}\left(\Omega \times \mathbb{T}^{2} \times \mathbb{R}^{2}, \mu\right)$ where $p^{\prime}:=p /(p-1)$ is the conjugate exponent of $p$. Moreover, for $\mathscr{A} \in C_{o}^{\infty}(\bar{\Omega} \times \mathbb{T})$ and $f \in C_{o}^{\infty}(\mathbb{R})$, one has

$$
\begin{aligned}
& \int \mathscr{A}\left(y, \chi_{+}(y) / \varepsilon\right) f\left(w_{+}^{\varepsilon}(y)\right) \tilde{F}\left(y, w_{+}^{\varepsilon}(y)+w_{-}^{\varepsilon}(y)\right) d y \\
& \quad \rightarrow \iint \mathscr{A}\left(y, \theta_{1}\right) f\left(\lambda_{1}\right) \tilde{F}\left(y, \lambda_{1}+\lambda_{2}\right) \mu\left(y, \theta_{1}, \theta_{2}, d \lambda_{1}, d \lambda_{2}\right) d y d \theta_{2} .
\end{aligned}
$$

Proof. For bounded $\widetilde{F},(4.4 .10)$ is a consequence of Proposition 4.3.5. The result for $|\widetilde{F}(y, \lambda)|=O\left(\left|\partial_{t} \underline{u}(y)\right|^{p-1}+|\lambda|^{p-1}\right)$ follows along the usual lines: approximate $\widetilde{F}$ by $\widetilde{F}_{k}:=\zeta\left\{\left(\lambda_{1}+\lambda_{2}\right) / k\right\} \widetilde{F}$ and use the uniform $L^{p}$ bounds for $\partial_{t} \underline{u} \in L^{p}(\Omega)$ and $w_{+}^{\varepsilon}+w_{-}^{\varepsilon}$, together with the integrability properties of Proposition 4.4.2.

4.5. Transport equations. Parallel to Theorem 3.3.1, one has transport equations for the two-scale measures.

Theorem 4.5.1. On $\Omega^{*} \times \mathbb{T} \times \mathbb{R}$, the measures $\mu_{ \pm}$satisfy

$$
\begin{gathered}
2\left(\partial_{t} \pm \partial_{r}\right) \mu_{ \pm} \mp(d-1) r^{-1} \partial_{\lambda}\left\{\lambda \mu_{ \pm}\right\}-\partial_{\lambda}\left\{F_{ \pm}(y, \lambda) \mu_{ \pm}\right\}=0 \\
\mu_{ \pm \mid t=0}=\mu_{0, \pm}
\end{gathered}
$$

where

$$
F_{ \pm}(y, \lambda):=\int \tilde{F}(y, \lambda+\tau) \mu_{\mp}(y, \theta, d \tau) d \theta-\underline{F}(y) .
$$

Remark 4.5.2. In formula (4.5.3), integration in $\theta$ leads to the usual Young measure, as explained in formula (4.3.2). Thus, Definition (4.5.3) of $F_{ \pm}$is equivalent to Definition (3.3.3) and Remark 3.3.2 shows that $F_{ \pm} \in$ $L^{p^{\prime}}\left(\Omega \times \mathbb{T} \times \mathbb{R}, \mu_{ \pm}\right), p^{\prime}=p /(p-1)$. In particular, $F_{ \pm} \mu_{ \pm}$is well defined as a distribution and equation (4.5.1) makes sense in $\mathscr{D}^{\prime}\left(\Omega^{*} \times \mathbb{T} \times \mathbb{R}\right)$. Note that the equations (4.5.1) hold away from the axis $\{x=0\}$.

Proof. Suppose that $f \in C_{o}^{\infty}(\mathbb{R})$ and $\mathscr{A} \in C_{o}^{\infty}(\bar{\Omega} \times \mathbb{T})$ vanishes in a neighborhood of $\{x=0\}$. Multiply equation (3.2.3) by $\mathscr{A}\left(y, \chi_{+}(y) / \varepsilon\right) f^{\prime}\left(w_{ \pm}^{\varepsilon}(y)\right)$, integrate over $\Omega^{*}$ and let $\varepsilon$ tend to 0 . This repeats the successive steps of the 
proof of Theorem 3.3.1. However, we are now using an oscillating test function $\varphi^{\varepsilon}(y):=\mathscr{A}\left(y, \chi_{+}(y) / \varepsilon\right)$. Several remarks must be added.

(1) When integrating by parts, as in steps (a) and (e) of the proof of Theorem 3.3.1, it is of crucial importance to remark that $\left(\partial_{t}+\partial_{r}\right) \chi_{+}=0$ and $\Gamma_{k, l} \chi_{+}=0$, so that $\left(\partial_{t}+\partial_{r}\right) \varphi^{\varepsilon}(y)=\left\{\left(\partial_{t}+\partial_{r}\right) \mathscr{A}\right\}\left(y, \chi_{+}(y) / \varepsilon\right)$ and $\Gamma_{k, l} \varphi^{\varepsilon}(y)=$ $\left\{\Gamma_{k, l} \mathscr{A}\right\}\left(y, \chi_{+}(y) / \varepsilon\right)$ are bounded.

(2) The weak limit of $w_{ \pm}^{\varepsilon}$ is 0 . According to Remark 4.3.3 this implies that

$$
\int_{\mathbb{R} \times \mathbb{T}} \lambda \mu_{ \pm}(y, \theta, d \lambda) d \theta=0, \quad \text { a.e. on } \Omega \text {. }
$$

(3) On the other hand, Proposition 4.4.3 is used to prove the analogue of (3.3.9).

Summarizing, we obtain

$$
\begin{aligned}
& -2 \iint\left\{\left(\partial_{t}+\partial_{r}\right) \mathscr{A}(y, \theta)\right\} f(\lambda) \mu_{+}(y, \theta, d \lambda) d \theta d y \\
& \quad+(d-1) \iint r^{-1} \mathscr{A}(y, \theta) \lambda f^{\prime}(\lambda) \mu_{+}(y, \theta, d \lambda) d \theta d y \\
& \quad+\iint \mathscr{A}(y, \theta) f^{\prime}(\lambda) F_{+}(y, \lambda) \mu_{+}(y, \theta, d \lambda) d \theta d y \\
& -2 \iint \mathscr{A}(0, x, \theta) f(\lambda) \mu_{0,+}(x, \theta, d \lambda) d \theta d x=0 .
\end{aligned}
$$

This is the weak form of (4.5.1), (4.5.2) and Theorem 4.5.1 is proved.

The next step is to prove a propagation inequality for the variance of $\mu_{ \pm}$. As a preliminary, we need an equation for the weak profiles $\mathscr{W}_{ \pm}$of $w_{ \pm}^{\varepsilon}$. According to Remark 4.3.6, they are given by

$$
\mathscr{W}_{ \pm}(y, \theta):=\int_{\mathbb{R}} \lambda \mu_{ \pm}(y, \theta, d \lambda), \quad \text { a.e. on } \Omega \times \mathbb{T} \text {. }
$$

Similarly, the weak profiles for the initial data are

$$
\mathscr{W}_{0, \pm}(x, \theta):=\int_{\mathbb{R}} \lambda \mu_{0, \pm}(x, \theta, d \lambda), \quad \text { a.e. on } \Omega \times \mathbb{T} \text {. }
$$

Proposition 4.5.3. The weak profiles $\mathscr{W}_{ \pm}$belong to $L^{2}\left(\Omega_{T} \times \mathbb{T}\right)$ for all $T>0$, and to $L^{p}(\Omega \times \mathbb{T})$. With $\Gamma_{k, l}$ defined in (2.1.3), $\Gamma_{k, l}\left(x, \partial_{x}\right) \mathscr{W}_{ \pm}(t, x, \theta) \in$ $L^{2}\left(\Omega_{T} \times \mathbb{T}\right)$. Moreover on $\Omega^{*}$ they satisfy

$$
\begin{gathered}
2\left(\partial_{t} \pm \partial_{r}\right) \mathscr{W}_{ \pm} \pm(d-1) r^{-1} \mathscr{W}_{ \pm}+\mathscr{F}_{ \pm}=0, \\
\mathscr{W}_{ \pm \mid t=0}=\mathscr{W}_{0, \pm},
\end{gathered}
$$

where $\mathscr{F}_{ \pm} \in L^{p^{\prime}}(\Omega \times \mathbb{T}), p^{\prime}:=p /(p-1)$, is given by

$$
\mathscr{F}_{ \pm}(y, \theta):=\int_{\mathbb{R}} F_{ \pm}(y, \lambda) \mu_{ \pm}(y, \theta, d \lambda) \text {, a.e. on } \Omega \times \mathbb{T} \text {. }
$$

Remark 4.5.4. The functions $\mathscr{F}_{ \pm}$are determined by $\mu_{ \pm}$and not by $\mathscr{W}_{ \pm}$so the equations (4.5.8), (4.5.10) do not form a closed system for the $\mathscr{W}_{ \pm}$. A closed system for $\left(\mathscr{W}_{ \pm}, \underline{u}\right)$ is given in Proposition 4.6.2. 
Proof. The integrability properties of $\mathscr{W}_{ \pm}$follow from Proposition 4.4.2. Remark 4.5.2 shows that $F_{ \pm} \in L^{p^{\prime}}\left(\Omega \times \mathbb{T} \times \mathbb{R}, \mu_{ \pm}\right)$which implies that $\mathscr{F}_{ \pm} \in$ $L^{p^{\prime}}(\Omega \times \mathbb{T})$.

Using Proposition 4.4.2, one can extend (4.5.5) to $f(\lambda) \equiv \lambda$ which shows that for all $\mathscr{A} \in C_{0}^{\infty}(\bar{\Omega} \times \mathbb{T})$ (4.5.11)

$$
\begin{aligned}
-2 \int & \left\{\left(\partial_{t}+\partial_{r}\right) \mathscr{A}(y, \theta)\right\} \mathscr{W}_{+}(y, \theta) d \theta d y \\
& +(d-1) \int r^{-1} \mathscr{A}(y, \theta) \mathscr{W}_{+}(y, \theta) d \theta d y \\
& +\int \mathscr{A}(y, \theta) \mathscr{F}_{+}(y, \theta) d \theta d y-2 \int \mathscr{A}(0, x, \theta) \mathscr{W}_{0,+}(x, \theta) d \theta d x=0 .
\end{aligned}
$$

This is the weak form of (4.5.8), (4.5.9).

Moreover, the definition (4.1.5) of weak profiles and the property that $\Gamma_{k, l} \chi_{ \pm}$ $=0$ show that the weak profile of the family $\Gamma_{k, l} w_{ \pm}^{\varepsilon}$ is $\Gamma_{k, l}\left(x, \partial_{x}\right) \mathscr{W}_{ \pm}(t, x, \theta)$. The estimates (2.1.5) imply that $\Gamma_{k, l} v_{ \pm}^{\varepsilon}$ are bounded in $L^{2}\left(\Omega_{T}\right)$. Thus $\Gamma_{k, l} w_{ \pm}^{\varepsilon}$ is bounded in $L^{2}\left(\Omega_{T}\right)$. By Proposition 4.1.4 this implies that $\Gamma_{k, l} \mathscr{W}_{ \pm}$belongs to $L^{2}\left(\Omega_{T} \times \mathbb{T}\right)$. The proof of Proposition 4.5.3 is complete.

Theorem 4.5.5. Let $\sigma_{ \pm}$denote the variance of $\mu_{ \pm}$,

$$
\sigma_{ \pm}(y, \theta):=\int_{\mathbb{R}}\left\{\lambda-\mathscr{W}_{ \pm}(y, \theta)\right\}^{2} \mu_{ \pm}(y, \theta, d \lambda) \text {, a.e. on } \Omega \times \mathbb{T} \text {. }
$$

Then $\sigma_{ \pm} \in L^{1}\left(\Omega_{T} \times \mathbb{T}\right) \cap L^{p / 2}(\Omega \times \mathbb{T}),\left(\partial_{t} \pm \partial_{r}\right) \sigma_{ \pm} \in r^{-1} L^{p / 2}(\Omega \times \mathbb{T})+L^{1}(\Omega \times \mathbb{T})$ and there is a constant $c>0$ such that

$$
\left(\partial_{t} \pm \partial_{r}\right) \sigma_{ \pm} \pm(d-1) r^{-1} \sigma_{ \pm}+c\left(\sigma_{ \pm}\right)^{p / 2} \leq 0, \quad \text { a.e. on } \Omega^{*} \times \mathbb{T}
$$

Moreover, the trace of $\sigma_{ \pm}$on $t=0$ is the variance $\sigma_{0, \pm}$ of $\mu_{0, \pm}$.

Proof. The integrability properties of $\sigma_{+}$follow from Lemma 4.4.2. Because the expectation of $\mu_{+}$vanishes, $\sigma_{+}=\mathscr{Z}_{+}-\mathscr{W}_{+}^{2}$ where

$$
\mathscr{Z}_{+}(y, \theta):=\int_{\mathbb{R}} \lambda^{2} \mu_{+}(y, \theta, d \lambda), \quad \text { a.e. on } \Omega \times \mathbb{T} \text {. }
$$

The estimates of Lemma 4.4.2 allow us to extend (4.5.5) to $f(\lambda)=\lambda^{2} / 2$. This yields

$$
\left(\partial_{t}+\partial_{r}\right) \mathscr{Z}_{+}+(d-1) r^{-1} \mathscr{Z}_{+}+\mathscr{G}_{+}=0, \quad \mathscr{Z}_{+\mid t=0}=\mathscr{Z}_{0,+},
$$

with

$$
\mathscr{G}_{+}(y, \theta):=\int_{\mathbb{R}} \lambda F_{+}(y, \lambda) \mu_{+}(y, \theta, d \lambda) \in L^{1}(\Omega \times \mathbb{T}) .
$$

On the other hand, equation (4.5.8) shows that $\mathscr{W}_{+}^{2}$ satisfies

$$
\left(\partial_{t}+\partial_{r}\right) \mathscr{W}_{+}^{2}+(d-1) r^{-1} \mathscr{W}_{+}^{2}+\mathscr{W}_{+} \mathscr{F}_{+}=0, \quad \mathscr{W}_{+\mid t=0}^{2}=\mathscr{W}_{0,+}^{2} .
$$

Substracting from (4.5.15) yields

$$
\left(\partial_{t}+\partial_{r}\right) \sigma_{+}+(d-1) r^{-1} \sigma_{+}+\mathscr{H}_{+}=0, \quad \sigma_{+\mid t=0}=\sigma_{0,+},
$$

where $\mathscr{H}_{+}:=\mathscr{G}_{+}-\mathscr{W}_{+} \mathscr{F}_{+} \in L^{1}(\Omega \times \mathbb{T})$. Thus $\left(\partial_{t} \pm \partial_{r}\right) \sigma_{ \pm} \in r^{-1} L^{p / 2}(\Omega \times \mathbb{T})+$ $L^{1}(\Omega \times \mathbb{T})$. 
Using (4.5.3) yields

$$
\mathscr{F}_{+}(y, \theta)=\int \widetilde{F}(y, \lambda+\tau) \mu_{+}(y, \theta, d \lambda) \mu_{-}(y, \eta, d \tau) d \eta-\underline{F}(y),
$$

$$
\mathscr{G}_{+}(y, \theta)=\int \lambda \widetilde{F}(y, \lambda+\tau) \mu_{+}(y, \theta, d \lambda) \mu_{-}(y, \eta, d \tau) d \eta-\underline{F}(y) \mathscr{W}_{+}(y, \theta) .
$$

Thus $\mathscr{H}_{+}=\mathscr{G}_{+}-\mathscr{W}_{+} \mathscr{F}_{+}$is given by

$$
\mathscr{H}_{+}(y, \theta)=\int\left\{\lambda-\mathscr{W}_{+}(y, \theta)\right\} \widetilde{F}(y, \lambda+\tau) \mu_{+}(y, \theta, d \lambda) \mu_{-}(y, \eta, d \tau) d \eta .
$$

Because the expectation of $\left(\lambda-\mathscr{W}_{+}\right)$is 0 , one has

$$
\mathscr{H}_{+}(y, \theta)=\int H(y, \theta, \lambda, \tau) \mu_{+}(y, \theta, d \lambda) \mu_{-}(y, \eta, d \tau) d \eta
$$

with

(4.5.23) $H(y, \theta, \lambda, \tau)=\left\{\lambda-\mathscr{W}_{+}(y, \theta)\right\}\left\{\widetilde{F}(y, \lambda+\tau)-\widetilde{F}\left(y, W_{+}(y, \theta)+\tau\right)\right\}$.

Estimate (3.3.24) implies that

$$
H(y, \theta, \lambda, \tau) \geq c\left\{\lambda-\mathscr{W}_{+}(y, \theta)\right\}^{p} .
$$

Using Jensen's inequality, we obtain that

$$
\mathscr{H}_{+}(y, \theta) \geq c \int\left\{\lambda-\mathscr{W}_{+}(y, \theta)\right\}^{p} \mu_{+}(y, \theta, d \lambda) \mu_{-}(y, \eta, d \tau) d \eta \geq c \sigma_{+}^{p / 2}(y, \theta) .
$$

Plugging this estimate into (4.5.18) gives (4.5.13) and Theorem 4.5.5 is proved.

Corollary 4.5.6. Suppose in addition that Assumption 2.4.1 is satisfied and that $\beta:=(d-1)(p-2) / 2 \geq 1$. Then $\sigma_{ \pm} \equiv 0$. Moreover, $\mathscr{W}_{+} \equiv 0$ in the cone $\mathscr{C}:=\{t>|x|\}$.

Proof. (a) Since the Cauchy data are of the form (2.4.1), (2.4.2), and satisfy Assumption 2.4.1, the Cauchy data (2.2.13) $v_{0, \pm}^{\varepsilon}$ satisfy

$$
v_{0, \pm}^{\varepsilon}(x):=\frac{1}{2}\left(u_{1}^{\varepsilon} \mp \partial_{r} u_{0}^{\varepsilon}\right)(x)=\mathscr{V}_{0, \pm}(x, \chi(|x|) / \varepsilon)+r_{0, \pm}^{\varepsilon}(x)
$$

$$
\begin{gathered}
\mathscr{V}_{0, \pm}(x, \theta):=\frac{1}{2}\left\{\mathscr{U}_{1}(x, \theta) \mp \chi^{\prime}(|x|) \partial_{\theta} \mathscr{U}_{0}(x, \theta)\right\}, \\
r_{0, \pm}^{\varepsilon} \rightarrow 0 \text { strongly in } L^{2}\left(\mathbb{R}^{d}\right) \text { as } \varepsilon \rightarrow 0 .
\end{gathered}
$$

This shows that the Cauchy data $w_{0, \pm}^{\varepsilon}:=v_{0, \pm}^{\varepsilon}-\underline{v}_{0, \pm}$ satisfy

$$
\begin{gathered}
w_{0, \pm}^{\varepsilon}(x):=\mathscr{W}_{0, \pm}(x, \chi(|x|) / \varepsilon)+r_{0, \pm}^{\varepsilon}(x), \\
\mathscr{W}_{0, \pm}(x, \theta):=\mathscr{V}_{0, \pm}(x, \theta)-\underline{v}_{0, \pm}(x) .
\end{gathered}
$$

The weak limit $\underline{v}_{0, \pm}$ is the mean of $\mathscr{V}_{0, \pm}$ :

$$
\underline{v}_{0, \pm}(x)=\int \mathscr{V}_{0, \pm}(x, \theta) d \theta
$$


In particular, the families $\left\{w_{0, \pm}^{\varepsilon}\right\}$ are pure with respect to the phase $\chi(|x|)$ and the associated two-scale Young measures $\mu_{0, \pm}$ are the Dirac mass at $\lambda=$ $\mathscr{W}_{0, \pm}(x, \theta)$. In particular the variances $\sigma_{0, \pm}$ vanish.

(b) The variances $\sigma_{ \pm}$satisfy the tranport inequalities (4.5.13). Their Cauchy data $\sigma_{0, \pm}$ vanish, and therefore $\sigma_{-}=0$ a.e. on $\Omega^{*} \times \mathbb{T}$, and $\sigma_{+}=0$ a.e. on $(\Omega \cap\{t<|x|\}) \times \mathbb{T}$.

(c) Inside the light cone $\{t>|x|\}$, the proof of Proposition 3.4.2 can be repeated to conclude that $\sigma_{+}=0$ there too. Alternatively, Theorem 2.3.3 tells us that $w_{+}^{\varepsilon}$ converges strongly to 0 , in $L_{\text {loc }}^{2}\left(\Omega^{*}\right)$. This implies that the Young measure $\mu_{+}(y, d \lambda)$ is the Dirac mass at $\lambda=0$ for almost all $y \in \Omega$, and therefore $\mu_{+}(y, \theta, d \lambda)$ is also the Dirac mass at $\lambda=0$ for almost all $(y, \theta) \in \Omega \times \mathbb{T}$. This proves that $\sigma_{+}$and $\mathscr{W}_{+}$vanish almost everywhere on $\{t>|x|\} \times \mathbb{T}$.

With part (b), this shows that $\sigma_{+}$vanishes almost everywhere on $\Omega \times \mathbb{T}$.

4.6. Profile equations. In addition to the assumptions listed at the beginning of $\S 4.4$, we now assume that the superlinear condition $\beta:=(d-1)(p-2) / 2 \geq$ 1 is fulfilled and that the Cauchy data are of the form (2.4.1), (2.4.2), and satisfy Assumption 2.4.1. In this subsection, we derive the profile equations (2.4.7), prove uniqueness of this system, and prove extra regularity in $\theta$ under the corresponding assumption for the data. This will complete the proof of Theorem 2.4.2.

Proposition 4.6.1. (i) $\underline{u}$ satisfies

$$
\underline{u} \in H^{1}\left(\Omega_{T}\right) \text { for all } T>0, \quad \partial_{t} \underline{u} \in L^{p}(\Omega),
$$

and

$$
\square \underline{u}+\underline{F}=0 \text { on } \Omega, \quad \underline{u}_{\mid t=0}=\underline{u}_{0}, \quad \partial_{t} \underline{u}_{\mid t=0}=\underline{u}_{1} .
$$

(ii) The profiles $\mathscr{W}_{ \pm}$satisfy

$$
\begin{gathered}
\mathscr{W}_{ \pm} \in L^{2}\left(\Omega_{T} \times \mathbb{T}\right) \text { for all } T>0 \text { and } \mathscr{W}_{ \pm} \in L^{p}(\Omega \times \mathbb{T}) \\
\mathscr{W}_{+}=0 \text { in } \mathscr{C}=\{t>|x|\}, \\
\int \mathscr{W}_{ \pm}(y, \theta) d \theta=0 \text { for almost all } y \in \Omega,
\end{gathered}
$$

and

$$
X_{ \pm} \mathscr{W}_{ \pm}+\mathscr{F}_{ \pm}=0 \text { on } \Omega^{*}, \quad \mathscr{W}_{ \pm \mid t=0}=\mathscr{W}_{0, \pm}
$$

where

$$
\begin{gathered}
\underline{F}(y):=\iint F\left(\partial_{t} \underline{u}(y)+\mathscr{W}_{+}(y, \theta)+\mathscr{W}_{-}(y, \tau)\right) d \theta d \tau \\
\mathscr{F}_{ \pm}(y, \theta):=\int F\left(\partial_{t} \underline{u}(y)+\mathscr{W}_{ \pm}(y, \theta)+\mathscr{W}_{\mp}(y, \tau)\right) d \tau-\underline{F}(y), \\
X_{ \pm}:=2\left(\partial_{t} \pm \partial_{r}\right) \pm(d-1) r^{-1}
\end{gathered}
$$

Remark 4.6.2. The transport equations (4.6.6) hold on the open set $\Omega^{*}$. They are singular at $x=0$. 
Proof. The integrability properties of $\mathscr{W}_{ \pm}$are proved in Proposition 4.5.3. (4.6.4) was proved in Corollary 4.5.6. The integral in (4.6.5) is the weak limit of $w_{ \pm}^{\varepsilon}$, and therefore vanishes almost everywhere.

Corollary 4.5.6 shows that $\sigma_{ \pm}=0$ and therefore $\mu_{ \pm}(y, \theta)$ is the Dirac mass at $\lambda=\mathscr{W}_{ \pm}(y, \theta)$. Therefore, the definition (4.5.10) of the functions $F_{ \pm}$ simplifies to

$$
\begin{aligned}
\mathscr{F}_{ \pm}(y, \theta) & =F_{ \pm}\left(y, \mathscr{W}_{ \pm}(y, \theta)\right) \\
& :=\int \widetilde{F}\left(y, \mathscr{W}_{ \pm}(y, \theta)+\mathscr{W}_{\mp}(y, \tau)\right) d \tau-\underline{F}(y) .
\end{aligned}
$$

Similarly, $\underline{F}$, the weak limit of $F\left(\partial_{t} u^{\varepsilon}\right)=F\left(\partial_{t} \underline{u}^{\varepsilon}+w_{+}^{\varepsilon}+w_{-}^{\varepsilon}\right)$, is given by (4.6.7). Hence equations (4.6.6) follow from Proposition 4.5.3. That $\underline{u}$ satisfies (4.6.1), (4.6.2) is demonstrated in (2.2.6), (2.2.7).

Proposition 4.6.3. (i) (Uniqueness for the profile equations). The system of equations (4.6.2), (4.6.6) uniquely determines ( $\left.\underline{u}, \mathscr{W}_{ \pm}\right)$such that (4.6.1), (4.6.3), (4.6.4), (4.6.5) are satisfied.

(ii) In addition, such solutions satisfy

$$
\begin{gathered}
\nabla \underline{u} \in L^{\infty}\left(\left[0,+\infty\left[; L^{2}\left(\mathbb{R}^{d}\right)\right),\right.\right. \\
\mathscr{W}_{ \pm} \in L^{\infty}\left(\left[0,+\infty\left[; L^{2}\left(\mathbb{R}^{d} \times \mathbb{T}\right)\right) .\right.\right.
\end{gathered}
$$

Remark 4.6.4. Estimates (4.6.10), (4.6.11) could be deduced from energy estimates for $u^{\varepsilon}$. However, we include a direct proof from equations (4.6.2), (4.6.6), because it provides the energy estimates which would be useful for a direct proof of existence of solutions.

Proof. (a) Formally, one has

$$
\begin{aligned}
\int_{\Omega_{\underline{t}}} & \square z \cdot \partial_{t} z d t d x \\
\quad= & \frac{1}{2} \int_{\mathbb{R}^{d}}\left|\nabla_{t, x} z(\underline{t}, x)\right|^{2} d x-\frac{1}{2} \int_{\mathbb{R}^{d}}\left|\nabla_{t, x} z(0, x)\right|^{2} d x .
\end{aligned}
$$

Using regularizations (4.6.12) is easily justified for those functions $z$ such that $z \in H^{1}\left(\Omega_{T}\right)$ for all $T>0, \square z \in L^{p^{\prime}}(\Omega)$ and $\partial_{t} z \in L^{p}(\Omega)$. This implies that such $z$ satisfy $\nabla z \in L^{\infty}\left(\left[0,+\infty\left[; L^{2}\left(\mathbb{R}^{d}\right)\right)\right.\right.$. In particular, $\underline{u}$ satisfies (4.6.10).

(b) Formal integration by parts in the region $\{|x|>\rho\}, \rho>0$, where $X_{-}$ is regular yields

$$
\begin{aligned}
& \int_{\{|x|>\rho\} \cap \Omega_{\underline{t}} \times \mathbb{T}} X_{-} \mathscr{Z}_{-} \cdot \mathscr{Z}_{-} d t d x d \theta \\
&=\int_{\{|x|>\rho\} \times \mathbb{T}}\left|\mathscr{Z}_{-}(\underline{t}, x, \theta)\right|^{2} d x d \theta \\
&-\left.\int_{\{|x|>\rho\} \times \mathbb{T}} \mathscr{Z}_{-}(0, x, \theta)\right|^{2} d x d \theta \\
&+\int_{0}^{\underline{t}} \int_{\{|x|=\rho\} \times \mathbb{T}}\left|\mathscr{Z}_{-}(t, x, \theta)\right|^{2} d t d x d \theta .
\end{aligned}
$$


This formula extends to $\mathscr{Z}_{-}$such that $\mathscr{Z}_{-} \in L^{p}(\Omega \times \mathbb{T})$, and $X_{-} \mathscr{Z}_{-} \in L^{p^{\prime}}(\Omega \times$ $\mathbb{T})$. Since the last integral is nonnegative, passing to the limit as $\rho \rightarrow 0$ yields

$$
\begin{aligned}
& \int_{\Omega_{\underline{t}} \times \mathbb{T}} X_{-} \mathscr{Z}_{-} \cdot \mathscr{Z}_{-} d t d x d \theta \\
& \quad \geq \int_{\mathbb{R}^{d} \times \mathbb{T}}\left|\mathscr{Z}_{-}(\underline{t}, x, \theta)\right|^{2} d x d \theta-\left.\int_{\mathbb{R}^{d} \times \mathbb{T}} \mathscr{Z}_{-}(0, x, \theta)\right|^{2} d x d \theta .
\end{aligned}
$$

This implies that such $\mathscr{Z}_{-}$belong to $L^{\infty}\left(\left[0,+\infty\left[; L^{2}\left(\mathbb{R}^{d}\right)\right)\right.\right.$. In particular, $\mathscr{W}_{-}$ satisfies (4.6.11).

(c) For the plus sign, integrate by parts in $\{|x|>t+\rho\}$ to find

$$
\begin{aligned}
\int_{\{|x|>\rho+t\} \cap \Omega_{\underline{t}} \times \mathbb{T}} & X_{+} \mathscr{Z}_{+} \cdot \mathscr{Z}_{+} d t d x d \theta \\
= & \int_{\{|x|>\rho+\underline{t}\} \times \mathbb{T}}\left|\mathscr{Z}_{+}(\underline{t}, x, \theta)\right|^{2} d x d \theta \\
& -\left.\int_{\{|x|>\rho\} \times \mathbb{T}} \mathscr{Z}_{+}(0, x, \theta)\right|^{2} d x d \theta .
\end{aligned}
$$

This formula is justified for those $\mathscr{Z}_{+}$such that $\mathscr{Z}_{+} \in L^{p}(\Omega \times \mathbb{T})$, and $X_{+} \mathscr{Z}_{+-} \in$ $L^{p^{\prime}}(\Omega \times \mathbb{T})$. Passing to the limit as $\rho \rightarrow 0$ yields

$$
\begin{aligned}
\int_{\{|x|>t\} \cap \Omega_{\underline{t}} \times \mathbb{T}} & X_{+} \mathscr{Z}_{+} \cdot \mathscr{Z}_{+} d t d x d \theta \\
= & \int_{\{|x|>\underline{t}\} \times \mathbb{T}}\left|\mathscr{Z}_{+}(\underline{t}, x, \theta)\right|^{2} d x d \theta \\
& -\left.\int_{\mathbb{R}^{d} \times \mathbb{T}} \mathscr{Z}_{+}(0, x, \theta)\right|^{2} d x d \theta .
\end{aligned}
$$

If in addition $\mathscr{Z}_{+}=0$ in $\{t>|x|\}$, then

$$
\begin{aligned}
& \int_{\Omega_{\underline{t}} \times \mathbb{T}} X_{+} \mathscr{Z}_{+} \cdot \mathscr{Z}_{+} d t d x d \theta \\
& \quad=\int_{\mathbb{R}^{d} \times \mathbb{T}}\left|\mathscr{Z}_{+}(\underline{t}, x, \theta)\right|^{2} d x d \theta-\int_{\mathbb{R}^{d} \times \mathbb{T}}\left|\mathscr{Z}_{+}(0, x, \theta)\right|^{2} d x d \theta .
\end{aligned}
$$

This implies that such $\mathscr{Z}_{+}$'s belong to $L^{\infty}\left(\left[0,+\infty\left[; L^{2}\left(\mathbb{R}^{d}\right)\right)\right.\right.$. In particular, $\mathscr{W}_{+}$satisfies (4.6.11).

(d) Assume that $\left(\underline{u}^{(1)}, \mathscr{W}_{ \pm}^{(1)}\right)$ and $\left(\underline{u}^{(2)}, \mathscr{W}_{ \pm}^{(2)}\right)$ are two solutions of (4.6.2), (4.6.6) which satisfy $(4.6 .1),(4.6 .3),(4.6 .4),(4.6 .5)$. Introduce $z:=\underline{u}^{(2)}-\underline{u}^{(1)}$, $\mathscr{Z}_{ \pm}:=\mathscr{W}_{ \pm}^{(2)}-\mathscr{W}_{ \pm}^{(1)}$. Then

$$
\begin{gathered}
\square z+g=0, \quad z_{\mid t=0}=0, \quad \partial_{t} z_{\mid t=0}=0, \\
X_{ \pm} \mathscr{Z}_{ \pm}+\mathscr{G}_{ \pm}=0, \quad \mathscr{Z}_{ \pm \mid t=0}=0,
\end{gathered}
$$

where $g:=\underline{F}^{(2)}-\underline{F}^{(1)}, \mathscr{G}_{ \pm}:=\mathscr{F}_{ \pm}^{(2)}-\mathscr{F}_{ \pm}^{(1)}$.

Remark that $g \in L^{p^{\prime}}(\Omega), \mathscr{G}_{ \pm} \in L^{p^{\prime}}(\Omega \times \mathbb{T})$ and $\mathscr{Z}_{+}=0$ on $\{t>|x|\}$. 
Using (4.6.12), (4.6.13) and (4.6.16) yields

$$
\begin{aligned}
& \frac{1}{2} \int_{\mathbb{R}^{d}}\left|\nabla_{t, x} z(\underline{t}, x)\right|^{2} d x+\int_{\mathbb{R}^{d} \times \mathbb{T}}\left|\mathscr{Z}_{+}(\underline{t}, x, \theta)\right|^{2} d x d \theta \\
& +\int_{\mathbb{R}^{d} \times \mathbb{T}}\left|\mathscr{Z}_{-}(\underline{t}, x, \theta)\right|^{2} d x d \theta \leq-A
\end{aligned}
$$

where

$$
\begin{aligned}
A:= & \int_{\Omega_{\underline{t}}} g \partial_{t} z d t d x+\int_{\Omega_{\underline{t}} \times \mathbb{T}} X_{+} \mathscr{Z}_{+} \cdot \mathscr{Z}_{+} d t d x d \theta \\
& +\int_{\Omega_{\underline{t}} \times \mathbb{T}} X_{-} \mathscr{Z}_{-} \cdot \mathscr{Z}_{-} d t d x d \theta .
\end{aligned}
$$

Because $\partial_{t} \underline{u}$ and $\mathscr{W}$ are known to be in $L^{p}(\Omega)$, the functions $g \cdot \partial_{t} z$ and $\mathscr{G}_{ \pm} \mathscr{Z}_{ \pm}$are integrable. Using (4.6.7), (4.6.8) and (4.6.5), the sum in (4.6.20) can be written as

$$
\int_{\Omega_{\underline{t}} \times \mathbb{T} \times \mathbb{T}} H(t, x, \theta, \tau) d t d x d \theta d \tau
$$

where the integrable function $H$ is given by

$$
H(y, \theta, \tau):=\left\{F^{(2)}-F^{(1)}\right\}\left\{\partial_{t} z(y)+\mathscr{Z}_{+}(y, \theta)+\mathscr{Z}_{-}(y, \tau)\right\},
$$

where

$$
F^{(i)}(y, \theta, \tau):=F\left(\partial_{t} \underline{u}^{(i)}(y)+\mathscr{W}_{+}^{(i)}(y, \theta)+\mathscr{W}_{-}^{(i)}(y, \tau)\right) .
$$

By convexity of $F, H$ is nonnegative almost everywhere, and thus $A$ is nonnegative too. Thus the left-hand side of (4.6.19) vanishes and uniqueness follows.

The proof of Proposition 4.6.3 is complete.

The integrability estimates for $\mathscr{W}_{ \pm}$can be sharpened. Recall that $\beta:=$ $(d-1)(p-2) / 2$.

\section{Proposition 4.6.5. Let}

$$
\sigma_{ \pm}(y):=\int\left\{\mathscr{W}_{+}(y, \theta)\right\}^{2} d \theta \text { and } \sigma_{0, \pm}(x):=\int\left\{\mathscr{W}_{0,+}(x, \theta)\right\}^{2} d \theta
$$

Then $\sigma_{+}$vanishes for $t>|x|=r$, and for $t<|x|$ one has almost everywhere

$$
\begin{gathered}
\sigma_{+}(t, x) \leq\{(r-t) / r\}^{d-1} \sigma_{0,+}(x-t \hat{x}), \\
\sigma_{-}(t, x) \leq\{(r+t) / r\}^{d-1} \sigma_{0,-}(x+t \hat{x})
\end{gathered}
$$

where $\hat{x}:=x /|x|$. In addition, for all almost $(t, x)$ one has

$$
\begin{gathered}
\sigma_{+}(t, x) \leq c t^{-(d-1) / \beta}, \\
\sigma_{-}(t, x) \leq c\{(r+t) / r t\}^{(d-1) / \beta} .
\end{gathered}
$$

Remark 4.6.6. In this statement, $\sigma_{ \pm}$is the variance of the one-scale Young measure $\mu_{ \pm}(y, d \lambda)$ associated to $w_{ \pm}^{\varepsilon}$.

Proof. Equation (4.6.6) implies that $\sigma_{ \pm}$satisfies

$$
\left(\partial_{t} \pm \partial_{r}\right) \sigma_{ \pm} \pm(d-1) r^{-1} \sigma_{ \pm}+\int \mathscr{W}_{ \pm} F_{ \pm} d \theta=0, \quad \sigma_{ \pm \mid t=0}=\sigma_{0, \pm}
$$


Because the mean in $\theta$ of $W_{ \pm}$vanishes, we see that

$$
\int \mathscr{W}_{ \pm} F_{ \pm} d \theta=\int H_{ \pm}(y, \theta, \tau) d \theta d \tau
$$

where

$$
\begin{aligned}
& H_{ \pm}(y, \theta, \tau) \\
& \quad:=\left\{F\left(\partial_{t} \underline{u}(y)+\mathscr{W}_{+}(y, \theta)+\mathscr{W}_{-}(y, \tau)\right)-F\left(\partial_{t} \underline{u}(y)+\mathscr{W}_{\mp}(y, \tau)\right)\right\} \mathscr{W}_{ \pm}(y, \theta) .
\end{aligned}
$$

Then (3.3.24) implies that $H \geq c\{W(y, \theta)\}^{p}$, so that $\zeta_{+}:=r^{(d-1)} \sigma_{ \pm}$satisfies

$$
\left(\partial_{t} \pm \partial_{r}\right) \zeta_{ \pm}+c r^{-\beta}\left(\zeta_{ \pm}\right)^{p / 2} \leq 0, \text { with } \beta:=(d-1)(p-2) / 2 \geq 1 .
$$

Integrate (4.6.27) along characteristics, using the estimate

$$
\int_{0}^{t}(r-t+s)^{-\beta} d s \geq t r^{-\beta}
$$

In polar coordinates and suppressing $\hat{x}$, we obtain

$$
\zeta_{+}(0, r-t)^{1-p / 2}-\zeta_{+}(t, r)^{1-p / 2}+c \frac{p-2}{2} t r^{-\beta} \leq 0 .
$$

This implies that $\zeta_{+}(t, r) \leq \zeta_{+}(0, r-t)$ and also that

$$
\zeta_{+}(t, r) \leq c^{\prime} r^{2 \beta /(p-2)} t^{-2 /(p-2)} \text {. }
$$

Hence

$$
\sigma_{+}(t, r) \leq(r-t)^{(d-1)} r^{(1-d)} \sigma_{+}(0, r-t) \leq \sigma_{+}(0, r-t)
$$

and

$$
\sigma_{+}(t, r) \leq c^{\prime} r^{(1-d)+2 \beta /(p-2)} t^{(1-p / 2)}=c^{\prime} t^{-2 /(p-2)} .
$$

Similarly, the estimate

$$
\int_{0}^{t}(r+t-s)^{-\beta} d s \geq c_{1} t r^{-(\beta-1)}(r+t)^{-1}
$$

implies that

$$
\zeta_{-}(0, r+t)^{1-p / 2}-\zeta_{-}(t, r)^{1-p / 2}+c^{\prime} \frac{p-2}{2} \operatorname{tr}^{1-\beta}(r+t)^{-1} \leq 0 .
$$

Thus $\zeta_{-}(t, r) \leq \zeta_{-}(0, r+t)$ and $\zeta_{-}(t, r) \leq c^{\prime} r^{d-1}\{(r+1) / r t\}^{(d-1) / \beta}$, proving (4.6.24) and (4.6.26).

We next assume more smoothness on the data.

Assumption 4.6.7. The initial profiles in (4.5.27) satisfy $\mathscr{V}_{0, \pm} \in L^{2}\left(\mathbb{R}^{d} \times \mathbb{T}\right)$ and $\partial_{\theta} \mathscr{V}_{0, \pm} \in L^{2}\left(\mathbb{R}^{d} \times \mathbb{T}\right)$.

Proposition 4.6.8. (i) Under Assumption 4.6.7,

$$
\partial_{\theta} \mathscr{W}_{ \pm} \in L^{\infty}\left(\left[0,+\infty\left[; L^{2}\left(\mathbb{R}^{d} \times \mathbb{T}\right)\right)\right.\right. \text {. }
$$

(ii) If, in addition, $\partial_{\theta} \mathscr{V}_{0, \pm} \in L^{\infty}\left(\mathbb{R}^{d} \times \mathbb{T}\right)$, then $\partial_{\theta} \mathscr{W}_{ \pm}$and $\mathscr{W}_{ \pm}$are bounded on any compact set $\omega \subset \Omega^{*}$.

Proof. Reasoning formally, equation (4.6.6) yields

$$
X_{ \pm} \mathscr{W}_{ \pm}+F_{ \pm}\left(y, \mathscr{W}_{ \pm}\right)-\underline{F}(y)=0,
$$


with

$$
F_{ \pm}(y, \lambda):=\int F\left(\partial_{t} \underline{u}(y)+\mathscr{W}_{\mp}(y, \tau)+\lambda\right) d \tau .
$$

Thus $\mathscr{Z}_{ \pm}:=\partial_{\theta} \mathscr{W}_{ \pm}$satisfies

$$
X_{ \pm} \mathscr{Z}_{ \pm}+\partial_{\lambda} F_{ \pm}\left(y, \mathscr{W}_{ \pm}\right) \mathscr{Z}_{ \pm}=0 .
$$

Because $\partial_{\lambda} F_{ \pm} \geq 0$, this implies that

$$
\left\{\left(\partial_{t} \pm \partial_{r}\right) \pm(d-1) / r\right\}\left(\mathscr{Z}_{ \pm}\right)^{2} \leq 0,
$$

and integration along characteristics yields

$$
\begin{aligned}
& \left|\partial_{\theta} \mathscr{W}_{+}(t, x, \theta)\right|^{2} \leq\{(r-t) / r\}^{d-1}\left|\partial_{\theta} \mathscr{W}_{0,+}(x-t \hat{x}, \theta)\right|^{2}, \\
& \left|\partial_{\theta} \mathscr{W}_{-}(t, x, \theta)\right|^{2} \leq\{(r+t) / r\}^{d-1}\left|\partial_{\theta} \mathscr{W}_{0,+}(x-t \hat{x}, \theta)\right|^{2} .
\end{aligned}
$$

To justify this calculation, it is sufficient to replace the exact derivative $\partial_{\theta} \mathscr{W}$ by the finite difference $\mathscr{Z}^{(h)}:=\{\mathscr{W}(y, \theta+h)-\mathscr{W}(y, \theta)\} / h$ which satisfies (4.6.34), thanks to the monotonicity of $F$.

Part (i) of the theorem is a direct consequence of estimates (4.6.34) and (4.6.35). Similarly, they imply $L^{\infty}$ bounds for $\partial_{\theta} \mathscr{W}_{ \pm}$as soon as $L^{\infty}$ bounds are given for the data. Because the average of $\mathscr{W}_{ \pm}(y, \cdot)$ is 0 , the $L^{\infty}$ norm of the periodic function $\mathscr{W}_{ \pm}(y, \cdot)$ is dominated by the $L^{1}$ norm of $\partial_{\theta} \mathscr{W}_{ \pm}(y, \cdot)$ and the $L^{\infty}$ estimates for $\mathscr{W}_{ \pm}$follow. This finishes the proof of Proposition 4.6.8.

4.7. Proof of asymptotics. Proposition 4.3.9 and Corollary 4.5.6 show that one has asymptotics with strong profiles. In our context, Lemma 3.2.7 allows asymptotics in $L^{2}$ away from the axis $\{x=0\}$. Next, under Assumption 4.6.7, we prove the asymptotics (2.4.15), (2.4.16), completing the proof of Theorem 2.4.3.

Theorem 4.7.1. Assume condition (2.1.2), and let $u^{\varepsilon}$ denote the family of solutions of (2.1.1) with data satisfying Assumption 2.4.1. Let $\left(\underline{u}, \mathscr{W}_{ \pm}\right)$be the unique solution of (4.6.2) (4.6.6) which satisfies properties (4.4.1), (4.4.3)(4.4.5) and (4.6.10), (4.6.11). Then,

$$
\partial_{t} u^{\varepsilon}(t, x) \sim \partial_{t} \underline{u}(t, x)+\mathscr{W}_{+}\left(t, x, \chi_{+}(t, x) / \varepsilon\right)+\mathscr{W}_{-}\left(t, x, \chi_{-}(t, x) / \varepsilon\right),
$$

$$
\partial_{x_{j}} u^{\varepsilon}(t, x) \sim \partial_{x_{j}} \underline{u}(t, x)+\frac{x_{j}}{|x|}\left\{\mathscr{W}_{-}\left(t, x, \chi_{-}(t, x) / \varepsilon\right)-\mathscr{W}_{+}\left(t, x, \chi_{+}(t, x) / \varepsilon\right)\right\},
$$

in $L^{q}(\omega)$ for all $q<2$ and all bounded $\omega \subset \Omega$, and in $L^{2}(\omega)$ for all $\omega \subset \subset \Omega^{*}$. Proof. (a) One can extract subsequences such that conditions (4.4.2) hold. We prove (4.7.1), (4.7.2) for such subsequences. Uniqueness of solutions of (4.6.2), (4.6.6) implies that these asymptotics hold for the whole family. So, we assume that (4.4.2) is satisfied. Then Corollary 4.5.6 asserts that the variances $\sigma_{ \pm}$of the two-scale Young measures associated to $w_{ \pm}^{\varepsilon}$ vanish. Proposition 4.3.9 implies that

(4.7.3)

$$
w_{+}^{\varepsilon} \sim \mathscr{W}_{+}\left(y, \chi_{+}(y) / \varepsilon\right) \text { in } L^{q}(\omega) \text { for all } q<2 \text { and all bounded } \omega \subset \Omega .
$$


(b) Next we prove that

$$
w_{+}^{\varepsilon} \sim \mathscr{W}_{+}\left(y, \chi_{+}(y) / \varepsilon\right) \text { in } L^{2}(\omega) \text { for all } \omega \subset \subset \Omega^{*} .
$$

Proposition 4.5.3 asserts that $\mathscr{W}_{+} \in L^{2}\left(\Omega_{T} \times \mathbb{T}\right) \cap L^{p}(\Omega \times \mathbb{T})$ and $\Gamma_{k, l} \mathscr{W}_{+} \in$ $L^{2}\left(\Omega_{T} \times \mathbb{T}\right)$. The transport equation (4.5.8) shows that $\left(\partial_{t}+\partial_{r}\right) \mathscr{W}_{+} \in L^{p^{\prime}}(\omega)$, if $\omega \subset \subset \Omega^{*}$. Since $\left(\partial_{t}+\partial_{r}\right) \chi_{+}=\Gamma_{k, l} \chi_{+}=0$, Proposition 4.2 .5 shows that one can choose $m^{\varepsilon} \sim \mathscr{W}_{+}\left(y, \chi_{+}(y) / \varepsilon\right)$ in $L^{2}\left(\Omega_{T}\right) \cap L^{p}(\Omega)$ such that $\Gamma_{k, l} m^{\varepsilon} \sim$ $\left\{\Gamma_{k, l} \mathscr{W}_{+}\right\}\left(y, \chi_{+}(y) / \varepsilon\right)$ in $L^{2}\left(\Omega_{T}\right)$ and $\left(\partial_{t}+\partial_{r}\right) m^{\varepsilon} \sim\left\{\left(\partial_{t}+\partial_{r}\right) \mathscr{W}_{+}\right\}\left(y, \chi_{+}(y) / \varepsilon\right)$ in $L^{p^{\prime}}(\omega)$.

To complete the proof of (4.7.4), it suffices to show that $z^{\varepsilon}:=w_{+}^{\varepsilon}-m^{\varepsilon}$ converges strongly to zero in $L^{2}(\omega)$. The strategy is to apply Lemma 3.2.7 to the families $z^{\varepsilon}$ and $w_{-}^{\varepsilon}$. The estimates on $w_{ \pm}^{\varepsilon}$ and the choice of $m^{\varepsilon}$ show that $z^{\varepsilon}$ and $w_{-}^{\varepsilon}$ are bounded in $L^{2}\left(\Omega_{T}\right)$. Since $w_{+}^{\varepsilon}+w_{-}^{\varepsilon}$ and $m^{\varepsilon}$ are bounded in $L^{p}(\omega)$, it follows that $z^{\varepsilon}+w_{-}^{\varepsilon}$ is bounded in $L^{p}(\omega)$. By definition (3.2.2), $w_{-}^{\varepsilon}$ converges weakly to zero. By part (a), $z^{\varepsilon}$ converges strongly to zero in $L^{q}(\omega)$ for all $q<2$. To apply Lemma 3.2.7, it remains to verify that $z^{\varepsilon} w_{-}^{\varepsilon}$ converges weakly to zero.

(3.2.5) and equations (3.2.4) imply that $\Gamma_{k, l} w_{ \pm}^{\varepsilon}$ is bounded in $L^{2}\left(\Omega_{T}\right)$ and that $\left(\partial_{t} \pm \partial_{r}\right) w_{ \pm}^{\varepsilon}$ is bounded in $L^{p^{\prime}}(\omega)$. By Proposition 4.2.5, $\Gamma_{k, l} m^{\varepsilon}$ is bounded in $L^{2}\left(\Omega_{T}\right)$ and $\left(\partial_{t}+\partial_{r}\right) m^{\varepsilon}$ is bounded in $L^{p^{\prime}}(\omega)$. Therefore $\Gamma_{k, l} z^{\varepsilon}$ is bounded in $L^{2}\left(\Omega_{T}\right)$ and $\left(\partial_{t}+\partial_{r}\right) z^{\varepsilon}$ is bounded in $L^{p^{\prime}}(\omega)$. Hence part (b) of the proof of Theorem 3.2.2 applies to the families $z^{\varepsilon}$ and $w_{-}^{\varepsilon}$ to prove that $z^{\varepsilon} w_{-}^{\varepsilon}$ converges weakly to zero.

(c) Next, use the relations

$$
\begin{gathered}
\partial_{t} u^{\varepsilon}=\partial_{t} \underline{u}+w_{+}^{\varepsilon}+w_{-}^{\varepsilon}, \\
\partial_{x_{j}} u^{\varepsilon}=\partial_{x_{j}} \underline{u}+\frac{x_{j}}{|x|}\left(w_{-}^{\varepsilon}-w_{+}^{\varepsilon}\right)+\sum \frac{x_{k}}{|x|^{2}} \Gamma_{j, k}\left(u^{\varepsilon}-\underline{u}\right) .
\end{gathered}
$$

Property (2.2.2) implies that $\Gamma_{j, k}\left(u^{\varepsilon}-\underline{u}\right)$ converges strongly to zero in $L^{2}(\omega)$ for all bounded $\omega$ and (4.7.3), (4.7.4) immediately imply (4.7.1), (4.7.2).

Remark 4.7.2. So far, we have used little smoothness for the profiles $\mathscr{W}_{0, \pm}$. We have only used that they belong to $L^{2}\left(\mathbb{R}^{d} \times \mathbb{T}\right)$, and the weaker form of (4.5.29)

$$
w_{0, \pm}^{\varepsilon}(x) \sim \mathscr{W}_{0, \pm}(x, \chi(|x|) / \varepsilon) \text { in } L_{\text {loc }}^{2}\left(\mathbb{R}^{d}\right) .
$$

Theorem 4.7.3. When Assumption 4.7.5 holds, the asymptotics (4.7.1) and (4.7.2) can be written as

$$
\partial_{x_{j}} u^{\varepsilon}(y)=\partial_{x_{j}} \underline{u}(y)+\frac{x_{j}}{|x|}\left\{\mathscr{W}_{-}\left(y, \chi_{-}(y) / \varepsilon\right)-\mathscr{W}_{+}\left(y, \chi_{+}(y) / \varepsilon\right)\right\}+e_{j}^{\varepsilon},
$$

where the $e_{j}^{\varepsilon}$ converge strongly in $L^{q}(\omega)$ for all $q<2$ and for all bounded $\omega \subset \Omega$, and in $L^{2}(\omega)$ for all compact $\omega \subset \subset \Omega^{*}$.

Proof. Proposition 4.6.8 implies that $\partial_{\theta} \mathscr{W}_{ \pm} \in L^{2}\left(\left[0, T\left[\times \mathbb{R}^{d} \times \mathbb{T}\right)\right)\right.$ for all $T>$ 0 . Part (iii) of Proposition 4.2.3 shows that (4.7.1), (4.7.2) imply (4.7.6), (4.7.7). 


\section{BLOW UP IN NONDISSIPATIVE EQUATIONS}

In the introduction, we gave a formal argument suggesting that focusing may cause blow up and breakdown of existence for nondissipative equations. In this section we illustrate this idea with two examples. The first deals with a genuinely accretive nonlinearity, $F:=-\left(\partial_{t} u\right)^{3}$. The transport equation is of the form (1.6) and explosive. We prove, for this example, that the blow up of solutions to this profile equations corresponds to blow up for the solutions of the original problem (see Remark 5.1.2). In particular, the blow up of the profile equation occurs before the support of the solutions reach the caustics.

The second example is a modification of an example of [JMR 2]. The nonlinearity is $F:=\left(\partial_{t} u\right)^{2}-\left|\nabla_{x} u\right|^{2}$. Then the transport equation which corresponds to (1.6) is linear. Nevertheless blow up and breakdown of existence do occur at the first time of focusing. For this example, we are able to show that the exact solutions $u^{\varepsilon}$ cannot be extended as weak solutions after focusing.

5.1. An example of blow up before focusing. Consider in dimension $d=3$ the semilinear wave equation

$$
\square u^{\varepsilon}-\left(\partial_{t} u\right)^{3}=0
$$

with oscillating Cauchy data

$$
u_{\mid t=0}^{\varepsilon}(x)=\varepsilon \mathscr{U}_{0}(|x|,|x| / \varepsilon), \quad \partial_{t} u_{\mid t=0}^{\varepsilon}(x)=\mathscr{U}_{1}(|x|,|x| / \varepsilon) .
$$

The profiles are assumed to vanish for $|x| \leq 2$. For small times, [JR] gives the behaviour of $\nabla u^{\varepsilon}$. The profile equations are (2.4.7)-(2.4.12), corresponding to the nonlinearity $F(\lambda):=-\lambda^{3}$.

To simplify matters, assume the Cauchy data for $\mathscr{W}_{+}$is 0 , i.e. that

$$
\mathscr{U}_{1}(r, \theta):=\partial_{\theta} \mathscr{U}_{0}(r, \theta) \text {. }
$$

Then $\mathscr{W}_{+}$must be 0 , at least for small times, and the equation for $\mathscr{W}_{-}$simplifies to

$$
2\left(\partial_{t}-\partial_{r}\right) \mathscr{W}_{-}-2 r^{-1} \mathscr{W}_{-}-F_{-}\left(t, r, \mathscr{W}_{-}\right)=0
$$

with

$$
\begin{gathered}
F_{-}(t, r, \lambda):=\lambda^{3}+3 \lambda^{2} \partial_{t} \underline{u}+3 \lambda\left(\partial_{t} \underline{u}\right)^{2}-f(t, r), \\
f(t, r):=\int\left\{\left(\mathscr{W}_{-}(t, r, \theta)\right)^{3}+3\left(\mathscr{W}_{-}(t, r, \theta)\right)^{2} \partial_{t} \underline{u}(t, r)\right\} d \theta .
\end{gathered}
$$

The equation for $\underline{u}$ is

$$
\square \underline{u}-f=0 .
$$

With (5.1.2), (5.1.3), we see that the Cauchy data for $\underline{u}$ vanish, since $\mathscr{U}_{1}=\partial_{\theta} \mathscr{U}_{0}$ has mean value zero. The Cauchy data for $\mathscr{W}_{-}$is $\mathscr{W}_{0}:=\partial_{\theta} \mathscr{U}_{0}(r, \theta)$.

If we further assume that $\mathscr{U}_{0}(r, \theta)$ is even in $r$, then $\mathscr{W}_{0}$ is odd, and the equation

$$
2\left(\partial_{t}-\partial_{r}\right) \mathscr{W}-2 r^{-1} \mathscr{W}-(\mathscr{W})^{3}=0, \quad \mathscr{W}_{\mid t=0}=\mathscr{W}_{0}
$$

has a unique odd solution. Then, $\mathscr{W}^{3}$ is also odd, its average in $\theta$ vanishes, and $(0, \mathscr{W})$ satisfies the profile equations (5.1.7), (5.1.6). By uniqueness, we conclude that $\underline{u}=0$ and that $\mathscr{W}_{-}$is the solution $\mathscr{W}$ of $(5.1 .8)$. 
Introduce

$$
b(r, \theta):=\frac{r \mathscr{W}_{0}(r, \theta)}{\sqrt{1+r \mathscr{W}_{0}^{2}(r, \theta)}}
$$

so that $b^{2}<r$ and

$$
\mathscr{W}_{0}(r, \theta):=\frac{b(r, \theta)}{\sqrt{r\left(r-b^{2}(r, \theta)\right)}} .
$$

Then the solution $\mathscr{W}$ to $(5.1 .8)$ is

$$
\mathscr{W}(t, r, \theta):=\frac{b(t+r, \theta)}{\sqrt{r\left(r-b^{2}(t+r, \theta)\right)}} .
$$

This solution exists as long as $b^{2}(t+r, \theta)<r$. Clearly, there are $b$ 's such that the first time of appearance of points $r$ such that $b^{2}(t+r, \theta)=r$ is smaller than 2, the first time of focusing.

For instance, assume that

$$
b(r, \theta)=\beta(r) \sin \theta
$$

where $\beta$ is supported in $[2,4]$ and satisfies

$$
r-\beta^{2}(r) \geq 1 / 2 \text { on }[2,4], \quad \text { and }, \quad r-\beta^{2}(r)=1 / 2 \text { on }[5 / 2,7 / 2] \text {. }
$$

Then, for $t+r$ in the support of $\beta$ and $t<T^{*}:=1 / 2, r-\beta^{2}(t+r) \geq$ $(1 / 2)-t>0$ and for $t=T^{*}, r-\beta^{2}(t+r)=0$ for $r \in[2,3]$. Thus, $\mathscr{W}$ is well defined on $\Omega:=\left[0,1 / 2\left[\times \mathbb{R}^{3}, \mathscr{W}\right.\right.$ vanishes outside $\{2 \leq t+r \leq 4\}$ and on $\Omega_{o}:=\Omega \cap\{5 / 2 \leq t+r \leq 7 / 2\}$

$$
|\mathscr{W}| \geq c|\sin \theta|\left\{\left(T^{*}-t\right)+\cos ^{2} \theta\right\}^{-1 / 2} \text {. }
$$

This implies that $\mathscr{W}^{3}$ is not integrable on $\Omega_{o} \times \mathbb{T}$ near $t=T^{*}, \theta=\pi / 2$.

Proposition 5.1.1. With $b$ as in (5.1.12), (5.1.13), define $\mathscr{W}_{0}$ by (5.1.10). Let $\mathscr{U}_{1}:=\mathscr{W}_{0}$ and $\mathscr{U}_{0}:=\left(\partial_{\theta}\right)^{-1} \mathscr{W}_{0}$. Then, for any $T<T^{*}$, there is an $\varepsilon(T)>0$, such that the solution $u^{\varepsilon}$ of $(5.1 .1),(5.1 .2)$ exists and is smooth on $[0, T] \times \mathbb{R}^{3}$. However,

$$
\liminf _{\varepsilon \rightarrow 0} \int_{0}^{T} \int\left|\partial_{t} u^{\varepsilon}(t, x)\right|^{3} d t d x \rightarrow+\infty \quad \text { as } T \rightarrow T^{*} \text {. }
$$

Remark 5.1.2. The first statement means that the life span $T(\varepsilon)$ of $u^{\varepsilon}$ as a smooth solution is larger than $T^{*}-\delta$ if $\varepsilon$ is small enough. On the other hand, (5.1.15) means that one cannot expect the global existence of (weak) solutions in $H^{1}$ with $\partial_{t} u^{\varepsilon}$ uniformly bounded in $L^{3}$ for times $\geq T^{*}$. Remark that $u^{\varepsilon}=0$ in $\{t+|x| \leq 2\}$ so that (5.1.15) takes place at a finite distance from $x=0$, that is, well before the rays reach the time axis.

Proof. Following [JR], one constructs a complete asymptotic solution

$$
u_{a}^{\varepsilon} \sim \sum_{k=0}^{\infty} \varepsilon^{j+1} \mathscr{U}_{j}(t, r,(t+r) / \varepsilon,(r-t) / \varepsilon)
$$

whose leading term $\mathscr{U}_{1}$ only depends on the phase $(t+r) / \varepsilon$, and is related to the solution $\mathscr{W}$ of $(5.1 .8)$ through the simple relation, $\partial_{\theta} \mathscr{U}_{1}=\mathscr{W}$. The other 
profiles are determined by linear equations, and therefore are smooth and well defined on $\Omega=\left[0, T^{*}\left[\times \mathbb{R}^{3}\right.\right.$.

For any $T<T^{*}$, if $\varepsilon$ is small enough, (5.1.16) defines a smooth approximate solution of the equations, with norms bounded uniformly in $\varepsilon$. Then one can prove, for $\varepsilon$ small enough, the existence on $[0, T]$ of a smooth exact solution $u^{\varepsilon}$, such that $u^{\varepsilon}-u_{a}^{\varepsilon} \rightarrow 0$ and $\partial_{t} u^{\varepsilon}-\partial_{t} u_{a}^{\varepsilon} \rightarrow 0$ in $L^{\infty}\left([0, T] \times \mathbb{R}^{3}\right)$ as $\varepsilon \rightarrow 0$ (see [JR]). This shows the existence part in Proposition 5.1.1.

Moreover, this also implies that

$$
\partial_{t} u^{\varepsilon}(t, x)-\mathscr{W}(t, r,(t+r) / \varepsilon) \rightarrow 0 \quad \text { in } L^{\infty} \text { as } \varepsilon \rightarrow 0 .
$$

Lemma 4.1 .3 and (5.1.14) imply that the $L^{3}$ norm of $\mathscr{W}(t, r,(t+r) / \varepsilon)$ on $[0, T] \times \mathbb{R}^{3}$ tends to $+\infty$ as $T$ tends to $T^{*}$. With $(5.1 .17)$, this implies $(5.1 .15)$ and the proposition is proved.

5.2. An example from [JMR 2]. In $\mathbb{R}^{1+5}$ consider the semilinear wave equation

$$
\left\{\begin{array}{l}
\square v^{\varepsilon}=\left(\partial_{t} v^{\varepsilon}\right)^{2}-\left|\nabla_{x} v^{\varepsilon}\right|^{2}, \\
v_{\mid t=0}^{\varepsilon}(x)=0, \quad \partial_{t} v_{\mid t=0}^{\varepsilon}(x)=-h^{\varepsilon}(x) .
\end{array}\right.
$$

Let

$$
h^{\varepsilon}(x):=a(|x|) \sin (|x| / \varepsilon)+\varepsilon b(|x|) \cos (|x| / \varepsilon),
$$

with $a$ smooth, supported in [1,3], $a>0$ in ]1,3[, and $a^{\prime}>0, a^{\prime \prime}>0$ in ]1, 2[. We make a special choice of $b$, to simplify the argument,

$$
b(r):=a^{\prime}(r)+3 r^{-1} a(r) .
$$

If $v^{\varepsilon}$ is a Lipschitz solution, then $u^{\varepsilon}=1-e^{v^{\varepsilon}}$ satisfies

$$
\left\{\begin{array}{l}
\square u^{\varepsilon}=0, \\
u_{\mid t=0}^{\varepsilon}(x)=0, \quad \partial_{t} u_{\mid t=0}^{\varepsilon}(x)=h^{\varepsilon}(x) .
\end{array}\right.
$$

Conversely, let $u^{\varepsilon}$ be the unique solution of (5.2.4). Then $u^{\varepsilon}$ is smooth and $u^{\varepsilon}<1$ for $t$ small. Let $\Omega_{\varepsilon}$ be the connected open subset of $\left\{u^{\varepsilon}<1\right\}$ which contains this initial strip. Then $v^{\varepsilon}:=\operatorname{Ln}\left(1-u^{\varepsilon}\right)$ satisfies (5.2.1) on $\Omega_{\varepsilon}$. The right-hand side $F^{\varepsilon}:=\left(\partial_{t} v^{\varepsilon}\right)^{2}-\left|\nabla_{x} v^{\varepsilon}\right|^{2}$ in $(5.2 .1)$ is

$$
F^{\varepsilon}=\left\{\left(\partial_{t} u^{\varepsilon}\right)^{2}-\left|\nabla_{x} u^{\varepsilon}\right|^{2}\right\} /\left(1-u^{\varepsilon}\right)^{2} .
$$

By finite speed of propagation, $u^{\varepsilon}=0$ in the cone $\bar{C}:=\{0 \leq t+|x| \leq 1\}$, and thus $\Omega_{\varepsilon}$ contains a neighborhood of $\bar{C}$.

Proposition 5.2.1. Let $B_{\rho}$ denote the ball of radius $\rho>0$ centered at $t=1$, $x=0$. Then, for any $\rho>0$, there is an $\varepsilon \in] 0, \rho\left[\right.$ such that the ball $B_{\rho}$ is not contained in $\Omega_{\varepsilon}$ and the solution $v^{\varepsilon}$ on $\Omega_{\varepsilon}$ is such that the interaction term $F^{\varepsilon}:=\left(\partial_{t} v^{\varepsilon}\right)^{2}-\left|\nabla_{x} v^{\varepsilon}\right|^{2}$ is not integrable on $\Omega_{\varepsilon} \cap B_{\rho}$.

Comment 5.2.2. That $F^{\varepsilon}$ is not integrable on $\Omega_{\varepsilon} \cap B_{\rho}$ implies that $v^{\varepsilon}$ cannot be extended as a weak solution of (5.2.1) on $B_{\rho}$. Thus there is no open domain $\Omega$ which contains $\bar{C}$ such that $(5.2 .1)$ has a weak solution on $\Omega$, for all $\varepsilon$.

Remark 5.2.3. For equation (5.2.1), the profile equation which correspond to (1.6) is linear. This can be seen directly, or better from the relation $v^{\varepsilon}:=$ $\operatorname{Ln}\left(1-u^{\varepsilon}\right)$ which for simple waves of the form (1.5) extends to the profiles as $\varepsilon \mathscr{V}=\operatorname{Ln}(1-\varepsilon \mathscr{U}) \sim-\varepsilon \mathscr{U}$. 
Proof of Proposition 5.2.1. Using the explicit fundamental solution of $\square$ in dimension $d=5$ yields

$$
u^{\varepsilon}(t, x)=\int_{S^{4}}\left\{t h^{\varepsilon}(x+t \omega)+\frac{1}{3} t^{2} \omega \cdot \nabla_{x} h^{\varepsilon}(x+t \omega)\right\} d \omega
$$

where $d \omega$ denotes the rotationally invariant measure on $S^{4}$ of total mass 1 . With (5.2.3), one gets

$$
u^{\varepsilon}(t, 0)=(\alpha(t) / \varepsilon+\varepsilon \beta(t)) \cos (t / \varepsilon)
$$

with $\alpha(t):=t^{2} a(t) / 3, \beta(t)=t b(r)+t^{2} b^{\prime}(r) / 3$.

Our assumptions on $a$ imply that $\alpha$ and $\beta$ are increasing functions on [1,2], and for $\varepsilon$ small enough, there is a unique $\left.\left.\tau_{\varepsilon} \in\right] 1,2\right]$ such that

$$
\alpha(t)+\varepsilon^{2} \beta(t)<\varepsilon \text { for } t<\tau_{\varepsilon} \text { and } \alpha\left(\tau_{\varepsilon}\right)+\varepsilon^{2} \beta\left(\tau_{\varepsilon}\right)=\varepsilon .
$$

Moreover, $\tau_{\varepsilon} \rightarrow 1$ as $\varepsilon \rightarrow 0$. Thus $\tau_{\varepsilon} / \varepsilon \rightarrow+\infty$ as $\varepsilon \rightarrow 0$, and one can select a sequence of $\varepsilon$ such that

$$
\tau_{\varepsilon} / \varepsilon=2 \pi n, \quad n \in \mathbb{N} \text { large. }
$$

Restricting $\varepsilon$ to such a sequence, we see that $u^{\varepsilon}(t, 0)<1$ for $t<\tau_{\varepsilon}$ and $u^{\varepsilon}\left(\tau_{\varepsilon}, 0\right)=1$. Moreover, condition (5.2.9) implies that $\partial_{t} u^{\varepsilon}\left(\tau_{\varepsilon}, 0\right)=\alpha^{\prime}\left(\tau_{\varepsilon}\right) / \varepsilon+$ $\varepsilon \beta^{\prime}\left(\tau_{\varepsilon}\right)>0$. On the other hand, $u^{\varepsilon}$ is rotationally invariant and smooth, so that $\nabla_{x} u(t, 0)=0$. Thus, we also have that $\left(\partial_{t} u^{\varepsilon}\left(\tau_{\varepsilon}, 0\right)\right)^{2}-\left|\nabla_{x} u^{\varepsilon}\left(\tau_{\varepsilon}, 0\right)\right|^{2}>0$.

Let $\rho>0$ be given. Choose $\varepsilon \in] 0, \rho$ [ so small that $\tau_{\varepsilon}<1+\rho / 4$, with (5.2.9) satisfied. Since $\left(\tau_{\varepsilon}, 0\right) \notin \Omega_{\varepsilon}$, it follows that $B_{\rho}$ is not contained in $\Omega_{\varepsilon}$.

Since $u^{\varepsilon}(t, 0)<1$ for $t<\tau_{\varepsilon}, u^{\varepsilon}\left(\tau_{\varepsilon}, 0\right)=1$ and $\partial_{t} u^{\varepsilon}\left(\tau_{\varepsilon}, 0\right)>0$, there is a $\delta>0$ such that, for all $x$ with $|x| \leq \delta$, there is a unique $\left.\left.\tau_{\varepsilon}(x) \in\right] 1, \rho / 2\right]$ such that

$$
u^{\varepsilon}(t, x)<1 \text { for } t<\tau_{\varepsilon}(x) \text { and } u^{\varepsilon}\left(\tau_{\varepsilon}(x), x\right)=1,
$$

$$
\partial_{t} u^{\varepsilon}\left(\tau_{\varepsilon}(x), x\right)>0 \text { and }\left(\partial_{t} u^{\varepsilon}\left(\tau_{\varepsilon}(x), x\right)\right)^{2}-\left|\nabla_{x} u^{\varepsilon}\left(\tau_{\varepsilon}(x), x\right)\right|^{2}>0
$$

Property (5.2.10) means that

$$
\Omega_{\varepsilon} \cap\{|x| \leq \delta\}=\left\{(t, x)|| x \mid \leq \delta \text { and } 0<t<\tau_{\varepsilon}(x)\right\} .
$$

Furthermore, (5.2.11) implies that for $|x|<\delta$ and $t<\tau_{\varepsilon}(x)$ one has

$$
F^{\varepsilon} \geq c\left(\tau_{\varepsilon}(x)-t\right)^{-2} \text {. }
$$

Thus $F^{\varepsilon}$ is nonintegrable on $\Omega_{\varepsilon} \cap\{|x| \leq \delta\}=\{(t, x)|| x \mid \leq \delta$ and $0<t<$ $\left.\tau_{\varepsilon}(x)\right\}$.

This finishes the proof of Proposition 5.2.1.

\section{REFERENCES}

[D] J. M. Delort, Oscillations semi-linéaires multiphasées compatibles en dimension deux ou trois d'espace, Comm. Partial Differential Equations 16 (1991), 845-872.

[DP1] R. J. Di Perna, Compensated compactness and general systems of conservation laws, Trans. Amer. Math. Soc. 292 (1985), 383-420.

[DP2] - Measure valued solutions of conservation laws, Arch. Rational Mech. Anal. 8 (1985), 223-270. 
[DP-M] R. J. Di Perna and A. Majda, Oscillations and concentration in weak solutions of the incompressible fluid equations, Comm. Math. Phys. 108 (1987), 667-689.

[E] W. E, Homogeneization of linear and nonlinear transport equations, Comm. Pure Appl. Math. 45 (1992), 301-326.

[ES] W. E and D. Serre, Correctors for the homogenization of conservation laws with oscillatory forcing terms, preprint, 1992.

[Ev] L. C. Evans, Weak convergence methods for non linear partial differential equations, Amer. Math. Soc., Providence RI, 1990.

[G1] O. Guès, Développements asymptotiques de solutions exactes de systèmes hyperboliques quasilinéaires, Asymptotic Anal. 6 (1993), 241-270.

[G2] - Ondes multidimensionnelles E-statifiées et oscillations, Duke Math. J. 68 (1992), 401-446.

[JMR1] J.-L. Joly, G. Metivier, and J. Rauch, Remarques sur l'optique géométrique non linéaire multidimensionelle, Séminaire Equations aux Dérivées Partielles de l'Ecole Polytechnique 1990-1991, Exposé no. 1.

[JMR2] _ Coherent and focusing multidimensional nonlinear geometric optics, Ann. Sci. École Norm. Sup. 28 (1995), 51-113.

[JMR3] _ Generic rigourous asymptotic expansions for weakly nonlinear multidimensional oscillatory waves, Duke Math. J. 70 (1993), 373-404.

[JMR4] __ Coherent nonlinear waves and the Wiener algebra, Ann. Inst. Fourier (Grenoble) 44 (1994), 167-196.

[JMR5] _ Nonlinear oscillations beyond caustics, preprint.

[JR] J-L. Joly and J. Rauch, Justification of multidimensional single phase semilinear geometric optics, Trans. Amer. Math. Soc. 330 (1992), 599-625.

[L] J. L. Lions, Quelques méthodes de résolution de problèmes aux limites non linéaires, Dunod and Gauthiers-Villars, Paris, 1969.

[LS] J. L. Lions and W. Strauss, Some nonlinear evolution equations, Bull. Soc. Math. France 93 (1965), 43-96.

[N] G. Nguetseng, A general convergence result for a functional related to the theory of homogeneisation, SIAM J. Math. Anal. 20 (1989), 608-623.

[RR1] J. Rauch and M. Reed, Striated solutions of semilinear two speed wave equations, Indiana Univ. Math. J. 34 (1985), 337-353.

[RR2] - Nonlinear superposition and absorption of delta waves in one space dimension, $\mathrm{J}$. Funct. Anal. 73 (1987), 152-178.

[S] S. Schochet, Fast singular limit of hyperbolic PDEs, J. Differential Equations 114 (1994), 476-512.

[T] L. Tartar, Compensated compactness and applications to partial differential equations, Research Notes in Mathematics, Nonlinear Analysis and Mechanics, Heriot-Watt Symposium, vol. 4 (R. J. Knops, ed.), Pitman Press, New York, 1979.

[Y] L. C. Young, Lectures on the calculus of variations and optimal control theory, Saunders, Philadelphia and London, 1969.

Département de Mathématiques, Université de Bordeaux I, CNRS UA 226, BordeauX, FRANCE

E-mail address: joly@math.u-bordeaux.fr

Département de Mathématiques, Université de Rennes I, CNRS UA 305, Rennes, FRANCE

E-mail address: metivier@univ-rennes1.fr

Department of Mathematics, The University of Michigan, Ann Arbor, Michigan 48109

E-mail address: rauch@math.lsa.umich.edu 